Programa de Doctorado de Inteligencia Artificial Escuela Técnica Superior de Ingenieros Informáticos

PhD Thesis

\title{
Autonomous Management of Internet of Things Technologies
}

\author{
Author: Alvaro Brandon \\ Supervisors: Prof. Dra. María S. Pérez Hernández \\ Dr. Marc Solé
}

June, 2018 
Tribunal nombrado por el Sr. Rector Magfco. de la Universidad Politécnica de Madrid, el día 11 de Junio de 2018.

Presidente: Dr. Presidente Presidente Presidente

Vocal: Dr. Vocal Vocal Vocal

Vocal: $\quad$ Dr. Vocal2 Vocal2 Vocal2

Vocal: $\quad$ Dr. Vocal3 Vocal3 Vocal3

Secretario: Dr. Secretario Secretario Secretario

Suplente: Dr. Suplente Suplente Suplente

Suplente: Dr. Suplente2 Suplente2 Suplente2

Realizado el acto de defensa y lectura de la Tesis el día XX de MMMMM de YYYY en la Escuela Técnica Superior de Ingenieros Informáticos

Calificación:

EL PRESIDENTE

VOCAL 1

VOCAL 2

VOCAL 3

EL SECRETARIO 
TBD

TBD 
Agradecimientos

TBD 


\begin{abstract}
The overwhelming amount of data that needs to be processed nowadays has driven the design of increasingly complex distributed systems. This complexity is further exacerbated by new decentralised approaches, which process the data near where it is generated, such as Fog or Edge computing. Having full control of these infrastructures becomes a challenge even for the most experienced administrators, as there are many heterogeneous technologies and factors involved. Usually, administrators follow a process that involves using monitoring tools and browsing through logs in order to find insights that explain events happening in the system. Instead, this cumbersome process can be partially or totally automatised through the use of artificial intelligence techniques (AI) that extract these insights from all the incoming monitored information. In this thesis we propose a series of AI models that are able to solve some of the common problems that administrators find in these kind of systems. Namely, we focus on providing observability for a Fog computing infrastructure, optimising the task parallelisation of Big Data jobs and performing root cause analysis for microservice architectures. Through a series of experiments, we demonstrate how the AI models can be used to increase the performance and reliability of data analytics jobs and the new technologies that power them.
\end{abstract}




\section{Resumen}

La ingente cantidad de datos que necesitan ser procesados hoy en día ha impulsado el diseño de sistemas distribuidos cada vez más complejos. Esta complejidad se acrecenta aún más con el uso de nuevos modelos descentralizados, en el que los datos son procesados cerca de su origen, con Fog y Edge computing como algunos ejemplos. Conseguir el control total de estas infraestructuras es todo un reto, incluso para administradores de sistema expertos, debido a las dispares tecnologías y factores involucrados. Normalmente, los administradores siguen un proceso en el que analizan los datos provenientes de logs o herramientas de monitorización para extraer conocimiento que pueda explicar la ocurrencia de ciertos eventos. Este engorroso proceso puede ser automatizado parcialmente o en su totalidad mediante el uso de la inteligencia artificial (IA), extrayendo dicho conocimiento a través de la información monitorizada. En esta tesis proponemos una serie de modelos de IA, cuyo objetivo es resolver algunos de los problemas más comunes que se encuentran los administradores en este tipo de sistemas. Principalmente, nos centramos en dar visibilidad del estado de una infraestructura Fog, en optimizar la paralelización de trabajos Big Data y en diagnosticar la causa raíz de un problema en una arquitectura de microservicios. A través de una serie de experimentos, somos capaces de demostrar cómo esta inteligencia generada se puede utilizar para incrementar el rendimiento y la fiabilidad de los trabajos para análisis de datos y las nuevas tecnologías que los soportan. 


\section{Contents}

I Introduction: Motivation and Hypothesis 1

1 Introduction 3

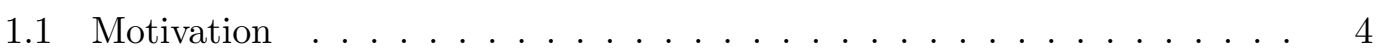

1.2 Objectives and hypothesis $\ldots \ldots \ldots \ldots \ldots$

1.3 Contributions ........................ 6

1.4 Organization of the manuscript . . . . . . . . . . 8

II Context: The IoT technological stack and its management 11

2 The Internet of Things and its technological stack 13

2.1 IoT: Definition and technologies used . . . . . . . . . . . 14

2.2 Enabling technologies for the Internet of Things . . . . . . . . . . . 18

2.3 Conclusions: Three key technologies for IoT . . . . . . . . . . . . . 29

3 Autonomic Management of the IoT technologies 31

3.1 Autonomic Computing . . . . . . . . . . . . . . . 31

3.2 Monitoring as a key element of autonomic computing . . . . . . . . . 39

3.3 Conclusion: autonomic computing facilitates the management of infrastructures . . . . . . . . . . . . . . . . . . 43

III Contributions: Managing, optimising and monitoring the IoT technological stack 45

4 General Methodology 47

4.1 Artificial Intelligence for autonomic computing . . . . . . . . . . . 47 
4.2 Conclusions: creating intelligence by monitoring the system . . . . . . 52

5 FMonE: An effective monitoring tool at the edge 55

5.1 Motivating scenarios . . . . . . . . . . . . . . . 56 56

5.2 Requirements of a Fog monitoring system . . . . . . . . . . . 58

5.3 FMonE design: Tackling the challenges . . . . . . . . . . . . 61 61

5.4 Evaluation . . . . . . . . . . . . . . . . . 68 68

5.5 Conclusions: Fog observability is possible and containers are a key technology in IoT . . . . . . . . . . . . . . . . . . 80

6 Root cause analysis for microservice architectures 83

6.1 Graph Based RCA . . . . . . . . . . . . . . . . . . . 84

6.2 Graph similarity engine . . . . . . . . . . . . . . . . . . 91

6.3 Monitoring and building the graphs . . . . . . . . . . . 96

6.4 Evaluation of the graph-based approach . . . . . . . . . . . 99

6.5 Transferring knowledge through log analysis and metrics distribution . . 111

6.6 Conclusions: graphs are a suitable model for microservices . . . . . . . . 117

7 Optimising task parallelisation in Big Data analytics 121

7.1 Task parallelisation with Spark and YARN . . . . . . . . . . . 122

7.2 Studying the impact of parallelism . . . . . . . . . . . . . . . 123

7.3 Model to find the optimal level of parallelism . . . . . . . . . . . . 127

7.4 Evaluation . . . . . . . . . . . . . . . . . . . . . . . . . 142

7.5 Conclusions: controlling and optimising big data workloads . . . . . . 152

IV Conclusions: Achievements and Perspectives 155

8 Conclusions $\quad 157$

8.1 Achievements . . . . . . . . . . . . . . . . . . . 159

8.2 Tools and artifacts for reproducibility $\ldots \ldots \ldots \ldots$

8.3 Future Work . . . . . . . . . . . . . . . . . . . . . . . . 161

Bibliography 


\section{List of Figures}

2.1 Integration of the things and the Cloud $(81) \ldots \ldots \ldots \ldots$

2.2 The network stack for IoT devices $1 \ldots \ldots \ldots$

2.3 A simplification of the Fog architecture $(90) \ldots \ldots \ldots$

2.4 Comparing containers to virtual machines 2 . . . . . . . . . . 21

2.5 Context and challenges related to IoT enabling technologies . . . . . 30

3.1 The MAPE-K model for autonomic control together with our contribu-

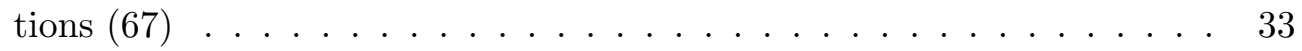

4.1 The data science pipeline used to build AI models for infrastructure management ...................... 49

4.2 Human in the loop ${ }^{3} \ldots \ldots \ldots \ldots \ldots \ldots$

4.3 The framework used to manage and provide observability of the infrastructure. Our contributions are detailed in Chapter 5 for the monitoring part and in Chapter 6 and 7 for the intelligence part of the system . . .

5.1 A Fog infrastructure starts at the edge of the network, where the data is generated at the IoT layer. Small data centers provide computation and storage close to the applications. If data needs to be sent to the central cloud or intermediate gateways, it is aggregated in a bottom-up fashion. 
5.2 The general architecture that supports FMonE. The user interacts with the FMonE framework (through an UI or command line not yet implemented) which acts as a translator between the user needs and a Marathon deployment plan. When the user has built the monitoring workflow this is translated into an FMonE pipeline, which in turn is passed as a JSON file to the Marathon API. Marathon is then responsible for launching the monitoring agents and the back-ends throughout the infrastructure . . . . . . . . . . . . . . . . . 62

5.3 The DCOS architecturet . . . . . . . . . . . . . . . 65 65

5.4 An example of an FMonE pipeline that takes care of three different regions using two back-ends. Users can query the metrics stored in any of the different back-ends. . . . . . . . . . . . . . . . . . . 66 66

5.5 The different concepts that form a monitoring pipeline. A pipeline takes care of one or more regions of the Fog infrastructure. Additionally it may have several back-ends. A set of one or more FMonE agents is launched in each region. These agents have three components to gather the metrics: InPlugin to collect data, Midplugin to process it and OutPlugin to

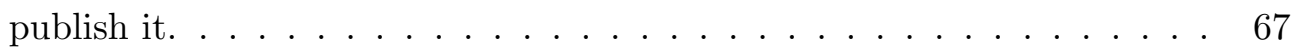

5.6 Several Docker containers are involved in an FMonE pipeline. To extract the metrics, the Docker image for FMonE is started in each host, with docker as the Inplugin parameter, inout for the MidPlugin and rabbitmq as the Outplugin. The RabbitMQ container will also be created as part of the pipeline and its hostname used as a parameter for the previous outplugin. The aggregation will be performed by a different FMonE agent, which will pull the metrics with its corresponding rabbitmq Inplugin, average them with the average Midplugin and store them in a database with the mongodb Outplugin. The pipeline will be completed with the creation of a containerized MongoDB back-end. Marathon and Mesos are used to start these containers in the desired regions . . . . . . 68 
5.7 The configuration of our experiments. A central region hosts both a cluster of Cassandra and Kafka. On the edge, three regions of different size, host the YCSB clients communicating with the Cassandra database together with the FMonE agents sending their metrics to Kafka. Note that Kafka and Cassandra are running on different nodes to avoid overhead. Bandwidth and latency between regions are limited to $4 \mathrm{Mb} / \mathrm{s}$ and $50 \mathrm{~ms}$ respectively. . . . . . . . . . . . . . . . . .

5.8 Impact on the performance of edge applications with different monitoring methods. A typical centralized approach, where all the metrics from the edge are sent to a central location, is compared to two FMonE pipelines that aggregate and store metrics in their own region, respectively. The traffic caused by the monitoring information consumes much of the limited bandwidth at the edge, affecting the number of ops/sec. .

5.9 Overhead of a FMonE deployment. Even when monitoring 15 containers per host, the overhead is negligible for an FMonE pipeline. . . . . . . 76

5.10 Time series of the resource usage for Prometheus and two of the available FMonE back-ends: Kafka and MongoDB. Prometheus is a more resource intensive back-end compared to the other two. Note that the resource usage is important when placing the back-end for metrics on devices with limited resources such as Fog. . . . . . . . . . . . . . .

5.11 Time that it takes to start up the monitoring agents. We measure it when the container image needs to and does not need to be pulled from the Docker repository. We also vary the number of host and containers from 1 host and 1 agent to 15 hosts and 30 agents. Pulling creates an obvious traffic overhead compared to the fast deployment of a container that has already been pulled. . . . . . . . . . . . . . . .

6.1 Simplified example of a multi-attributed graph model for a microservice architecture. An anomaly is generated in the HA Proxy instance due to the unbalanced number of requests that the WordPress instances are receiving . . . . . . . . . . . . . . . . . . 85

6.2 Architecture of the proposed system $\ldots \ldots \ldots \ldots$ 
6.3 An example of a taxonomy explaining a hierarchy of concepts. When comparing graphs, the taxonomy will allow us to reason those concepts situated close to each other in the tree, concluding that they are more similar. For example a DB master is very similar to a load balancer, since they both are coordinators that distribute some load to their slaves. 87

6.4 An illustration of the method that builds the anomalous regions. The anomalous node with the cross shape, generates an anomalous region including neighbours that are located at 1 or 2 hops from anomalous nodes, representing a cascading effect where the performance of one element depends on the others . . . . . . . . . . . . . . 899

6.5 A representation of the mapping between nodes and the similarity score given by the graph similarity function . . . . . . . . . . . . . . 90

6.6 Effect of weights in the matching algorithm. Giving a bigger weight to the shape attribute of the nodes changes the matching in comparison to Figure 6.5. In this case the structure of the graph is not as important as matching nodes with the same shape. . . . . . . . . . . . . . 95

6.7 An example on how we group the different TCP requests traced by sysdig. Each group is going to have four entries for each client ip $\rightarrow$ server ip TCP pipe that will be added as attributes of the edges . . . . . . . 99

6.8 A simple representation of the loadbalancer scenario . . . . . . . . . 100

6.9 A simple representation of the Kafka scenario . . . . . . . . . . 100

6.10 A simple representation of the HDFS + Spark scenario . . . . . . . . . 101

6.11 Design of the experiments to evaluate the RCA framework precision. There are two main steps. First, we process the first and second execution of each scenario to build its system graphs, generate its anomalous region and then, for each anomaly, generate a representative library of patterns for that problem. All of these representative libraries will be combined into one that will be used to establish the RCA of the anomalies induced in the third execution, based on the previously generated patterns. . . . . . . . . . . . . . . . . . 105

6.12 Number of patterns generated for the first, second, third and fourth execution of the training process $\ldots \ldots \ldots \ldots$ 
6.13 The effect of changing the matching threshold in the system. Precision is depicted as bars and the number of patterns generated as a line. . . . 109

6.14 Precision of the graph-based approach compared to a machine learning based approach . . . . . . . . . . . . . . . . 111

6.15 Execution time of the comparison between two graphs with different number of nodes. . . . . . . . . . . . . . . . . . . . . 112

6.16 Scheme presenting context and event window of logs. Both windows start on a first Error or Warning message. . . . . . . . . . . . . . . 114

6.17 An example process of transformation $\log$ entries to vectors. . . . . . . 115

6.18 The Cassandra architecture . . . . . . . . . . . . . . . . . 116

$6.19 \mathrm{~F} 1$ scores for the cross-system diagnostics system. . . . . . . . . . . 118

7.1 Example DAG for WordCount . . . . . . . . . . . . . . . . . 124

7.2 Run time in seconds and power consumption in watts for a kMeans App. The default, best and worst configurations are depicted . . . . . . 126

7.3 Run time in seconds and power consumption in watts for a Page Rank App. The default, best and worst configurations are depicted . . . . . . 127

7.4 A set of waves spanned by two executors with three spark.executor.cores. If we have 16 tasks to be executed then it will take three waves to run them in the cluster . . . . . . . . . . . . . . . . . . . . . 131

7.5 Slim bottom-up aggregation. If we have the metrics for the tasks, Slim can aggregate the ones belonging to a stage to calculate that stage metrics. It does the same at the job and application execution level . . . . . 132

7.6 Mean application metrics values for a stage of a Sort application with different filesizes. We can see how the mean of the metrics is almost the same for 40,81 and 128 tasks . . . . . . . . . . . . . 133

7.7 Changes in the metrics for a stage of a support vector machine implementation in Spark. Note how the CPU metrics change depending on the number of tasks per node. Also garbage collection and the deserialize time of an stage increases with this number. . . . . . . . . . . . . 135 
7.8 $10 \mathrm{CV}$ with $3 \mathrm{~K}$-Fold MAE score for different regression algorithms. GradientBoostingRegressor is the clear winner with a median absolute error of 23 seconds, a maximum of 94 seconds in the last 10th iteration and a minimum of 2.7 seconds for the 3 rd iteration . . . . . . . . 137

7.9 An example with a Sort App, where we iterate through a configuration list. Notice the different parallelism features: $\left\{p_{1}, \ldots, p_{n}\right\}_{r e f}$ represent the parallelism conditions under which the metrics were collected, $\left\{p_{1}, \ldots, p_{n}\right\}_{\text {run }}$ the parallelism conditions under which the application run for that execution and $\left\{p_{1}, \ldots, p_{n}\right\}_{\text {new }}$ the new parallelism conditions that we want to try and the ones we will pass to the machine learning module. The rest of the metrics are kept constant. Then we just have to group by configuration and sum up each of the stages predictions to know which one is best . . . . . . . . . . . . . . . . . . . . 139

7.10 A deadlock situation in which the same configuration is recommended continuously. The application crashes with the default configuration. Based on whichever stages executed successfully the next recommendation also crashes. If we do not get past the crashing point the recommendation will always be the same . . . . . . . . . . . . . 141

7.11 Overhead introduced by monitoring and storing the traces versus nonmonitoring. As we can see the latency is minimum . . . . . . . . . . 146

7.12 Duration of training the model depending on the number of data points. For the whole dataset of 85664 points the duration is 1536 seconds. . . . 147

7.13 Latency in the predictions for different applications. The latency is always less than 1 second with a maximum of 0.519 for a Logistic Regression App . . . . . . . . . . . . . . . . . . . . 147

7.14 Predictions for stages of different apps with the highest duration and so highest impact inside their applications. For Shortest Path the predictions are pretty accurate. For SVM and Logistic Regression the trend of the predicted values follows the real ones. . . . . . . . . . . . . . . 148

7.15 Executions for the non-graph processing applications of the test set. . . 149

7.16 Executions for the graph processing applications of the test set. . . . . 150

7.17 Evolution for the predictions of a recurrently executed shortest path application. . . . . . . . . . . . . . . . . 151 
7.18 Time needed to calculate a recommended configuration with the boosted regression method and the kNeighbours method for each of the applications in our simulated scenario. Note that some of the applications do not use the kNeighbours method, since it is needed only when the application crashes with the default configuration of $1 \mathrm{~g}$ and 1 core . . . 152

7.19 Partial dependence for different applications. Shortest Paths works better with a low number of tasks per node. kMeans best setting is 12 tasks per node. For PCA the points between 0 and 5 corresponds to stages with only one or few tasks, while with many tasks 7 is a good configuration.153 


\section{List of Tables}

5.1 The different YCSB workloads . . . . . . . . . . . . 73

5.2 Resilience of the system . . . . . . . . . . . . . . . 78

5.3 Cloud and Fog monitoring properties. . . . . . . . . . . 79

6.1 Notation table . . . . . . . . . . . . . . . . . . 94

6.2 Confusion matrix for the graph based RCA method . . . . . . . . 106

6.3 Confusion matrix for the boosted decision trees RCA method . . . . . . 110

7.1 Combinations of Spark parameters used for the experiments . . . . . . 125

7.2 Different applications used. File size denotes the three different sizes used for that application. Group by doesn't read any file but creates all the data directly in memory with 100 partitions . . . . . . . . . . 145

8.1 The different steps used in our decision making workflow (Figure 4.1) and how they were used for the two technologies . . . . . . . . 158 


\section{List of Algorithms}

1 Building the training dataset for the machine learning algorithm . . . 136

2 Recommending a parallelism configuration for an application and its input datasize . . . . . . . . . . . . . . . . . . . . 140

3 Finding a configuration for an application that failed . . . . . . . . 142

4 Providing an optimal configuration for a application and a given input file size . . . . . . . . . . . . . . . . . . . . . . . 143 


\section{Part I}

\section{Introduction: Motivation and Hypothesis}





\section{Chapter 1}

\section{Introduction}

The digitalization of many parts of our society has radically changed the world we live in. This revolution is fueled by data that is generated from different sources, enabling data-driven decisions that have been proved to make advancements in several fields, like computer vision or recommendation systems. The amount of data generated is constantly growing and its processing and storage requirements are everyday more demanding. Currently, these requirements can only be fulfilled through the use of distributed systems, such as Cloud or HPC, in which many computers cooperate to execute a program.

One of the driving forces behind this data deluge is the Internet of Things (IoT), which advocates for the connection of everyday objects and cheap sensors to the Internet, enabling the modelling of our world through the data they generate. IoT uses various technologies to achieve this, such as the Cloud and Big Data analytic platforms, which are responsible for processing all the information. However, moving all the data from the devices and sensors to a centralised location such as the Cloud, becomes a problem, due to the communication latency between them and the necessity of making decisions based on the data in almost real-time. Decentralised computing architectures have been brought again to the forefront to solve these issues.

One of these types of architectures is Edge computing, based on the key idea of performing the data processing closer to its source. In this line of thought, Fog computing was designed as a new architecture to complement the Cloud. In Fog computing the Cloud is still present, but is used to centralise decisions that require an aggregated view of the geo-distributed IoT deployment, or for more computation-intensive tasks, 
like training artificial intelligence (AI) models with all the generated data. The intermediary nodes that comprise the Fog stand between the Cloud and the Edge and they filter and pre-process the data when needed, reducing traffic bottlenecks and response times. Some examples of these types of nodes are mobile phones, gateways or cheap computers such as Raspberry Pi's, following a paradigm in which there are many geo-distributed, low computing power devices, instead of a few centralised and powerful servers. The Fog nodes show several particularities, such as having heterogeneous architectures, constrained resources or the possibility of losing their connection capabilities, as many of them are mobile. This hinders the installation and execution process of decentralised applications on top of them, favoring the use of container technologies such as Docker to deploy services across the Fog. Furthermore, containers are a key element in a variant of the service oriented architecture know as microservice architecture. It allows to divide a complex application into smaller parts, which has clear synergies with an infrastructure such as the Fog, where we have a pool of distributed computing resources.

\subsection{Motivation}

The combination of all the IoT devices, the Fog infrastructure and the container technologies, creates an heterogeneous and decentralised distributed environment. One of the major obstacles towards the complete realisation of these kinds of applications is the complexity of managing all the heterogeneous technologies across its levels:

- The first challenge is at monitoring level, specifically in providing observability to the operators on the status of the devices through monitorisation techniques. In a Fog deployment, devices are normally spread throughout a wide area, laying out a scenario with associations of devices that can be thought of as small datacenters. This changes the monitoring workflow from a centralised one, such as the Cloud, to a decentralised and federated one. In addition, monitoring such a vast and ever changing infrastructure, where the number of elements to observe is much larger, requires new ways of filtering, aggregating and storing the metrics.

- The second challenge is at the software level, particularly knowing what went wrong in our data processing workflow if it fails. This is far from trivial. Specially 
because instead of having a monolithic application running in one place, the different responsabilities are divided into loosely coupled units in the form of containers, following a service oriented architecture (SOA). The containers will be hosted in the numerous devices of the Fog deployment, complicating the root cause analysis (RCA) of the failure even further.

- The third challenge is related to the data processing up at the Cloud level. In this step, intelligent models are created through the analysis of the data gathered at the sensor level. The analytical process is powered by the different Big Data technologies that run in the Cloud, which sometimes involve the tuning of different configuration parameters to ensure their performance and correct execution. This tuning follows a different criteria depending on the type of data analitycs job, which further complicates this task to the users.

In order to solve the aforementioned challenges, users would need to possess a vast knowledge about all the technologies that are used across the different layers of the infrastructure. It is clear that there is a need for tools that facilitate the maintenance and management of such a complex stack. So far, users often follow a simple process in which they correlate certain events, such as failures or under-utilisation of the infrastructure, with certain system metrics or log entries. For instance, a low cpu usage in machines that are hosting a compute intensive task, would be an indication that the workload is not running properly. It seems logical that if we extract information from these metrics and log entries and represent them in a machine understandable way, artificial intelligence techniques can be used to extract the correlations between the data and any decision that needs to be made for the management of the system.

\subsection{Objectives and hypothesis}

In this thesis, we hypothesize that the performance and management of the distributed systems used in the Internet of Things, can be improved by using artificial intelligence models that learn from observing the behaviour of these technologies. This is based on the intuition that an AI model will be able to find correlations between events and outcomes that happen in the system in a more effective way than a human administrator. 
In order to prove this hypothesis, this thesis fulfills a series of sub-objectives summarized as follows:

1. Present the new decentralised architectures that IoT gives rise and the different technologies that form part of it, together with the monitoring tools that can be used to capture their behaviour.

2. Study the benefits and implications that an autonomic management system powered by AI can have over these decentralised systems with respect to a traditional human administrator approach.

3. Definition of the methodology used to extract relevant features from the observed metrics in the system and to build the different AI models required.

4. Design and implementation of the tools that will allow us to evaluate our previously mentioned methodology.

5. Evaluation of our tools in real-world deployments, using representative use-cases and scenarios to prove the usefulness of our approach.

\subsection{Contributions}

The main contributions of this thesis can be summarized as follows:

A definition of the different technologies that power IoT applications and their different requirements in terms of data processing. We present the context of our work to understand IoT applications in terms of data models and processing requirements. This serves as a motivation to illustrate why these technologies are needed for data processing, and their challenges in terms of system management. We also detail a series of benchmarks that will allow us to emulate Big Data workloads and to understand their behaviour. This work was carried out in collaboration with Athanasios Kiatipis, Rizkallah Touma, Pierre Matri, Michał Zasadziński, Linh Thuy Nguyen, Adrien Lebre and Alexandru Costan.

A study of the I/O calls of representative high performance computing (HPC) and Big Data analytics workloads. We complement the work of Pierre et. 
al by leveraging some of the benchmarks previously studied together with several HPC workloads to analyse the typical storage call patterns of these kinds of applications. The majority of the calls are files reads and writes, opening the possibility for improvements in the underlying storage systems through the use of object storage approaches. Even when calls that are not read or write are mapped onto the object storage, we get significant performance gains. The results allowed us to, first, understand the data access patterns of workloads often used in IoT, and second, to show how the usage of distributed storage can be simplified with a single solution for all the large-scale analytics applications. Our contribution was included as a complement to the main work carried out by Pierre Matri, Yevhen Alforov, María S. Pérez, Alexandru Costan, Gabriel Antoniu, Michael Kuhn, Philip Carns and Thomas Ludwig. It was published in (96) and (97).

FMonE: An effective monitoring solution at the Edge. We present a study of the requirements that an Edge monitoring tool should meet, based on motivating scenarios drawn from literature. Additionally, we propose the design of a monitoring framework that adapts to the particular characteristics of the Fog/Edge infrastructure. Specifically, FMonE provides support for the different heterogeneous technologies and hardware present in the system, together with the construction of geo-aware monitoring workflows that extract, filter and aggregate metrics depending on the user criteria. It also offers flexible backends for those metrics, allowing the user to choose them based on the storage and processing capacity of the device that is going to host the backend. We evaluate FMonE in a simulated Fog environment in the Grid'5000 testbed. This work was carried out in collaboration with María S. Pérez, Alberto Sanchez and Jesús Montes. It was published in (22).

\section{A graph-based root cause analysis approach for microservices architectures.}

We focus on containerization, one of the technologies that eases the deployment of software in IoT. Along these lines, we model microservice architectures as multi-attributed graphs and use graph similarity techniques to facilitate root cause analysis on them. We illustrate how graphs are a suitable model for these kinds of architectures, as they are based on containers and their interactions, modelled as nodes and edges respectively. By monitoring deployments of widely used microservice architectures, we show that the model outperforms in terms of accuracy other approaches that do not take into account 
the topology of the architecture. This work was carried out in collaboration with María S. Pérez, Marc Solé, Alberto Huelamo, David Solans and Victor Muntés-Mulero.

\section{A cross-system diagnostics method for root cause analysis based on graphs} and log analysis. We complement the work of Michal Z. et al. expanding on the previous ideas and enabling the transfer of the knowledge acquired for RCA in one microservice architecture or domain to a different one. This is done through the inexact matching of the graph representations and the inclusion of log entries as attributes of the different elements in the system. Through experimentation, it is demonstrated that it is possible to transfer the RCA knowledge acquired from a decentralized architecture, such as Cassandra, to troubleshoot a similar one, such as Kafka. This contribution was included as a complement to the main work carried out by Michał Zasadziński, Marc Solé, Victor Muntés-Mulero and David Carrera. It was published in (152).

\section{A machine learning approach to optimise parallelism in Big Data applica-}

tions. We study the important effect that tuning parallelism parameters has in the duration and energy consumption for representative Apache Spark jobs. This motivates the design of a pipeline that extracts representative features of a Spark job and then feeds them to a machine learning model, which is able to accurately predict the duration of that job. The model is used to recommend the user a suitable parallelism configuration that is able to split the tasks across the cluster in an optimal way, depending on the characteristics of the job. The whole process is evaluated through real experiments in the Grid'5000 testbed. This work was carried out in collaboration with María S. Pérez, Smrati Gupta and Victor Muntés-Mulero. It was published in (59).

\subsection{Organization of the manuscript}

The rest of the manuscript is organized in three parts:

The first part presents the context of our work, capturing the state of the art in related research areas. It consists of two chapters. In the first one (Chapter 2 ) we provide an overview of IoT. We define the concept, describe the type of applications it powers and briefly present the different layers of its heterogeneous technological stack. This allows us to identify three key technologies used in IoT: Fog computing, microservices and 
Big Data. We described them and identify the challenges related to their management. In the second chapter (Chapter 3) we study different techniques that will allow us to solve those challenges. In particular, we turn to autonomic computing as it has been used previously as a tool to manage other complex systems and can integrate the AI models we intend to use. We put a special focus on monitoring, specially challenging for Fog computing and one of the most important aspects of autonomic computing, as the monitored metrics and events will serve as inputs for our AI models.

The second part contains the contributions of this thesis. We explain the different methods that we use to monitor, manage and optimise the behaviour of the different technologies involved in the IoT ecosystem. It is divided into four chapters. Chapter 4 presents the common methodology used. It consists of a workflow inspired in autonomic computing, in which we monitor the system, train artificial intelligence algorithms with features extracted from those observations, and use the resulting models to aid the user in the decision process. We also explain how we include the user in the workflow and how our contributions reassemble a decision support system oriented to these IoT technologies. In Chapter 5 we explain the design of FMonE, the Fog monitoring tool that implements the "observation" part of this framework. We continue with Chapter 6 , where we present a system that is able to diagnose a root cause when a microservice architecture is experiencing an anomalous behaviour. The objective is to ease the management of the different containers in the Fog infrastructure. In Chapter 7 we zoom in on the upper level of the Fog structure, the Cloud. Specifically, we focus on the impact of choosing an optimal parametrisation for the Big Data jobs that analyse the data generated in the lower layers (sensors, devices, etc...).

The third part summarises the conclusions of this thesis. Chapter 8 wraps up the previously explained contributions, discusses the limitations of our work and finishes with future research lines in the field of autonomic management for Big Data, Fog computing and microservices. 


\section{Part II}

\section{Context: The IoT technological stack and its management}





\section{Chapter 2}

\section{The Internet of Things and its technological stack}

The Internet of Things (IoT) is a paradigm that has been driven by the proliferation of cheap devices able to generate valuable data, together with the new technological advancements in Big Data analytics to process it. One of the most important uses of this paradigm is the possibility of integrating and analyzing all the generated data in order to make informative decisions in fields like healthcare, traffic management, water quality, air pollution and many more (55). Smart Cities (150) is one of the most well known examples, where technology is used to improve the life of citizens. From the physical plane up to the Big Data analytics, there is a wide variety of technologies used throughout the whole cycle that elaborate a complicated technological stack. Besides this heterogeneity, IoT is also powered by a decentralised computing architecture in order to control the numerous elements involved.

In this chapter we define IoT and provide an overview of its different technologies and protocols. We introduce a decentralised computing paradigm that was created to meet the requirements of IoT applications: Fog computing. As part of this paradigm, we explain how containers have been used to deploy software in the Fog, together with the use of the Cloud to process IoT data. We finish off with a conclusion on the importance of IoT in the new digital landscape and the challenges in terms of management for all its related technologies. 


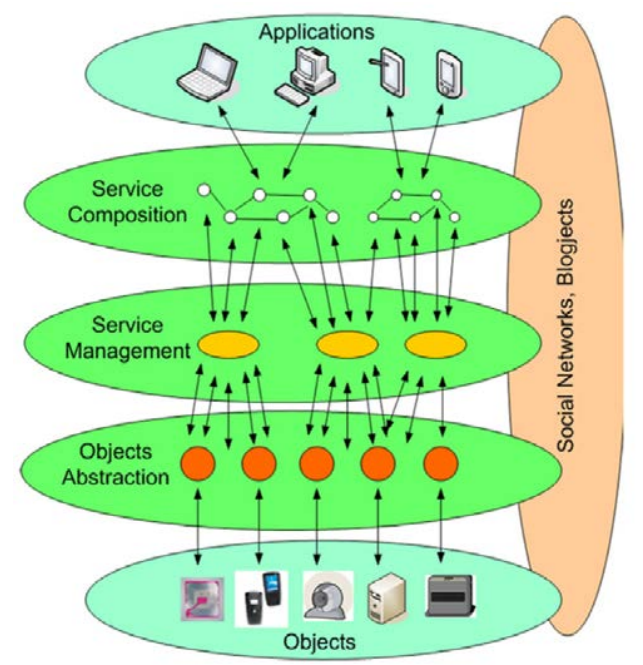

Figure 2.1: Integration of the things and the Cloud (81)

\subsection{IoT: Definition and technologies used}

The IoT is an ambiguous term that has been used in many contexts. One of the first mentions that we found is the article of Gerschenfeld et al. (48). The authors envision a network of objects that are able to connect to the Internet, creating a massive intelligent system that is able to sense and act in our environment. Additionally, IoT provides means for the things to communicate and for humans to interact with them. In order to achieve that, an architecture standardization needs to be accomplished by adapting current protocols together with the use of old and new technologies. This is far from easy and its complexity and challenges have been acknowledged already in the literature (135). The final objective is to connect the sensing things that generate the data with the powerful computing capabilities of the Cloud (20), as seen in Figure 2.1. We will briefly describe each layer in the following subsections and identify key technologies in this environment.

\subsubsection{Objects: the sensing layer}

This layer generates the data capturing the status of the world surrounding us through data. We can think about this data as measures, like the temperature or humidity registered by a sensor, or simply detecting the presence of a real world object, such as a person going through the turnstile of a train station. 
The nodes that make up this infrastructure have three main parts. The first one is a microcontroller that controls the other components of the node and does limited processing. The second one, is one or more sensors attached that transform a physical measure, such as temperature, to a digital signal. Finally, it has a transceiver to be able to send this information wirelessly.

The communication interfaces are probably one of the most heterogeneous parts of IoT (9). Sensor nodes could have a limited power supply and they often use low footprint interfaces such as Bluetooth, IEEE 802.15.4-based antennas (24) or Zigbee (44). Also longer-range interfaces, such as Wifi or a $4 \mathrm{G}$ antenna, can be installed if the power source allows for it. It is out of the scope of our work to explain each one of these technologies, as they are part of the physical layer of the communication stack. However, we believe that their heterogeneity must be taken into account in terms of connection speed, range and reliability, as they will be important factors in the infrastructure management process, which is one of the objectives of this thesis.

\subsubsection{Networking: a constrained internet protocol stack}

The sensing layer is made of devices that have limited access to power. The heaviest protocols of the Internet cannot be used and, consequently, lightweight alternatives have to be developed. The final goal is to enable the connection between the Web and the things in a seamless way. Several of these protocols are depicted in Figure 2.2. Note that some protocols are analogous to those of the Internet stack, such as COAP (19), which is also based in the REST model.

Explaining each of them is out of the scope of this thesis. However, it is worth noting that the union of all these protocols, together with the legacy ones already used in the Internet, create a vast and heterogeneous sea of protocols. An entity is needed in order to translate all the different languages spoken by the things. IoT gateways were created with this purpose in mind, acting as a bridge that would enable to communicate the different worlds inside IoT (155). They are intermediate nodes, which are more powerful than the devices in the sensing layer at the bottom, yet less powerful that those up in the Cloud. We can already start seeing here the hierarchical and technologically diverse nature of an IoT infrastructure, in which the devices at the bottom generate data, which is processed later in the intermediate and upper layers.

\footnotetext{
${ }^{5}$ https://www.slideshare.net/butler-iot/butler-project-overview-13603599
} 


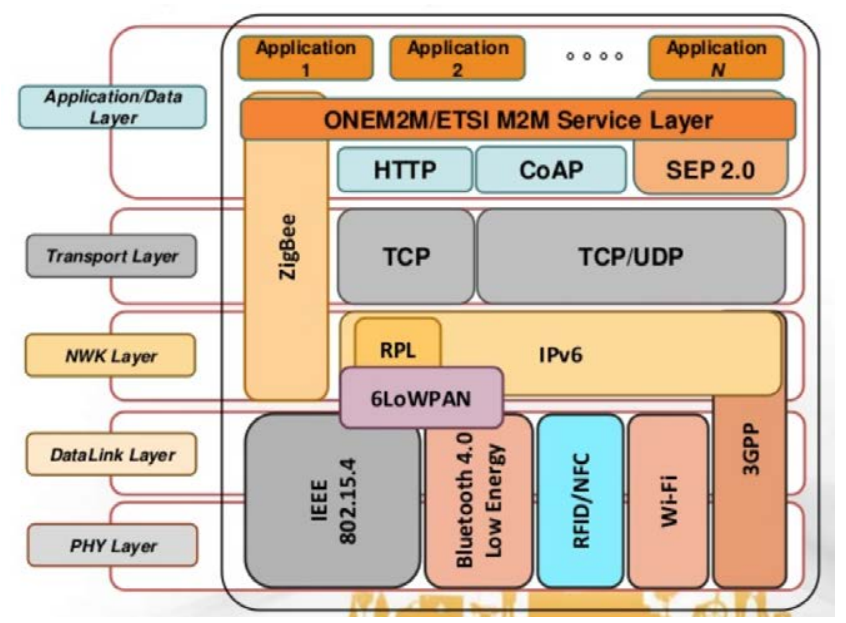

Figure 2.2: The network stack for IoT devices 5

The protocols present in the stack will allow to move the data through the different levels.

\subsubsection{Middleware: abstracting the complexity}

We have already mentioned the need for a translation between the different components of the IoT. As a general definition, middleware is a software that enables the exchange of information between two entities that would not be able to communicate otherwise. It seems logical to have this function in an heterogeneous ecosystem like the IoT. There are several reasons behind this approach. Firstly, it is difficult to have a standard for all the different devices and protocols, creating the need for a layer that ensures interoperation. Secondly, the middleware can provide a management layer from where we can keep track of the different things in the physical layer together with their data and services. Lastly, it abstracts all the complexity and heterogeneity to the user, facilitating the implementation of IoT services.

This middleware has to stand between the sensing layer and the Internet in order to act as the bridge between the two worlds. Therefore, it should be able to run in a variety of devices that could have different hardware or operating system. Containers can provide this abstraction layer, since they do not depend on any hardware architecture or operating system, enabling the easy deployment of any kind of IoT software. They cannot be used in the sensing layer, as they are made up of low computing power devices 
in which virtualization would mean an unacceptable overhead, but can be deployed in the intermediate nodes i.e. gateways (134). They also help to build portable and lightweight middleware (105).

In addition, much of the software tools that have been build for IoT follow a Service Oriented Architecture (SOA) (36). In this paradigm, a monolithic application is separated into different independent units, each one with a well-defined responsibility, which interact between them to achieve a goal. One of the parts that constitute these SOA's is a microservice, in which each unit is running inside a container. This means that instead of having a single point of computation, where all the software functions are executed, we can have many spread across different devices. More details are provided in Section 2.2.2, but we can already see the synergy between the IoT infrastructure, with numerous devices that can perform a small part of the computation, and the microservices paradigm, in which the software logic is divided across several containers. All of the above observations have led the research community to adopt containerisation as an enabling technology for the IoT middleware (15). Managing the self-healing of these containers and finding the root causes behind anomalies or performance issues in this context is one the challenges to solve as part of this thesis (Chapter 6).

\subsubsection{Applications: connecting users to things}

The final goal of the IoT is to provide applications that make use of the gathered observations to improve decision-making in several fields (31). These applications sit on the top of the whole hierarchical architecture we presented in Figure 2.1 and are the entry point of the users to the IoT as an entity. They are normally hosted in the Cloud and use Big Data technologies to analyse all the information. In fact, there are many IoT oriented Cloud services, such as Amazon Web Services IoT or Azure IoT Hub, which act as platforms for these applications (56). The platforms also offer functionality like discovering the different resources and things, configure them and even push decisions, as previously mentioned. Researchers have also proposed architectures in this line of work (85), apart from the more industry-oriented solutions.

However, this raises the question of whether we need to get back to the Cloud to make some decisions. This is not the case in some situations, like a traffic light deciding if cars driving in one direction should continue or stop depending on the surrounding traffic (130). In this case the decision is made with data and observations of a particular 
region that spans the proximity of the traffic light and there is no need for it to travel to the Cloud, which will have higher round time trips. This observation drives the design of a new decentralised computer paradigm called Fog computing, which we describe in Section 2.2.1. In Section 2.2.3 we elaborate further on Big Data technologies, as they will also be one of the targets of the thesis (Chapter 7).

\subsection{Enabling technologies for the Internet of Things}

The previous section presented the general context of IoT and the different parts that constitute it. From all the layers there are three key technologies that are going to be the center of our thesis: Fog computing, microservice architectures and Big

Data technologies. We explain each of them in the following subsections:

\subsubsection{Fog Computing}

So far we have seen how IoT involves heterogeneous technologies, protocols and gateways able to tie together the Internet and the things. As for the applications, we described how they make use of the information that sensors collect and that they can be hosted in the Cloud, as a way to process all the data deluge. We also mentioned that sometimes, there is no need for all this information to travel from where the sensors reside, also known as the Edge (123), to the Cloud. This will avoid long round time trips of the information, specially if the Cloud service is hosted in a distant location. It will also prevent any bottlenecks in the network, which can be usual in scenarios with thousands of sensors generating data (154).

All of the previously mentioned characteristics gave raise to a new type of distributed infrastructure known as Fog computing (138), so called because it aims to bring the clouds closer to the ground level and near the sensors. Fog computing consolidates all the previous ideas of processing, filtering and aggregating data closer to where it is generated, thanks to intermediate nodes that sit between the Edge and the Cloud. These nodes are also known as Fog nodes (91) and they are the ones acting as IoT gateways and providing all the middleware functions needed. Figure 2.3 illustrates a simplified Fog architecture. 


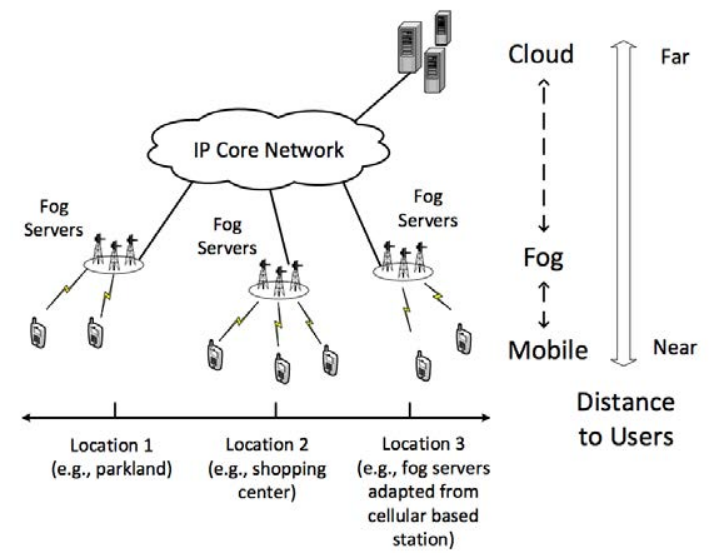

Figure 2.3: A simplification of the Fog architecture (90)

\subsubsection{Characteristics of the Fog}

There are many publications that provide a good review on the characteristics of the Fog (137; 148) and its decentralised nature. Here we present a small summary in order to understand the management challenges that come with these type of infrastructure.

- Low latency: processing any requests of clients and/or data should be done fast, as many of the Fog applications have low-latency requirements i.e. augmented reality, smart retail (115), etc. Keeping the Fog nodes close to their clients and the data they need to use, helps to satisfy these requirements

- Geo-distributed nodes: In the same line as we have seen so far in IoT, the Fog nodes are spread throughout a wide geographical area. This is a stark contrast with the Cloud, where everything is centralised in a single location and nodes have access to high speed connections between them.

- Mobile nodes: One of the additional design principles of the Fog is to leverage devices that are mobile, such as phones or nodes installed in trains or drones (64), to collect the data of sensors in their proximity, as many of them do not have long range communication capabilities.

- Heterogeneous nodes: Nodes in the Fog can be from different manufacturers and of different types. This will imply a large variety of operating systems and hardware present in the nodes. 
- Large number of nodes: Since Fog nodes are much cheaper that those on the Cloud and the paradigm is based on having as many servers as possible near every client and data source, the infrastructure will end up having a large number of servers, instead of a few and powerful ones, as in the traditional Cloud.

- Volatile nodes: There are many reasons why a node might stop functioning properly. It could lose connectivity due to the previous mobility reasons and the unreliable wireless technologies they use. It could also run out of power or just be switched off in the case of mobile phones.

- Limited computing and storage power: While the Cloud can be considered as an endless pool of storage and computing power, Fog devices are much more constrained in terms of hardware.

- Hierarchical organisation: We have already mentioned the hierarchical tree-like structure of the Fog, with the Edge at the bottom collecting the data and the Cloud in the upper part performing the big data processing.

From the previous characteristics, we can already see that they are unstable and very changeable infrastructures, compared to more stable ones, such as the Cloud. That means we need new monitoring models that provide observability and control over these kinds of infrastructures. We undertake this task as part of our thesis in Chapter 5 .

\subsubsection{Microservice architectures}

One of the enabling technologies for IoT and Fog computing is containerisation. It allows to package an application without worrying about any dependencies, or the platform in which it is going to run in. This is an important requirement in an environment such as IoT, where we have different devices from different vendors. Also it eliminates the burden of installing all the dependencies in the thousands of nodes that might comprise the Fog.

Containerisation, in simple terms, is a feature of the operating system kernel that isolates the machine resources used by a given program. In this way we can divide the hardware available between several programs and isolate them from the rest. This 


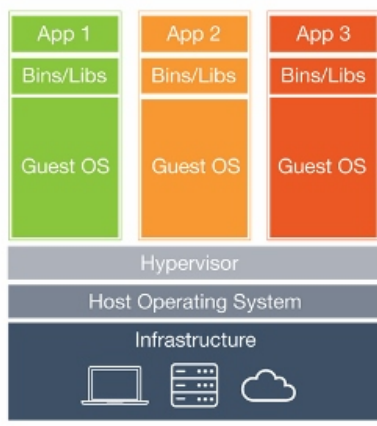

Virtual Machines

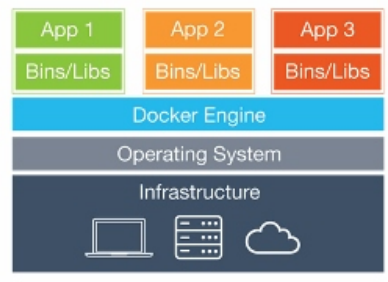

Containers

Figure 2.4: Comparing containers to virtual machines ${ }^{7}$

concept is very similar to virtual machines (VM's), another type of virtualisation technique. VM's, however, create virtual hardware through an hypervisor, while containers use the OS kernel of the machine they are running in. Although sharing the kernel could turn into a disadvantage in terms of security, containers have the following advantages:

Lightweight: While a VM includes the operating system and additional features that we might not use, containers use the OS of the machine they run in. This eliminates all the overhead issues of VM's: occupying space in both memory and disk and having a higher impact in CPU utilisation (76).

Elasticity: A container can be started in a few seconds, as there is no virtual operating system to boot. This allows a infrastructure to react quickly to changes in the workload (8).

Better resource utilisation: We mentioned before that a portion of the machine resources is assigned to a container. However, if that container is not using its resources, they can be allocated to any other container, which translates in better resource utilisation 6

A comparison between the VM stack and the container stack is shown in Figure 2.4. There are platforms that leverage certain features of the kernel to create and boot all of these containers in an easy way. Docker is one of these platforms and without any doubt the most popular amongst user 8 .

\footnotetext{
${ }^{6}$ https://docs.docker.com/config/containers/resource onstraints/ /

${ }^{7}$ https://www.slideshare.net/Docker/docker-birthday-3-intro-to-docker-slides

${ }^{8}$ https://www.datadoghq.com/docker-adoption/
} 
In our case, the most interesting part of Docker is that it enables the Fog computing paradigm (71) because of two reasons. Firstly, Fog nodes do not need any previously installed dependencies. Since they are packaged inside the container, we can run the software on any of the many nodes that comprise the Fog, without any constraints on the hardware architecture or dependencies installed, as long as a Docker daemon is running on the node. Secondly, containers can be booted up quickly. As soon as a node joins the Fog network, a container with the relevant software can be up and running. This is important when we have mobile nodes, or devices that can be switched off and on. Many of the Fog prototypes and small scale deployments done in research involve Docker as an enabling technology (102; 106).

However we need to take into account that the Fog is a distributed infrastructure with several hosts. In many cases, an application is not made of a single container, but of many containers that collaborate with each other to fulfill a common task. Container ochestrators like Kubernetes 9 or DCOs 10 take care of this task. This is normally done by writing a YAML or a JSON file that will be sent to the orchestrator. The file contains information about the containers needed, their parameters, number of replicas, constraints (e.g. type of host they should run in, number of containers on each host, etc.), and any additional elements that represent the desired status of the containers in the cluster. Orchestrators have also been used in Fog proof-of-concepts (117). This way of architecting software, where a system is deployed as a set of small services that interact between each other through lightweight mechanisms, such as REST requests or RPC based API's, is known as a microservice architecture. The Fog is a suitable infrastructure to host this kind of architecture, as the different microservices can be allocated throughout the numerous devices, effectively dividing the computing burden of the application.

This paradigm has already been used to implement IoT utilities (141) and in the context of this work, we believe it is one of the key technologies to take into account. The management of containers and its interaction forms an integral part of this thesis. Although breaking the application into simpler parts simplifies the development process and provides a more robust system, it also complicates the troubleshooting and

\footnotetext{
${ }^{9}$ https://kubernetes.io/

${ }^{10}$ https://docs.mesosphere.com/1.11/overview/what-is-dcos/
} 
identification of the root cause of a problem in the system. We deal with this topic in Chapter 6 .

\subsubsection{Big Data Analytics}

The final purpose of the Internet of Things is to extract valuable insights and models out of the information coming from the different things and sensors. Big Data is a term that refers to a type of data with a set of special characteristics that cannot be processed by traditional data analytics software. The data generated by the IoT possesses this collection of characteristics, which are originally known as the three V'11. volume, variety and velocity. Volume, because of the tremendous amount of data sources (sensors, cameras, GPS, etc.). Variety, because the data can have different formats. It can be unstructured data such as images or video, or just periodic measures of the temperature or humidity of the environment. Velocity, because this data can be generated at high speed and it has to be processed in almost real time to enable quick decisions. Big Data analytic platforms were created to process this type of data and they are an integral part inside any IoT application. We briefly analyse the common characteristics of these platforms in the following sections.

\subsubsection{Processing and storage requirements for IoT}

In order to understand the criteria behind the design choices of these technologies that we seek to optimise and control, we need to examine the different processing and storage requirements of IoT.

Low-latency processing and storage: IoT applications and low latency are heavily linked. The potential value that can be extracted from raw data is immense, but for it to express its full potential, one must be able to analyze and derive insight from it in almost real-time. In many cases, the longer the time-to-decision, the more the information loses value.

Batch Processing: IoT sensors and devices generate huge amounts of data that are preferably stored in its entirely. In order to process this kind of data that has been stored for a period of time, batch processing jobs are used. This processing

\footnotetext{
${ }^{11}$ Sometimes this is referred too as the 4 V's with veracity as an extra characteristic
} 
is normally done in blocks or batches of data. Batch jobs usually take a long time to complete, depending on the size of the data and the complexity of the computation. Because of the high demand of computational power, these kinds of jobs are executed in the Cloud as the supporting infrastructure. Remember that the Cloud is located at the highest level of the Fog hierarchical set-up, therefore we can say that most of the batch jobs are located there. An example would be analyzing all the measures of a temperature sensor for one week or training predictive models with a given dataset.

Stream Processing: We have already mentioned that in many applications the value of the data resides in its freshness. Deriving insights or making complex decisions in real-time out of a big stream of data, is one of the motivations behind stream processing or also called complex event processing. In contrast to batch processing, the data is analysed as it arrives in an event-like manner. An example could be a self-driving car, in which data coming from different sensors is analysed as it arrives and combined in order to make the proper driving decisions. Note that this analysis does not have to reside in the Cloud, but it could be hosted near the things/Edge in order to increase the freshness of the data that arrives.

Distributed Storage: The amount of data generated by IoT applications far exceeds the storage capacities of a single machine, hence, the storage of the data has to be done across several machines on a distributed infrastructure. Storing such vast amounts of data is challenging. Yet, storing the data is a requirement for many applications. It should be able to handle writes at the peak data generation velocity, in order not to lose precious incoming data. Also, it should provide the required read performance for the data analytics systems in order not to cause data starvation and waste valuable computing resources. Depending on the precise requirements of the application, multiple storage paradigms can be considered. For instance, object stores are ideal for storing the raw data originating from the sensors, while columnar stores are best suited for structured data. Although some solutions have been proposed to unify all of these different models (96; 97), the key takeaway is that different types of applications require different storage models. 
Geo-distributed data analysis: As the data gathered from many sensors could possibly be stored in datacenters around the globe, some limitations could arise on the communication between these datacenters. Data analytics that exploit all the data at the same time, in order to derive meaningful results, could hinder bandwidth limits, regulatory constraints, problematic runtime environments or monetary costs (153). Computation procedures could be distributed, in order to take advantage of more than one datacenter (68) and improve the job completion time. This all requires a geo-awareness of the different devices doing the computation, as we will have a series of circumstances of conditions that will not otherwise happen in a centralised environment, such as HPC or Cloud.

\subsubsection{Data analytics platforms}

Big Data Analytics (BDA) has the potential of answering to all of the computational challenges of IoT applications. In the last few years, many BDA platforms (127) have been created. Some of the most popular are MapReduce (37), Apache Spark (149), Apache Flink (26) or Apache Kafks 12 . Although each one provides different features, they all share the same design principles:

- Horizontal Scaling: In order to increase performance, the workload is distributed across multiple commodity servers. Following the divide and conquer principle, the huge datasets that need to be processed can be split into smaller chunks and processed in parallel in each server.

- Fault tolerance: If one of the components fails, the system should be able to keep running properly and finish the data processing job.

- High I/O capabilities: The amount of data to be ingested is tremendous in relation to other distributed systems, such as the Grid or HPC. BDA platforms should optimise this aspect as much as possible, normally by moving the computation to where the data is or by keeping data in memory.

They have been used in many IoT and Fog computing proof of concepts. Spark was used by Alsheikh et al. (11) to propose a deep learning framework inside a context where computation is made by mobile devices. The article reveals some of the

\footnotetext{
${ }^{12} \mathrm{http}: / /$ kafka.apache.org/
} 
challenges for this kind of distributed computing, such as the large scale, the volatility and the heterogeneity of the devices. OpenTOSCA (43) is a project inspired by the Industry 4.0 data-driven approach, in which Flink is used to provision an environment able to ingest all the information generated. It has also been used in ambitious project just as WAZIUP, a project born from the collaboration between Africa and the European Union, to bring the advantages of IoT to the rural activity in Africa (29). The data processing model of Kafka fits clearly inside the IoT scenario and is one of the key technologies used for stream processing with many producers and consumers of data (74).

From all these use cases and proof of concepts we can observe how BDA's are a key element in Fog computing, as they provide the computational power to extract insights from the data. Yet, their complexity can hinder their deployment, usage and performance. In Chapter 7, we explain how we ease this task to the users, and we will get into more details regarding their architecture and components.

\subsubsection{Benchmarking Big Data analytics}

So far we have given an introduction to some of the Big Data technologies that form part of many IoT application deployments and that we will use in the elaboration of this thesis. Remember that our objective is to optimise and ease the management of these distributed infrastructures. In order to achieve that, we need to understand their resource usage and behaviour. Therefore, an important requirement is to simulate representative workloads in our experiments. Here we introduce some of the benchmarks we will use to simulate the jobs and processes running in these kinds of infrastructures. Their usage has two purposes. Firstly, they will work as a baseline to measure any performance improvements we could get through our decision support system. Secondly, they can be used to gather real resource usage patterns and metrics for BDA workloads. We will briefly describe the most relevant ones in the context of this work, with a special focus on that ones that emulate IoT workloads.

Linear Road (12): Linear Road simulates a toll system for the motor vehicle expressways of a large metropolitan area. The benchmark specifies a variable tolling system for a fictional urban area, including accident detection and alerts, traffic congestion measurements, toll calculations and historical queries. Linear Road is 
designed to measure how well a system can meet real-time query response requirements in processing high volume streaming and historical data. This benchmark is suited to emulate smart transport use-cases.

RIoTBench (125): The Real-time IoT Benchmark suite has been developed to evaluate Data Streaming Processing Systems (DSPS) for streaming IoT applications. The benchmark includes 27 common IoT tasks, classified across various functional categories and implemented as reusable micro-benchmarks. It further proposes four IoT application benchmarks composed from these tasks, that leverage various dataflow semantics of DSPS. The applications are based on common IoT patterns for data pre-processing, statistical summarization and predictive analytics. These ones are coupled with four stream workloads sourced from real IoT observations on Smart Cities and fitness, with peak stream rates ranging from 500 to 10,000 messages/second and diverse frequency distributions. DSPSs are key elements in the IoT context. RIoTBench can be used to evaluate them, especially in analyzing and making real-time decisions based on data coming from multiple sources.

SparkBench (87): SparkBench is a benchmarking suite specifically for Apache Spark. It is composed of a representative and comprehensive set of workloads, belonging to four different application types that are currently supported by Apache Spark, including machine learning, graph processing and SQL queries. The chosen workloads exhibit different characteristics with several system bottlenecks: CPU, memory, shuffle and IO. Hence, SparkBench covers three categories of algorithms widely used in IoT applications and that can be used to evaluate these algorithms.

BigBench (49): BigBench is an end-to-end Big Data benchmark proposal based on a fictitious retailer who sells products to customers via physical and online stores. The proposal covers a data model, a data generator and workload descriptions. It addresses the variety, velocity and volume aspects of Big Data systems, containing structured, semi-structured and unstructured data. Additionally, the data generator provides scalable volumes of raw data based on a scale factor. This allows BigBench to be used to test the batch processing aspects of IoT applications. 
IoTABench (13): The IoT Analytics Benchmark (IoTABench) aims to bridge the division between Big Data research and practice, by providing practitioners with sensor data at production scale. The initial implementation of IoTABench focuses on a single use case, specifically the smart metering of electrical meters, but the benchmark can be easily extended to multiple IoT use cases. The benchmark can generate a large dataset (22.8 trillion distinct readings, or $727 \mathrm{~TB}$ of raw data) with realistic properties using a Markov chain-based synthetic data generator. Hence, this benchmark is ideal for evaluating real-life IoT applications, which are based on sensor data.

YCSB (32): The Yahoo! Cloud Serving Benchmark (YCSB) is an open-source and extensible benchmark that facilitates performance comparisons of Cloud data serving systems. It models systems, based on online read and write access to data, while it provides a set of workloads that generate client requests to different backends like Cassandra (83), HBase (47) or MongoDB ${ }^{13}$, YCSB is easily customizable and allows end-users to define new types of workloads, to configure the clients with different parameters as well as to add new back-ends. YCSB can be used to evaluate Cloud services, which are an essential part of IoT applications, ranging from open data access APIs to use cases related to health, social networking and public services.

BigDataBench (142): BigDataBench is an open-source, multi-discipline benchmark, coming from common research and engineering efforts from both industry and academia. The current version of the benchmark models five typical and important Big Data application domains: search engines, social networks, e-commerce, multimedia analytics and bioinformatics. In total, it includes 14 real-world data sets and 34 Big Data workloads. Moreover, it provides an interesting set of workloads in both stream and batch implementations.

Intel HiBench (66): HiBench is a realistic and comprehensive benchmark suite, which consists of a set of programs including both synthetic micro-benchmarks and real-world applications. The benchmark contains a set of workloads for Hadoop and Spark, as well as streaming workloads for Spark Streaming (149),

\footnotetext{
${ }^{13}$ https://www.mongodb.com/
} 
Flink (26), Storm and Gearpump. It helps evaluate different Big Data frameworks in terms of speed, throughput and system resource utilization on a set of workloads relevant to several IoT applications.

\subsection{Conclusions: Three key technologies for IoT}

Although different definitions are considered for the term of IoT in the literature, they all envision "a world where physical objects are seamlessly integrated into the information network, and where the physical objects can become active participants in the business process" (57). In this chapter we have provided a global view of the technological ecosystem that supports the IoT vision. This will serve as a context for many of the challenges in terms of infrastructure management that we are going to undertake during this thesis. We pinpoint several key observations drawn from this chapter.

There is a need for middleware that provides observability into the Fog infrastructure: As many of the system management decisions will be based on the status of the elements involved, we need monitoring tools that enable the collection and storage of metrics at the different levels of the Fog. The monitoring tools should adapt to the particular characteristics of these decentralised architectures. This will be our first contribution in Chapter 5.

Containerisation is an enabling technology for the Fog: The heterogeneity of the hardware and the need to deploy software in a simple manner, make containers one of the key technologies to achieve this task. Not only that, but a microservices-architecture would allow to decompose the different responsibilities of one application across many devices that perform a well-defined task. This changes completely not only the development process, but also the management and maintenance of the application. Facilitating these two tasks to the user will be our second contribution in Chapter 6 .

The data collected at the physical layer is used together with Big Data platforms to build accurate decision-making models: As we are capturing the behaviour of the real world though measurements that come from sensors and the identification of the different things connected to the Internet, we will be able 
to build a global intelligence network where many elements are connected. This however requires the processing of huge amounts of data coming from different sources. Big Data technologies are used at the Cloud level with all the incoming information to create those models (70). The processes at this level also need to be optimised and controlled by the user. This task will be our last contribution in Chapter 7.

A visual summary of both the challenges and context described until now is depicted in Figure 2.5. In the next chapter we will delve into the techniques that we will use to solve those challenges.

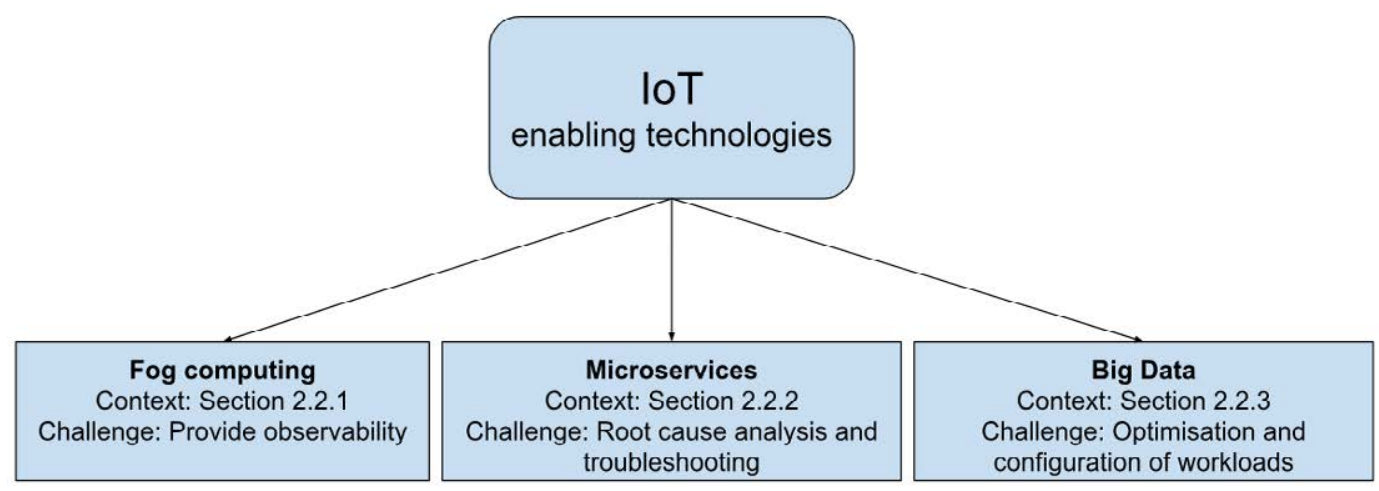

Figure 2.5: Context and challenges related to IoT enabling technologies 


\section{Chapter 3}

\section{Autonomic Management of the IoT technologies}

New decentralized systems like the Fog, could make a connected world a reality by linking the things that surround us to the Internet. However, the enabling-technologies create a complex environment which is hard to control at times, and can lead to a lack of awareness by the user of what is happening in the infrastructure. Even when all the indicators show an expected behaviour, it does not mean that the system is working at its near full performance. Technologies such as containers and Big Data technologies, expand even further the decision space and the number of elements to take into account. Human intervention in this field needs to be enhanced through the use of autonomic computing techniques to improve decision making.

Throughout this chapter we will describe the pillars of the autonomic computing concept and we will describe previous work on automatic optimisation of systems through statistical techniques. These concepts will be applied on the three key technologies previously introduced. In particular, self-healing for microservices, selfoptimisation for Big Data platforms, and monitoring adapted to a Fog infrastructure.

\subsection{Autonomic Computing}

As systems and software become more and more complex, autonomic computing advocates for systems that are able to manage themselves depending on the current 
conditions (65). The Internet and the interconnection of the numerous devices in our environment are mentioned as an additional factor contributing to the difficulty of this task. This is precisely the current setting of the Internet of Things. Kephart et al. (77) built on these ideas to reason why configuring, optimising and maintaining complex architectures will become impossible even for the most skilled system administrators. They divide the characteristics of an autonomic system into four different categories:

- Self-healing: Roles like the site reliability engineers take care of troubleshooting any failure in the infrastructure or the software running on them. Certainly, it is unfeasible to rely only on human intervention to solve the many problems that can arise in an infrastructure made of million of devices interconnected. Not only an autonomic system can make faster decisions, but it can have seamless access to a great amount of stored information that would be impossible to process by a human expert.

- Self-optimisation: Complex software comes with different configuration options, providing the user some degree of flexibility in their usage. However, the user might not always be aware of this option, or even if they do, they might not be able to choose the right configuration for their use case. This can have a great impact on the performance of the application and it can be handled in an intelligent way by the autonomic system.

- Self-configuration: An autonomic computing system is able to install, configure and prepare for production the different components. Not only that, it should be able to add any additional pieces needed in the system and integrate it with the existing ones.

- Self-protection: A system should be able to automatically detect any intrusion or attack against the system, such as malicious activity or cascading failures.

A survey on the topic (67) explored the models that were proposed to fulfill this difficult task. The main approach is named MAPE-K, standing for monitor, analyse, plan, execute and knowledge. In this model, we have a managed element, which can be anything from a small device, to a database or a whole cluster of machines, coupled with sensors that monitor this managed element. The decisions are made by an autonomic 


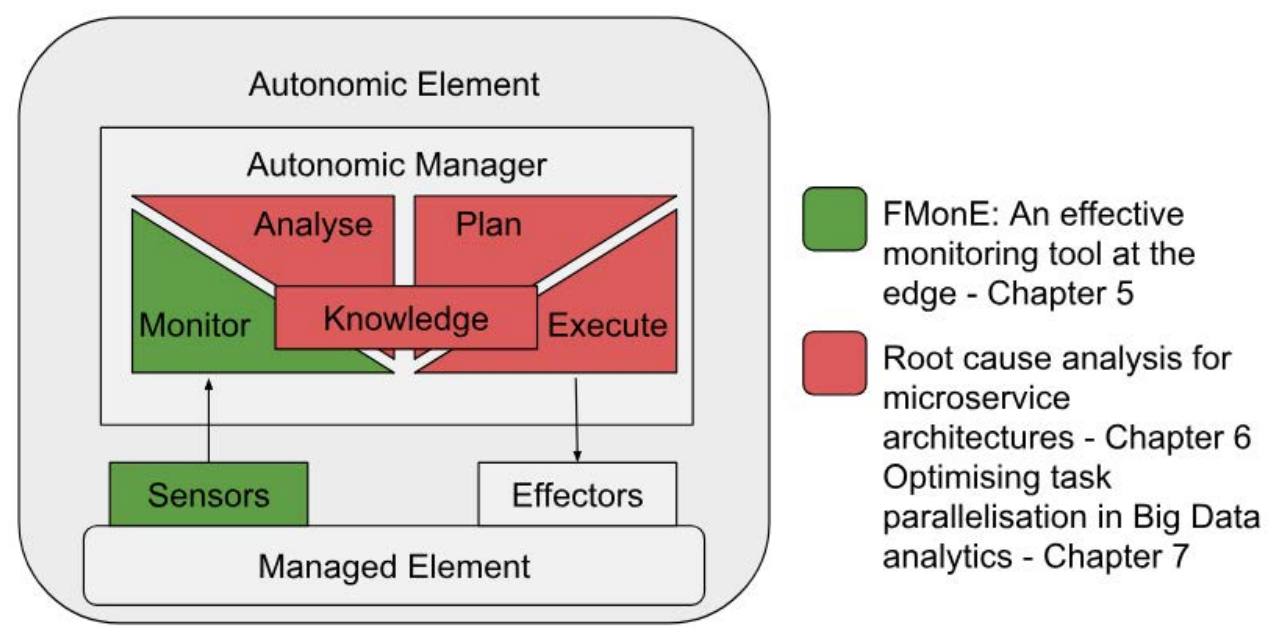

Figure 3.1: The MAPE-K model for autonomic control together with our contributions (67)

manager that analyses the information provided by the monitors to build plans that are executed by the effectors. The whole process creates a knowledge base that is going to guide the automatic manager. During this thesis we will take this approach as a reference, and implement parts of it to manage the technologies presented in the previous chapter. The whole MAPE-K model can be seen in Figure 3.1 together with the specific areas that we use and implement in our contributions. In the following subsections we are going to explain more in detail the aspects of autonomic computing that are going to be used in this thesis, as well as related work in the area.

\subsubsection{Self-healing}

Self-healing is defined in Debanjan et al. (51) "as the property that enables a system to perceive that it is not operating correctly and, without (or with) human intervention, makes the necessary adjustments to restore itself to normalcy". Self-healing requires the definition of several terms and challenges related to it. One of them is the necessity of defining what is considered a healthy or unhealthy state, in order to initiate the healing process when necessary. It can be defined based on some fixed-thresholds but normally they vary as the system evolves. This state can be checked constantly or periodically, by time intervals. Several strategies can be used to detect the malfunctioning of a system: 
- Missing Component: The strategy is based on finding out if one of the pieces of the system is missing. An example of this kind of approach would be the periodic health checks that ensure that an element is up and running.

- System Monitoring: This model advocates for probing the different components, extracting metrics and using them to detect failures. In this way, we can have more detailed information of the system and not based our criteria only in the binary concept of an element that is running or not. Problems like a performance drop or an overloaded system can be detected through metrics with this approach. This is the strategy we are going to follow in our approach, as the heterogeneous technological stack of the Fog and microservices requires the collection of several metrics from many elements to detect any anomalies.

- Foreign element: This is the opposite of the first point in the list. In this case we detect any unexpected element and act accordingly. It is heavily linked to malicious attacks and self-protection.

After the problem has been detected, there are several ways to heal and recover the system:

- Redundancy techniques: By replicating failed or dead components, the effect of the failure can be diminished. This technique is commonly used in container orchestrators, relaunching any container that fails or does not pass a health check.

- Architecture models: They are more sophisticated than other approaches, allowing for global decisions to be made by considering all the elements involved, which is the case of distributed systems. That means that the reparation will be based on establishing the root cause of the problem and executing a plan based on it. Our self-healing approach to microservices follows this model, as we will see later in our contributions.

There is a corpora of work that deals with self-healing distributed systems (see (10, 33; 98) ). However, there is little work on the microservice side, which is the technology of interest from the point of view of this thesis. Toffeti et al. (133) propose an architecture to self-manage microservices which also has self-healing capabilities. It does so by having a complete decentralised approach to manage the different containers in 
the system in contrast to centralised ones, such as Kubernetes. In App-bisect (73), the authors propose a system that is able to solve an error in a new deployed version of the architecture by detecting the new parts and redirecting the traffic to previous versions of the conflicting microservice. None of this approaches tries to find the root cause of the problem and they use redundancy techniques instead. One of the contributions of this thesis is the development of an architectural model able to find a root cause, which can be used to build contingency plans and to prevent the occurence of the same problem in the future. The only work we found in this direction for microservices is Sieve (132), which provides a platform that gathers metrics from the elements of the microservice, filters them and finds dependencies between components in the system. However, root cause analysis is only used as a use case to show the benefits of Sieve. As one of the key steps in the self-healing approach, we will talk briefly about root cause analysis in the following subsubsection.

\subsubsection{Root Cause Analysis}

Root Cause Analysis (RCA) is one of the key elements in the process of self-healing. As the name implies, RCA is a process in which the origin of a problem or event is identified in order to apply the right contingency plan or to find ways to prevent them in the future.

There is a large body of work related to Root Cause Analysis (RCA) (see (128; 144) for extensive surveys). All RCA techniques can be classified according to different characteristics. One relevant classification to frame our technique is on whether the diagnostic model knows the causality structure of the diagnosed system (sometimes referred as model-based approaches), e.g., (75; 120). Obviously, these techniques provide results according to the quality of the models they use and, by definition, they require large amounts of domain knowledge to be built and maintained.

On the other side of the spectrum, classification-based approaches, e.g., (38, 129) (sometimes called model-free), typically generate some internal model using Machine Learning (ML) techniques, trading the need of explicit domain knowledge by having lots of a lighter-weight type of knowledge (i.e., classification labels).

Graphs are an abstraction that is used by RCA in many ways, mainly by using Probabilistic Graphical Models (PGMs) (80) as (causal) models for diagnosis (78; 122) or as underlying descriptive model of the system to diagnose. Other uses of graphs 
in RCA have been rather limited to anomaly detection (6) and building alternative (non-standard PGMs) graph models (94). In our case, and as we will see later in our contributions, we will use graphs as an abstraction to model microservices and perform root cause analysis on them.

\subsubsection{Self-configuration}

Fog computing is a decentralised system in which numerous heterogeneous technologies and protocols are used. The configuration of the elements involved in such an environment is one of the most important challenges. The success of IoT depends on its adoption as a standard technology and its integration on our everyday lives. In this case, self-configuration would prove to be a key characteristic in the process of plugging in an IoT device and using it straight away. As described by Chatzigiannakis et al. (30), the device should be able to connect to the Internet on its own, learn about its context, and announce the different services it provides. We will not expand on the field of self-configuration, as the contributions of this thesis do not cover this topic. Still, we present it here for completeness purposes and to have a full picture of the autonomic computing concept.

\subsubsection{Self-optimisation}

In many cases, configuration settings can have a huge impact on the performance of an application. Distributed systems have made great advancements, but they are still very complex, requiring the use of several configuration parameters, in order to gain the maximum performance. Optimising the system by automatically finding the correct combination of these parameters is called self-optimisation. It has been widely explored in the literature. Some of the most complex systems to configure in an optimal way are Big Data Analytic platforms, partially, due to its use of the Java Virtual Machine (JVM), which has several adjustable parameters itself. In fact, there are studies (119) that detail how changing the heap size or the garbage collection method of the JVM can affect performance. On top of that, Big Data platforms usually have their own tunable parameters. In the following subsections we describe some of them for Hadoop and Spark, two of the most popular Big Data platforms in which researchers have put most of their efforts, and two technologies commonly used for IoT related applications. 


\subsubsection{Optimisation of Hadoop}

Hadoop is one of the most used Big Data frameworks in the Cloud and a technology that has received much attention, specially the tuning aspect of it. AROMA (84) is a system that is able to learn through machine learning techniques, which resources to allocate and the best configuration for a given MapReduce Job, in order to ensure that deadlines are met and save in infrastructure costs. In the same vein, the ALOJA project uses machine learning to provide a platform that is able to predict the impact of different configuration parameters and hardware choices in the execution time of a job. The users can explore all the data available from different executions through the ALOJA web-site (16). Other approaches that use machine learning to optimise Hadoop make use of a noisy gradient algorithm (82) to predict the execution time and tune accordingly. A different line of thought is to use what-if rules in order to make the configuration decisions. The work of Herodotou et al. (60) includes, firstly, an explanation of the what-if engine and the profiler that collects the statistical information used as an input. This serves as the basis for Starfish (61), an auto-tuning system for MapReduce that not only optimizes the parametrisation of the workload, but also provisioning, scheduling and data layout.

\subsubsection{Optimisation of Spark}

The irruption of Spark as a better alternative to MapReduce jobs, started a research line in which self-optimisation techniques were applied to the particular characteristics of this widely used data analytics platform. From the JVM aspect, Mem-Tune (147) is able to dynamically change the size of both the JVM and the Resilient Distributed Dataset cache, which is the abstraction that Spark uses to persists data in memory while analysing it. It also prefetches data that is going to be used in future stages and evicts data blocks that are not going to be used. In (108), the authors perform several runs of a benchmark and iterate through the different parameters of Spark to determine which ones are more important. With the experience acquired from these executions, they build a block diagram through a trial and error approach of different parameters. The obvious drawback is that the application has to be executed several times and this process repeated every time the input size changes. In latter chapters, 
we will present our contribution in this particular field as we also seek to optimise the Big Data side of IoT applications.

\subsubsection{Self-protection}

Self-protection is normally associated with intrusion detection systems (IDS). Securing distributed systems, such as the Fog, is of vital importance, specially when they provide services to clients outside their network. One of the strongest research areas in IoT is intrusion detection with several surveys on the topic (151). Security is specially important in devices that are part of the physical infrastructure surrounding us. Yet, the types of attacks and networks that support the IoT are completely different from traditional settings. Pongle et al. (111) propose a method designed for energy efficiency, in which light computation is performed on sensor nodes and the heaviest load is offloaded to an IPv6 router, following the typical hierarchical structure of the Fog. Another approach that deals with the new security landscape in IoT and Ipv6 is the work of Shreeniva et al. (124). They take SVELTE (116), an already existing system that detectes routing attacks, and extend it to use a metric that represents the quality of the communication between neighbours. In addition, it considers the geographical position of the nodes to have a better context of the attack. Finally, we have to highlight that some solutions make use of machine learning techniques to build the model that detects malicious attacks. Meidan et al. (99) use autoencoders to detect botnet attacks and evaluate the system in a real IoT environment. Same as with self-configuration, self-protection is not one of the main focuses of our thesis, and we present it here to have the complete picture of autonomic computing in general and self-protection for IoT in particular.

\subsubsection{Degrees of autonomy}

We mentioned that the autonomic computing model is going to build plans related to the administration of the system in different aspects. Those plans can be applied following different degrees of autonomicity. These levels range from 1 to 5:

- Level 1: This is the most manual level. Here the management process is performed completely by the operators and the only tools available are those monitoring systems that provide information such as metrics or logs. 
- Level 2: A more advanced step in which the operator is still making all the decisions but more sophisticated visualisation and analysis techniques are provided as insights for the user. This is a level commonly implemented by industry monitoring tools, such as Sysdig 14 or Netsi 15 , which show a microservice architecture as a graph with all the containers and their interactions.

- Level 3: In the third level, the system has enough intelligence to propose actions that the user may implement or not. It accelerates the whole process as the decision making is powered by correlations found automatically by the system. Some industry tools also implement this level as an additional feature.

- Level 4: It leverages the tools available in the previous level to act automatically based on certain rules that the user can provide. There is still human interaction, but it tries to minimise it as much as possible.

- Level 5: In the final level, everything is automatically managed, based on highlevel directives, such as business rules or general policies.

Organisations might choose the level that adapts better to their needs, depending on the criticity of the system and its scale. As we will see later in Chapter 4 , where we present our general methodology, our contributions can be placed in the third level of autonomicity, as we want to include the user in the process.

\subsection{Monitoring as a key element of autonomic computing}

As previously seen, monitoring is the first step inside the MAPE-K workflow. From traditional clusters to modern Cloud and Fog systems, monitoring has always been an important aspect of distributed system analysis and management. Different approaches and tools have been applied at different levels of the distributed environment, often combining their efforts. In the context of this work we have analysed three big groups of monitoring tools. Firstly, monitoring tools for Fog computing, which is the technology in which our research focuses on. Secondly, monitoring for Grid computing, since it is also a decentralised system that uses low computing-power devices to complete a

\footnotetext{
${ }^{14}$ https://www.sysdig.com/

${ }^{15}$ https://netsil.com/
} 
specific task and, therefore, technically relevant to the discussion. We finish up with Cloud monitoring, the top level of the Fog hierarchical structure, since Cloud precedes Fog and has more specific technologies available.

\subsubsection{Fog monitoring}

Due to the recent appearance of Fog computing, there are not many monitoring tools that adapt to the particularities of it.

In Mohamed et al. (4) the authors identify a collection of properties that a monitoring service should have from a Fog/Edge point of view, together with a wide overview of the different monitoring models that can be used. However, they do not provide an implementation neither an evaluation. Salman et al. (131) lay out some of the same observations presenting a state-of-the-art review on monitoring Edge computing applications. In their conclusions they state the need for a Fog solution that meets all the monitoring requirements of the Fog/Edge computing paradigm.

Regarding specifically implemented tools, FAST (25) is a Fog-based monitoring tool focused on health applications instead of infrastructure monitoring. In FAST, real-time event detection is distributed throughout the network by splitting the detection task between the Edge devices (e.g., smart phones attached to the user) and the server (e.g., servers in the Cloud).

Wu et al. (145) propose a monitoring approach for Fog systems in a cyber-manufacturing scenario. This proposal presents a fully-developed framework that includes wireless sensor networks, communication protocols, and predictive analytics. Although from different domains, all these approaches are focused on particular application scenarios, and lack the necessary generality to be considered as complete Fog computing monitoring solutions.

PyMon (54) is one of the few fully implemented solutions, where the authors evaluate a lightweight framework designed to monitor elements at the Edge. However, the features are limited, and a more complete solution is needed to meet all the challenges related to Fog monitoring.

One of the gaps we intend to fill with this thesis is providing observability into these kinds of decentralised infrastructures, as the characteristics of their devices and the technologies they are made of present several monitoring challenges. 


\subsubsection{Grid monitoring}

Due to its similarities with the systems we are presenting, Grid monitoring tools are relevant inside our context. The Grid is a decentralised distributed system with the distinctive characteristics of being large at scale and highly geo-distributed (46), just as Fog computing. A set of computing assets, normally personal devices or computers, are shared between organisations in order to complete a certain task through distributed computing. Because of the large scale and the wide geo-distribution characteristics, it is vital to have a view of the different devices in the Grid.

Ganglia (95) is a distributed monitoring system with high scalability and that follows a hierarchical model in which the nodes can be grouped to form a federation of clusters. This approach is suitable for the Grid, in which the different computing resources that need to be monitored are geo-distributed. The Ganglia monitoring agent is called gmond and the entity that provides the coordination of the agents into a federation is gmetad. The latter can communicate with other instances of gmetad to provide aggregated data of the set of federations.

MonALISA (104) is specifically designed to fit an architecture with hundreds of sites, each one with thousands of elements running on them. It is based on JINI and other web services technologies, uses monitoring agents, and incorporates a multi-threaded engine that is able run a set of dynamic services. Those services are registered in a directory that allows other services or clients to discover them.

Astrolabe (136) is not specifically mentioned as a Grid monitoring tool, but we include it here because of its peer-to-peer design, which clearly fits a Grid environment. Astrolabe is able to monitor the state of a collection of resources and organise these into zones. Then aggregations can be calculated for each zone through queries using a SQL syntax. The objective is to avoid a single point of failure as in other solutions, providing a summary of the status of a set of devices.

\subsubsection{Cloud monitoring}

Cloud computing has changed the way software applications are built. Rather than having a local server or personal computer providing a service to a client, the task is offloaded to a pool of powerful servers that is accessed through the Internet. There are several advantages in adopting this type of computing: lower IT costs for companies, 
flexibility, elasticity, better energy efficiency, etc. (72). In the context of Fog computing, the Cloud represents the most powerful level of the infrastructure in computational terms. Although that means that the Cloud is one of the parts of Fog computing, we want to allocate a dedicated subsection to it, as before the appearance of Fog computing, there were many Cloud monitoring solutions with their own characteristics. We will not cover the whole Cloud monitoring landscape, as this has been an important concern of industry and there are many tools available. Instead we will try to provide a representative set of solutions following different monitoring models.

From the academia side, we find GMonE (101), a general-purpose Cloud monitoring approach that covers the different aspects of the Cloud, such as the main Cloud service models (IaaS, PaaS and SaaS), client-side or service provider-side, and virtual or physical side monitoring. It is based on a push model where the GMonE agents push their metrics to a back-end of choice. An important feature in the Cloud is to be able to monitor different technologies through its key metrics. GMonE achieves this through the use of a plugin-based approach in which the agents can be customised to extract different types of metrics.

DARGOS (112) is a decentralized Cloud monitoring tool, designed to efficiently disseminate monitoring information. It is based on an architecture where the agent, called Node Monitor Agent (NMA), extracts metrics for an element located in only one zone, and an entity, called Node Supervisor Agent (NSA), gathers the monitored data from a set of NMA's belonging to one or more zones. These two elements communicate through the Data Distribution Service, a standard data-centric middleware.

At the industry level, Datadog (35) is a commercial Cloud monitoring solution that incorporates a SaaS-based analytics platform. Datadog combines information from servers, platform tools and applications, providing a unified view of the Cloud system. It is one of the most popular monitoring tools in industry, with a high level of flexibility and the possibility of executing the agents as containers that can be easily deployed.

Similarly, CA Application Performance Management (APM) ${ }^{16}$ facilitates the maintenance of the infrastructure by providing data-driven insights and automatically finding anomalies together with the correlations between metrics. Dynatrace (40) commercializes another solution for APM, offering a Cloud-hosted platform-as-a-service (PaaS).

\footnotetext{
${ }^{16}$ https://www.ca.com/us/products/ca-application-performance-management.html
} 
New Relic (103) is also a popular PaaS solution, with a more limited set of features, since only some parts of the monitoring process can be deployed through containers.

Finally, Prometheus (113) is an open-source monitoring and alerting tool that has been widely adopted as a complement for container orchestrators. It follows a pull model via HTTP, where metrics are collected from endpoints that expose them. Although the monitoring agents are not part of the Prometheus framework per se, there is a great variety of them developed by the open-source community.

\subsection{Conclusion: autonomic computing facilitates the man- agement of infrastructures}

Throughout this chapter we have given an overview of the different parts that compose an autonomic computing system. The ability of a distributed system to manage itself, can take the burden off the users and operate itself in a more efficient way. This is specially important in an environment such as IoT with numerous elements using different technologies each. As described in the literature, there are many ways to build the knowledge needed by autonomous computing systems. We believe this can be achieved by monitoring the many parts of a distributed system and using artificial intelligence to establish correlations between metrics and events in the system. The main reason behind the choice of artificial intelligence techniques is that systems nowadays are far too complex to be completely analyzed and understood by expert users. Besides, systems evolve all the time and previously unseen events can occur in the infrastructure. Using AI together with feedback from the user, the autonomic computing system will be able to adapt to this new situation and react accordingly. Users can provide valuable feedback into the system, which can still keep its autonomous capabilities. The degree of autonomy can also vary. Depending on the criticality of the decision or its impact, users can let the autonomous system make the decisions directly or include a human filter. These ideas will constitute the basis of the tools developed throughout this thesis to control several technologies inside the decentralised IoT landscape, which will be presented in the following chapters. 


\section{Part III}

\section{Contributions: Managing, optimising and monitoring the IoT technological stack}





\section{Chapter 4}

\section{General Methodology}

So far we have laid out the context in which our work is developed. We have talked about a new type of data processing applications that rely on the information generated by sensors or things that register events in our everyday lives. We also described a new type of distributed system, called Fog computing, that was designed to meet many of the requirements of these kinds of applications and that involves several technologies, such as microservice architectures and Big Data analytics platforms.

The challenges that we will address in this thesis derive from the management of these kinds of systems. Inspired by the autonomic computing idea, we design a framework that uses artificial intelligence models to support the user in this task. We can choose different levels of autonomy, as discussed in Section 3.1.5. Instead of relying on a completely autonomic system, we want to include the user in the process because of two main reasons. Firstly, by using models that are explainable, we can help the user to understand the factors affecting the performance of the system. Secondly, by adding a human-in-the-loop, the user can provide feedback and expert knowledge that can be used to correct any mistakes in the model and improve it. The objective of our thesis is to evaluate if this framework can improve the use and maintenance of these complex infrastructures.

\subsection{Artificial Intelligence for autonomic computing}

We mentioned before that one of the foundations for autonomic computing was the MAPE-K model, standing for monitor, analyse, plan, execute and knowledge. Each 
of these functions can be implemented in different ways. In fact, the monitor part can be done by leveraging any of the tools currently available in the market, as we detailed in Section 3.2. Same with the analysis, as many of these monitoring tools provide ways to create insights from the metrics in the form of dashboards and other visualisation techniques. The planning, execution and knowledge parts are probably the most complex ones.

The knowledge is the pillar in which the planning and resulting execution are based on. This is an intricate process, specially if we consider that the monitored information might be very large for a distributed system with numerous monitored elements and data coming from different monitoring solutions. We have several options when implementing the intelligence that will guide the user with the management of the infrastructure. For example, a repository of rules set by an expert can be used to suggest the right actions depending on the observed metrics. This is far from ideal as systems change very quickly, specially in environments such as microservices. That is why we believe artificial intelligence models are better suited for this task, since they will learn the rules directly from the metrics and events that happen in the system. These intelligent models can be later used to propose plans to the user.

To create the models, we follow a basic data science pipeline, such as the one illustrated in Figure 4.1. Each of the steps will have a different approach depending on the type of technology we want to manage, but the general methodology will be the same. At the end, we will have a model that will be used by the framework to build the appropriate plans necessary to achieve self-optimisation and self-healing.

\subsubsection{Monitoring}

The first step is to gather the data that will be used to model the performance of these complex systems. We will not expand on this part as we have already talked about it through the Section 3.2 of the thesis. Using monitoring tools we can capture the state of the different elements in the form of metrics and complete the first step in MAPE-K. Several systems, such as Prometheus or GMonE, provide backends in which this information is stored to be queried in the future. We will use these back-ends as our source of data to produce the inputs of the AI models. 


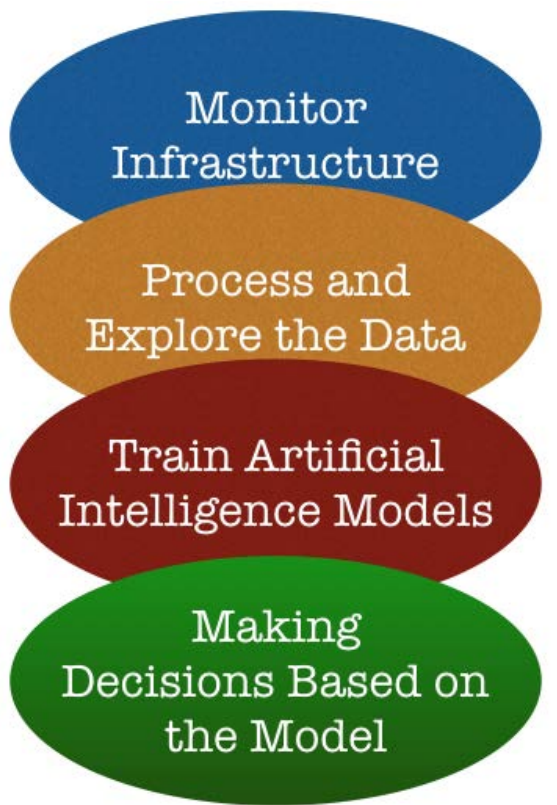

Figure 4.1: The data science pipeline used to build AI models for infrastructure management

\subsubsection{Process and integrate the data}

Distributed systems have complex architectures with different layers that need to be monitored. Many monitoring tools provide an all-in-one solution and are able to extract the information from any of these layers. Still, if we use different monitoring tools we will need to integrate all the different sources of information. In the context of artificial intelligence, if we are going to use machine learning models, we also need to specify the variable of interest that needs to be predicted and that will guide the learning process. For instance, we might want to build a model able to predict from metrics in real time if an event is going to happen in the system. In this case, we will have a classification problem. Another outcome can be predicting the time it will take to execute a job in the system, which will be a regression problem. In addition, we need to preprocess the data, ensure its validity and extract features from it. A preliminary analysis can also be presented to the user by means of descriptive statistics or data visualisation. 


\subsubsection{Train artificial intelligence models}

The features extracted from the data are fed into the machine learning model of choice in the next step. We will ideally use interpretable models that can be understood by the user. Certainly, if we are going to suggest decisions involving critical infrastructures, the models should be interpretable in order to understand the reasons and increase the confidence of the user in the system. Additionally, exposing different correlations between input and output variables can help to understand these complex systems. Finally, the user will be able to provide feedback based on this interpretability and understanding of the systems. Doran et al. (39) provide a good definition of the different types of AI systems depending on their interpretability:

- Opaque systems: The relation between inputs and outputs is not shown to the user or is not comprehensible. We will avoid these kinds of models as we want the user to interact with the autonomous system and understand the suggested actions. Some examples include proprietary AI modules or deep learning methods.

- Interpretable systems: The user understands the choices of the system and an explanation is given, mapping the inputs to the outputs. For example, in regression models such as linear regression or SVM's, the weights of each feature represent their importance in the final outcome.

- Comprehensible systems: They produce user-understandable symbols together with the prediction. These symbols can be words, visualisations or any kind of notation that facilitates the interpretation of the result. An example would be a decision tree in which the user can traverse the tree based on the values of the inputs.

As we will see in the following chapters, our models are both interpretable and comprehensible, enabling the possible integration of these models with a user-friendly decision support system.

\subsubsection{Making decisions based on the outputs of the model}

The final output of these models will be used to suggest an action to the user, such as configuring the system in a given way or executing a contingency plan to solve 
an anomaly. This could involve some logic that combines the raw prediction of the AI model together with other inputs to provide a final decision. As the models are interpretable and can be understood by humans, we are going to use a human-in-theloop approach to improve our framework, such as the one depicted in Figure 4.2. It has been used in other fields, such as health informatics (63), and it is sometimes called interactive machine learning. It includes the user in the learning process, as some tasks are difficult to automatise completely when they need some expert knowledge. An example would be the occurrence of a previously unseen event in which the expert can apply knowledge from other situations or information that has not been captured by the autonomic computing system. In this approach, the user is involved in the process of creating and enriching the data used by the AI pipeline together with the tuning and testing of its models. This has the advantages of both improving the accuracy and correcting any mistakes on the predictions. An example is a face recognition algorithm that asks the user for confirmation on its prediction if the confidence is below a certain threshold, or directly asks to label manually the photo. We will use this technique explicitly in our root cause analysis model for microservices.

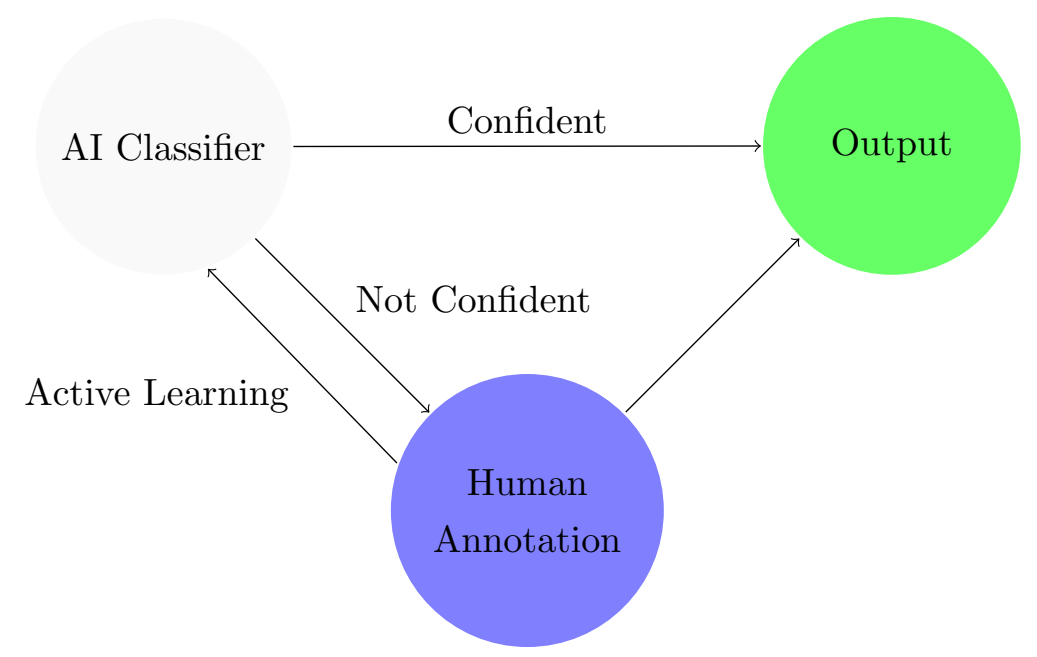

Figure 4.2: Human in the loop

\footnotetext{
${ }^{17}$ https://www.figure-eight.com/resources/human-in-the-loop/
} 


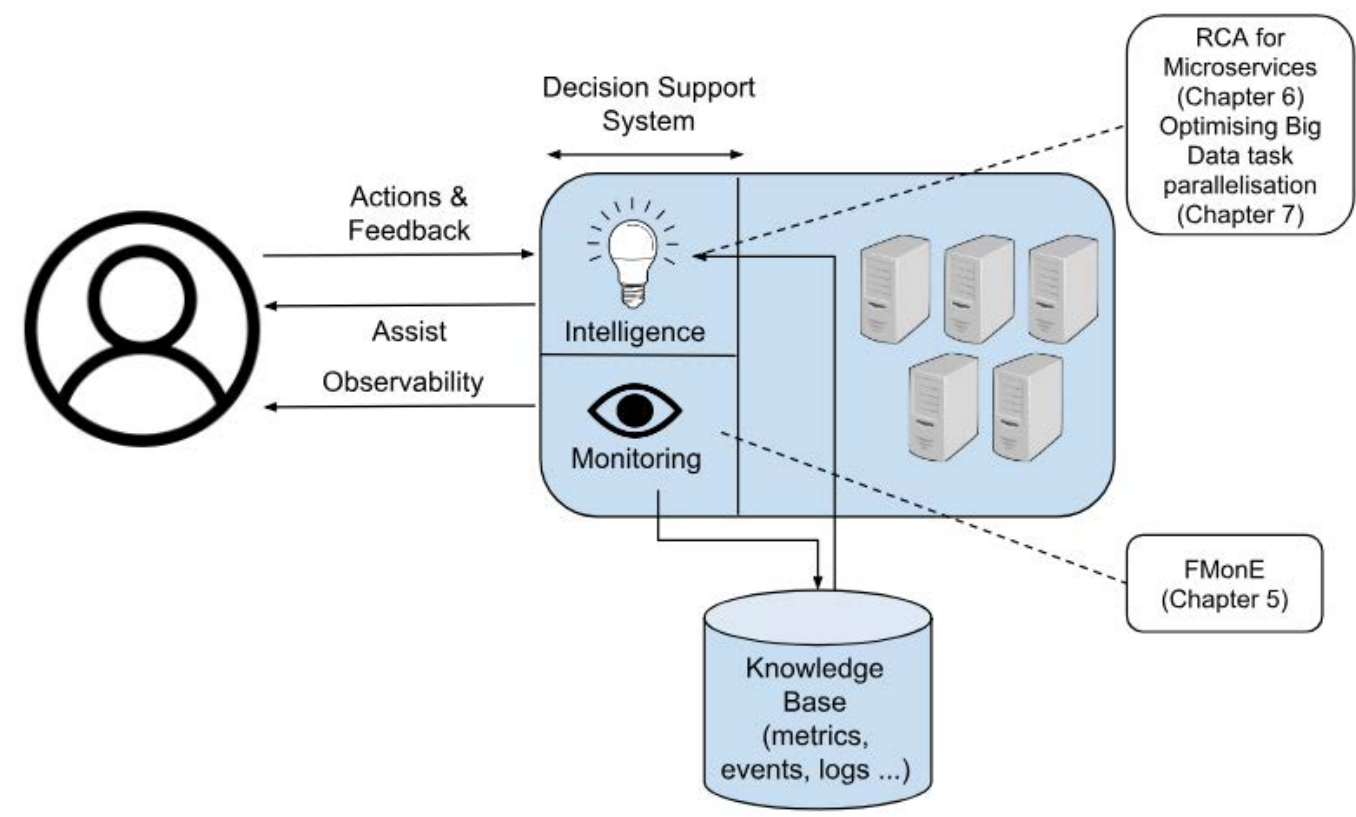

Figure 4.3: The framework used to manage and provide observability of the infrastructure. Our contributions are detailed in Chapter 5 for the monitoring part and in Chapter 6 and 7 for the intelligence part of the system

\subsubsection{A decision support system prototype for the Fog}

By combining the ideas of autonomic computing, artificial intelligence and a human-inthe-loop approach, we build a framework that reassembles a decision support system (DSS) as the one shown in Figure 4.3. In this prototype of DSS for IoT technologies, our monitoring tools (Chapter 5) and developed models (Chapter 6 and 7) will provide the core features of monitoring and intelligence. The benefits for the user are improved observability and assistance in the decision making. Based on the insights produced, the user can give feedback to the model or apply the suggested actions directly in the system.

\subsection{Conclusions: creating intelligence by monitoring the system}

In this chapter we have introduced our methodology to manage the distributed systems that are core to the IoT. It is based on a framework that imitates a DSS. It provides 
observability into the state of the infrastructure as one of its features. The implementation of the intelligent part of the DSS is inspired by the MAPE-K model, which is the cornerstone of the autonomic computing idea. We discussed how the knowledge part can be automatically learned through AI techniques that use the information coming from monitoring the target distributed infrastructure. Additionally, the AI algorithms are interpretable and the users can be included in the workflow, by allowing them to change and tune the resulting models.

In the following chapters we will present our work, implementing some of the models and tools needed to support the decision making process over an IoT infrastructure and its associated technologies. We will start with FMonE, a tool that provides observability for a decentralised infrastructure such as Fog. We will follow with a graph-based root cause analysis model for microservices that identifies the source of a failure in a containerised architecture. Then, it can be used to propose a contingency plan or execute it automatically, meeting one of the self-healing requirements for autonomic computing. Finally, and following the self-optimisation paradigm of autonomic computing, we implement a model that is able to recommend the parameters needed to optimise the task parallelism of a Big Data platform, which can be considered as the highest level of the Fog hierarchy. 


\title{
Chapter 5
}

\section{FMonE: An effective monitoring tool at the edge}

\begin{abstract}
Monitoring has always been a key element on ensuring the performance of complex distributed systems, being a first step to control Quality of Service, detect anomalies or make decisions about resource allocation and job scheduling, to name a few. We have previously introduced the Fog computing paradigm, which was designed as a distributed infrastructure that could support the particular requirements of IoT applications. Some of the differences between this approach and more traditional architectures, like cloud or HPC, are that these devices have low computing power, unstable connectivity and are geo-distributed or even mobile. This new type of complex environment poses new challenges in the monitoring field that have not yet been considered. In this chapter, we present a study of the requirements that a Fog monitoring tool should meet based on a series of motivating scenarios drawn from the literature. We also detail the design of a monitoring tool named FMonE, meant to implement the observability part of our DSS. FMonE is a light-weight, installation-free and user-adjustable monitoring framework, specially designed to monitor a Fog system. It relies on a container orchestration system to build monitoring pipelines that adapt to the distinct features of a Fog infrastructure. We evaluate FMonE by simulating a Fog environment in the Grid'5000 testbed and we demonstrate that it fulfills the requirements we previously enumerated. The content of this chapter is based on the article "FMonE: A Flexible Monitoring Solution at the Edge" (22).
\end{abstract}




\subsection{Motivating scenarios}

Cloud is a well-known, established technology with a clear business model behind it (93). Cloud architectures support general-purpose services at different levels, relying on centralized data centers and infrastructures. Fog is expected to provide different services and applications at the edge of the network, to support a specific commercial activity, by means of a decentralized infrastructure. Similarly to the Cloud, Fog services and applications need to be monitored in order to provide observability and means of control to the user.

In this section, we present two motivating scenarios by broadening the ideas presented in the literature (18): a telecommunication company that needs to keep track of the usage of all their devices at the edge and a provider of a Platform as a Service (PaaS) for multiplayer games that needs to monitor the different elements of the platform. These scenarios will illustrate the challenges that arise when the monitoring workflow has to be changed from a cloud environment (centralised, homogeneous, stable) to a fog environment (decentralised, heterogeneous, unstable).

\subsubsection{Monitoring an edge infrastructure}

Telecommunication companies, also called telcos, are one of the key actors in the Fog computing paradigm (140). The Fog allows them to take advantage of their geographically distributed infrastructure, made of small smart devices like gateways, smart phones or any other kind of device with storage and computing power. This infrastructure can be used as the bare metal that hosts services in closely delimited regions, such as cities or districts, placing them closer to where they will actually be used.

In this sense, telcos should be able to properly manage their resources, to enable Infrastructure as a Service (IaaS) at the edge of the network. All this requires to monitor the different running devices, to make decisions that are analogous to those needed in a data center: scheduling, resource allocation, QoS or SLAs, among others. But in this case, the resources are not traditional nodes running in a data center, but small to medium smart devices, with different hardware specifications. There can be thousands of these smart nodes in every geographical region, which must be monitored despite their differences. 
Monitoring such an infrastructure poses several challenges. Firstly, nodes may join and leave the network frequently (e.g. consider a mobile device that can leave the carrier's coverage area, shutdown or fail). The monitoring services should be able to handle this type of behavior. Secondly, job scheduling, resource provisioning and allocation mechanisms require monitoring metrics with very low and predictable latency to make fast decisions. However, the round-trip time between the central data center and the edge devices in a geographically distributed infrastructure could be long. This creates the need for keeping the monitoring data at the edge, following the same paradigm as fog applications, something that is not easily achieved with current monitoring solutions. Additionally, and as it will be shown later in our experiments, the constrained bandwidth of devices at the edge is another important factor in the monitoring process. The monitored information sent outside the local network should be kept to a minimum, in order to leave the available bandwidth to processes that need to use it intensively. Finally, if monitoring metrics are going to be kept at the edge, we need to consider how they are going to be ingested and stored by the devices (e.g. key-value stores, time series database, message queue, etc). This depends, firstly, on the amount of measurements, and secondly, on the computation and storage capacity of the devices hosting these back-ends. Consequently, we should be able to change the metrics back-end in a flexible way.

In summary, Fog IaaS needs to be monitored at the same level as its cloud counterpart. However, the particular characteristics of the devices in this infrastructure make it necessary to incorporate new functionalities to Fog monitoring tools. These functionalities are not present in previous centralized and more homogeneous environments.

\subsubsection{Monitoring service applications at the edge}

Mobile online games could be one of the industries that most benefit from Fog computing. It would reduce latency problems for users, by bringing the game endpoint closer to them, as well as including an additional location context that is required by many augmented reality applications (34). Within this field there are companies that offer a Platform as a Sevice (PaaS) for game developers to abstract things like scaling, player management, user segmentation and many complex multiplayer features 18 . It also assures game developers that their games are going to run within some QoS and SLA's

\footnotetext{
${ }^{18}$ https://www.gamesparks.com/
} 
that PaaS has to comply with. If we imagine this PaaS running in a decentralized Fog environment, there are some challenges to be considered in terms of monitoring.

First, there is a disparity of technologies that will form the backbone of this platform, such as databases, messages queues or data processing frameworks. Ensuring that each of these pieces are working properly means different types of metrics depending on the technology that is being monitored. Secondly, we expect high computing and networking demands in these kinds of applications. Much of the data has to be moved from the server to the gaming clients in a multiplayer environment, thus the monitoring system should not clog the network connection or stall the computing power of Fog nodes, especially when their resources are limited. Finally, we may need to look more closely at the problems that could arise in a particular region. This means that we should be able to deploy monitoring agents on a subset of problematic devices e.g. all the devices corresponding to a user segment.

From this use case we can draw the conclusion that an already complex system like PaaS can be really difficult to monitor if we add an extra layer of complexity like Fog computing. A monitoring tool for such an environment should facilitate this process to the user as much as possible.

\subsection{Requirements of a Fog monitoring system}

From the previous scenarios we can extract the following requirements for a Fog monitoring tool:

- Installation-free (R1). As previously mentioned, there is a clear need of a dynamic, heterogeneous infrastructure, such as the ones from telcos. The node heterogeneity would hinder an installation process with different dependencies based on their architecture. In addition, nodes can join the network at any time, creating the need for installation-free monitors that need to be up and running as soon as possible.

- Aggregation/filtering of metrics (R2). Fog applications have an implicit aggregation workflow that starts from the edge and ends at the central cloud, as seen in Figure 5.1. We believe monitoring of such an infrastructure should be done 
the same way. Fine grained data can be kept at the edge for regional data analysis, and aggregated information can be sent to the cloud for central reporting or visualization purposes. For example, a telco could have a dashboard that shows the CPU usage by region, overlapped with a map showing the location of its fog computing assets at the time. The telco can navigate up and down the data to have a better control of the infrastructure and its metrics. In addition, users should be able to define a criteria for non-important data, that can be filtered out to not create any additional traffic or storage burden.

- Flexible back-ends (R3). There are several implications that must be considered when storing monitoring metrics. Some nodes closer to the edge are more unreliable, in the sense that they can disappear from the network at any moment. Therefore, metrics should be stored on more reliable nodes. Also the volume and speed of the monitoring process can change depending on what elements are being monitored, and from how many nodes. For example, for a small amount of monitored components, a MySQL database might be enough. On the other hand, for an intensive log monitoring task on a large geographical area of nodes, we might need a more scalable database, like Cassandra. This requires a flexible back-end in both type and location for the monitored data. Additionally, there may be several back-ends for the same data at more than one of the levels presented in Figure 5.1 .

- Elasticity (R4). Nodes are being continuously added and removed from the network. For example, users can lease their mobile devices as a computing asset (137) for telcos. A fog monitoring solution should be able to detect any node that becomes part of the infrastructure and start monitoring the device and its components as soon as possible. Also, the whole monitoring process should not be hindered by the disappearance of any node in the infrastructure.

- Resilience (R5). The nodes in the fog can be faulty, specially considering that they can have limited resources. If a monitoring agent fails, the system should be able to relaunch it. Also, if the host of an important point of the monitoring workflow (e.g. the back-end) disappears, we should be able to reallocate that point to another host nearby. 
- Geo-aware (R6). A fog platform has an inherent geo-distributed structure. Nodes are divided in regions depending on their location, which leads to better connectivity between them through local connections and fewer hops across the network. This creates the need of deploying a set of monitoring agents and their back-ends on the same region, in order to leverage these local connections and avoid unnecessary latency in the monitoring workflow. It can also be used to get more detailed data of problematic regions where we need to zoom into a specific technology that is only found on that region. An example of this workflow would be to deploy specialized monitoring agents with a specific plugin in one region hosting a particular set of technologies (e.g. an agent that monitors the number of messages ingested by a Kafka queue in one region).

- Plugin-based monitoring (R7). Different technologies run together in this kind of infrastructure and we might need to monitor them at different levels. For instance, we might want to monitor a Mongo database, a Docker container and a Kafka queue which are part of the chat system of the above-mentioned online gaming PaaS, taking into account that each one will need different kinds of metrics. The deployment agent should have plugins that enable the extraction of these diverse metrics by specifying the types of component that need to be monitored.

- Non-intrusive (R8). Some devices at the edge infrastructure are resource-poor. Consequently, the monitoring process should interfere with the normal functioning of these already constrained devices as less as possible. Especially, the amount of monitored information sent over the network must be kept to a minimum (as bandwidth may be limited). This is specially true when we have demanding applications, like online gaming, running on the nodes.

- Hardware and operating system agnostic (R9). A fog infrastructure can consist of normal nodes, mobile phones, gateways or any other kind of devices that can provide some kind of computation power. The monitoring tool should be able to run on these broad spectrum of technologies, independently of the operating system or hardware underneath. 


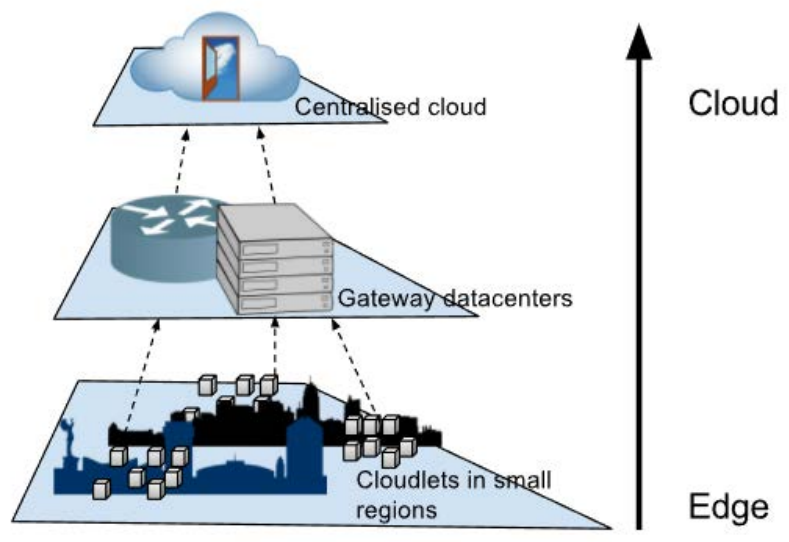

Figure 5.1: A Fog infrastructure starts at the edge of the network, where the data is generated at the IoT layer. Small data centers provide computation and storage close to the applications. If data needs to be sent to the central cloud or intermediate gateways, it is aggregated in a bottom-up fashion.

\subsection{FMonE design: Tackling the challenges}

In this section we explain the design principles of FMonE, a monitoring tool created to satisfy the requirements of Section 5.2. By leveraging existing technologies, FMonE facilitates to the user the deployment of the monitoring agents across the Fog infrastructure. The main advantage of its plugin-based design is to enable the creation of monitoring pipelines that are adapted to the above-mentioned Fog particularities.

\subsubsection{Architecture of FMonE}

FMonE is designed as a framework that includes the monitoring agents together with the management of those agents and their back-ends. The general architecture of the system is depicted in Figure 5.2. We explain here each of the parts.

- FMonE: This is the general framework that coordinates the monitoring process across the whole fog infrastructure. It is a Python framework that communicates with Marathon 19 a container orchestrator able to coordinate and maintain across the fog devices the monitoring agents and the back-ends for the metrics. Both the monitoring agents and the back-ends are implemented as Docker containers, which can be easily deployed in any kind of node without any installation process and

\footnotetext{
${ }^{19}$ https://mesosphere.github.io/marathon/ (last accessed Feb 2018)
} 


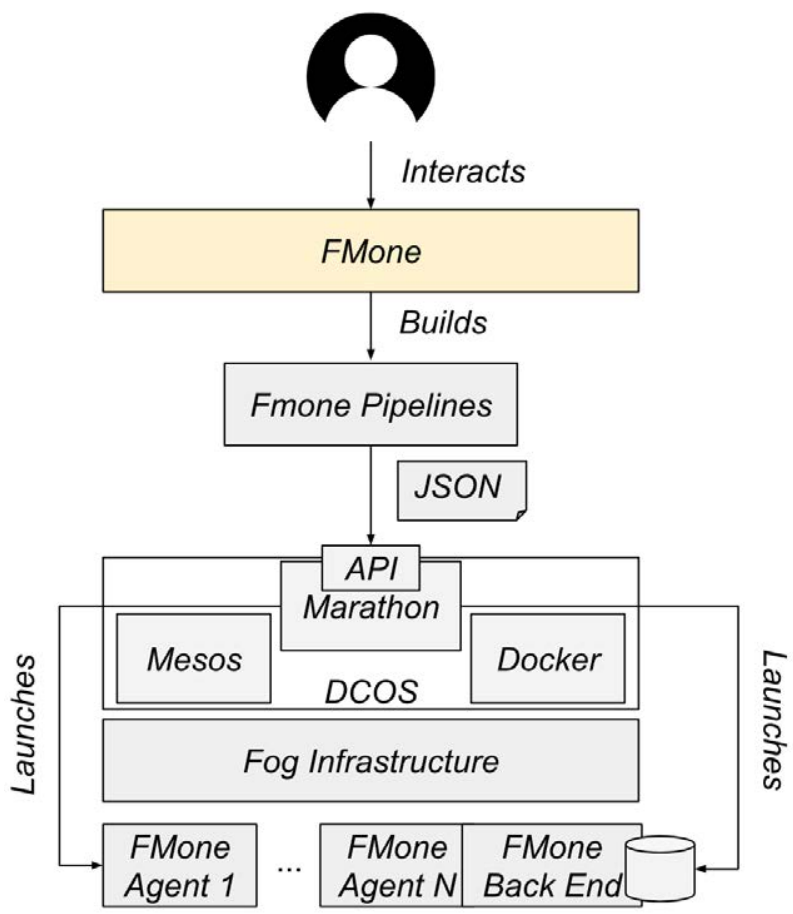

Figure 5.2: The general architecture that supports FMonE. The user interacts with the FMonE framework (through an UI or command line not yet implemented) which acts as a translator between the user needs and a Marathon deployment plan. When the user has built the monitoring workflow this is translated into an FMonE pipeline, which in turn is passed as a JSON file to the Marathon API. Marathon is then responsible for launching the monitoring agents and the back-ends throughout the infrastructure

independently of the platform, fulfilling requirements $\mathbf{R} \mathbf{1}$ and $\mathbf{R 9}$. The Marathon container orchestrator with which FMonE communicates, forms part of DCOS 20 a software package that can be easily installed on all nodes. The architecture of DCOS is shown in Figure 5.3. More information is available in their website. Besides Marathon, it also deploys the Docker engine and Apache Mesos (62), which acts as a robust cluster manager that can scale to thousands of nodes. This combination of technologies will facilitate to FMonE the deployment of its different agents and back-ends. Note that any other container orchestrator could be used instead of Marathon. Ideally, this orchestrator should be lightweight in order to work with guarantees on a fog infrastructure. In this paper we have

\footnotetext{
${ }^{20}$ https://dcos.io/docs/latest/overview/what-is-dcos/ (last accessed Feb 2018)
} 
chosen DCOS, because of its ease of deployment and several features that allow us to meet the following requirements:

- High availability: Marathon achieves high availability through Zookeeper (69). This means that there are several Marathon instances available and, in case that the current leading instance fails, a new leader will be elected, achieving $100 \%$ uptime. Additionally, it takes care of relaunching any Docker container that fails or relocating it if its current machine fails. This enables requirement $\mathbf{R 5}$.

- Constraints: It allows us to specify a criteria to control in which nodes the FMonE containers should be executed. An example would be to assign a label to the fog nodes depending on the region they belong to and then constraining the deployment of a set of containers to a specific region. As long as the different resources in the fog infrastructure are correctly labeled, it will allow us to have a geo-aware control of the fog infrastructure, by using FMonE to launch monitoring agents in specific regions only. This fulfills requirement $\mathbf{R 6}$.

- Adding nodes: Through the above mentioned constraints, we can also define that in each host an FMonE agent container must be active. As soon as a node joins the system, the framework will start monitoring it without the operator intervention. Therefore we can meet requirement R4. Remember that constraints are part of the Marathon API ${ }^{21}$ and that FMonE uses them to achieve these features.

- Pipeline: It represents a workflow of FMonE agents that can optionally communicate with each other and/or dump their metrics to one or more back-ends. Users can define their own pipelines. For the purpose of this paper, we have defined three pipelines, as we will see in the experiments section (Section 5.4). Nonetheless, an interactive layer such as a dashboard or a console could be added to FMonE, allowing the user to examine the different regions, the devices assigned to them and facilitating the definition of new pipelines. Once the pipeline has been defined, it will be translated by FMonE into a Marathon application groun 22 , JSON file

\footnotetext{
${ }^{21}$ https://mesosphere.github.io/marathon/docs/constraints.html (last accessed Feb 2018)

${ }^{22}$ https://mesosphere.github.io/marathon/docs/application-groups.html
} 
that establishes an order for the containers to be deployed. Figure 5.5 includes a description of the conceptual parts that constitute the pipeline concept. Each pipeline takes care of one or more regions of the fog, as the geo-distribution of its devices is one of its distinctive characteristics. In this way users can build different pipelines with different configurations for its monitoring agents, depending on the physical location of the devices. It also facilitates placing them in problematic regions or near their back-ends. A pipeline can have one or more back-ends, which are hosted in one of the regions. Monitoring agents in a pipeline can store their metrics in one of these back-ends, which can be an existing one (previously launched in another pipeline) or a new one, which is created. In each of the regions covered by the pipeline, one or more FMonE agents are launched. For the FMonE agents a set of InPlugin, MidPlugin and OutPlugin has to be chosen. Details about the plugins will be provided later in this same section. Figure 5.4 shows an example of a pipeline. As explained in Figure 5.5, a pipeline takes care of monitoring several regions, in this case region A, B and C. Each region has a different number of FMonE agents, whose plugins can be chosen by the user. In region $\mathrm{A}$ all the metrics are dumped into an existing back-end 2 in Region D. Region $\mathrm{B}$ has 4 agents, three of which perform monitoring tasks and another one aggregates the metrics extracted by the other three. The aggregation is stored in back-end 2. The same pattern is used in region $\mathrm{C}$, but this time we store the full detail of the metrics in back-end 1, hosted in the same region, while another agent aggregates the metrics and store them in back-end 2. This means that metrics can be stored in several back-ends at different granularities. All these data can be queried from the intelligence in the central cloud or by actors at the edge. As previously mentioned, these pipelines enable to change the monitoring workflow depending on the user needs, aggregating metrics and storing them at different levels (e.g. gateways or IoT devices with storage), fulfilling requirement R2.

- Flexible back-ends: Users should be able to choose a back-end for their metrics depending on several factors, like the number of elements to be monitored or the machine in which this back-end is going to be installed. It is evident that if a large amount of metrics needs to be stored, more scalable technologies like Kafka should be used for the ingestion. On the other hand, if we are going to store 


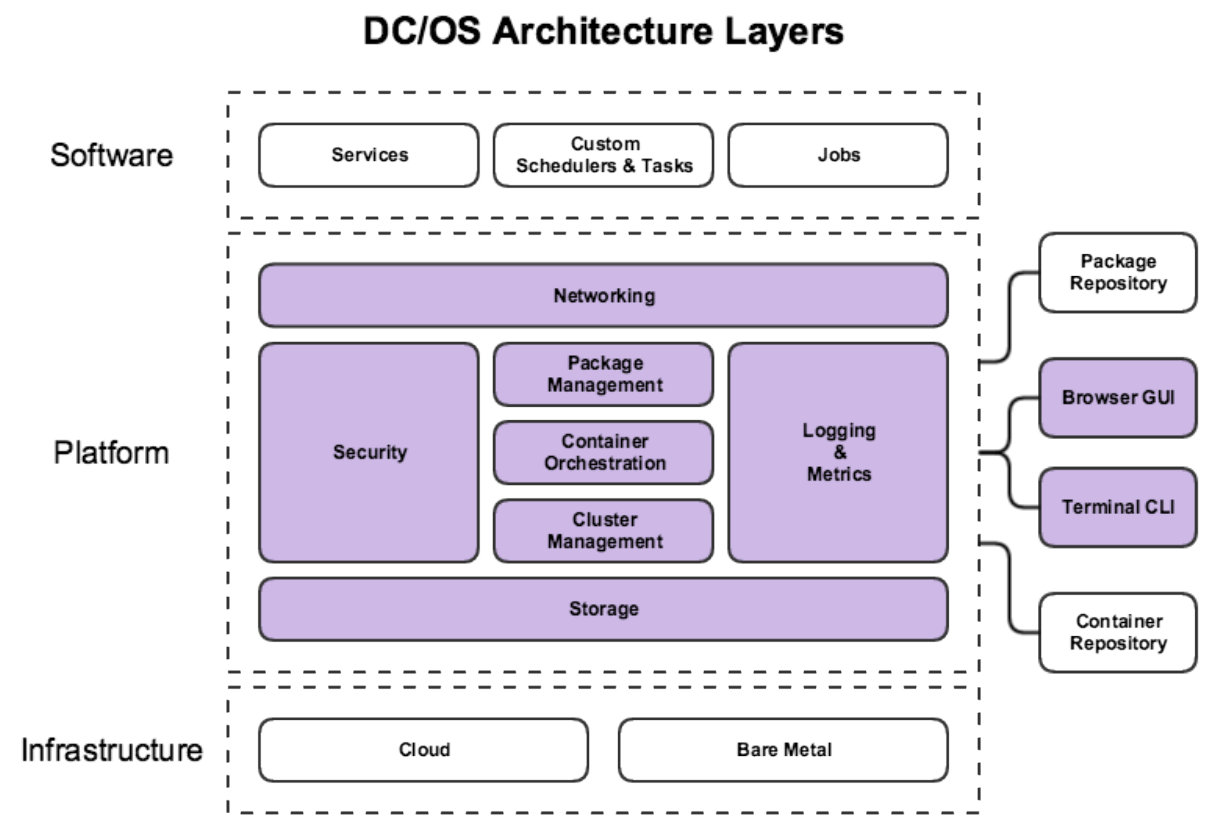

Figure 5.3: The DCOS architecture 23

fewer metrics on a low resource device, we could use lightweight databases like sqlite, which has already been used in some IoT prototypes (5). In any case and since a fog environment is very diverse, this should be flexible and not limited to one technology. It is not mandatory for a pipeline to have a back-end, since an FMonE agent can always take advantage of an existing one. There is also the possibility of creating one back-end or more for that pipeline. These backends are also implemented as Docker containers and the most popular database vendors already provide them. The users can choose the one that best fits their use cases, satisfying requirement $\mathbf{R} 3$.

- FMonE agent: This is the Docker container responsible for monitoring each of the devices. We divide a monitoring job into three fundamental tasks: collect, filter and publish metrics. Their responsibilities are divided into three plugins:

- Inplugin: The plugin extracts the monitoring information from a component of the system every $x_{\text {collect }}$ seconds. This set of metrics is kept in memory before publishing it to the back-end. Examples of these metrics are the CPU load, the stats of a Docker container, or the number of messages received per 
second in an MQ queue. We have defined a series of plugins that the users can use, allowing them to create new ones on their own by implementing a simple interface. This versatility to monitor different technologies answers to requirement $\mathbf{R 7}$.

- Midplugin: Here the user can define a custom function that filters or aggregates the set of metrics that the agent keeps in memory. This function is applied before publishing the values. Aggregating and filtering metrics can reduce traffic and fulfills requirement $\mathbf{R 8}$, for example, publish CPU load values that are above a certain threshold. A more complex logic can be used, from simple aggregates, such as averaging metrics for a time window, to a machine learning classifier that decides whether or not a metric should be published.

- Outplugin: It dumps all the filtered data by the Midplugin to a back-end every $x_{\text {publish }}$ seconds. This back-end can be any kind of storage as long as it is supported by an outplugin implementation. A file, a document database such as MongoDB or a key-value store like Cassandra are some examples of back-ends. Another target can be a message queue, from which another FMonE agent can pull, aggregate/filter the metrics and push them to a

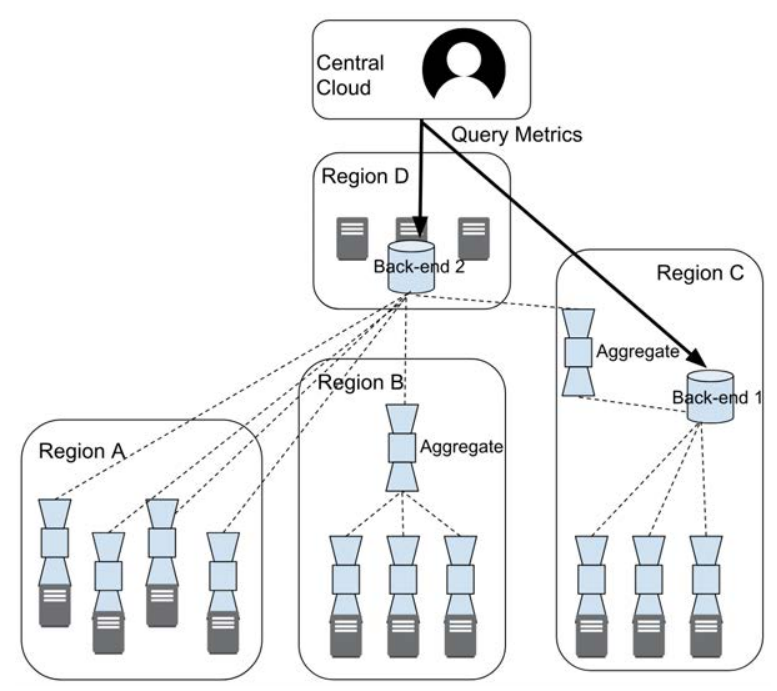

Figure 5.4: An example of an FMonE pipeline that takes care of three different regions using two back-ends. Users can query the metrics stored in any of the different back-ends. 
different location. This is part of the requirement $\mathbf{R 3}$.

Figure 5.5 includes a description of the conceptual parts that put together the monitoring process.

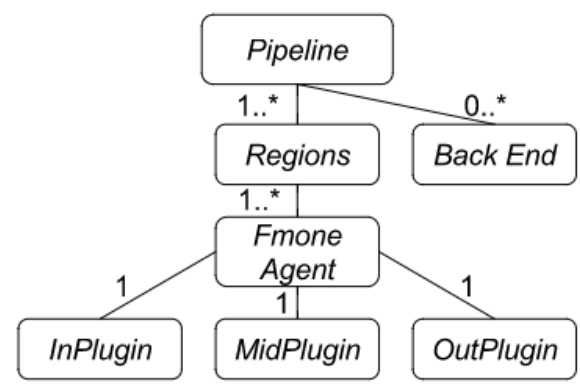

Figure 5.5: The different concepts that form a monitoring pipeline. A pipeline takes care of one or more regions of the Fog infrastructure. Additionally it may have several backends. A set of one or more FMonE agents is launched in each region. These agents have three components to gather the metrics: InPlugin to collect data, Midplugin to process it and OutPlugin to publish it.

\subsubsection{Using FMonE}

When users want to launch an FMonE pipeline, they have to specify the monitoring workflow throughout the Fog infrastructure. First, they choose a set of regions that the pipeline is going to take care of. Afterwards, they have to specify for each of the regions the $x_{\text {collect }}$ and $x_{\text {publish }}$ intervals together with the Inplugin, Midplugin and Outplugin that they want to use for the FMonE monitors. Moreover, additional parameters can be passed to the plugins if it is needed. For example, if we want to push metrics to a Kafka queue we need to give as a parameter the name of the Kafka broker and the topic we want to publish to. This process enables the extraction of metrics at different levels of the system, their processing and their storage in the chosen back-ends in a flexible way. Lastly, the user can optionally provide one or more back-end types (MongoDB, Cassandra, InfluxDB, etc.) that will be created as part of the pipeline. The back-ends do not have to be in the same region as the agents, although it is recommended because of the previously mentioned latency between locations. This user specification will be translated into a container deployment plan inside the Fog infrastructure, in our case, powered by Marathon. As a final summary of how these 

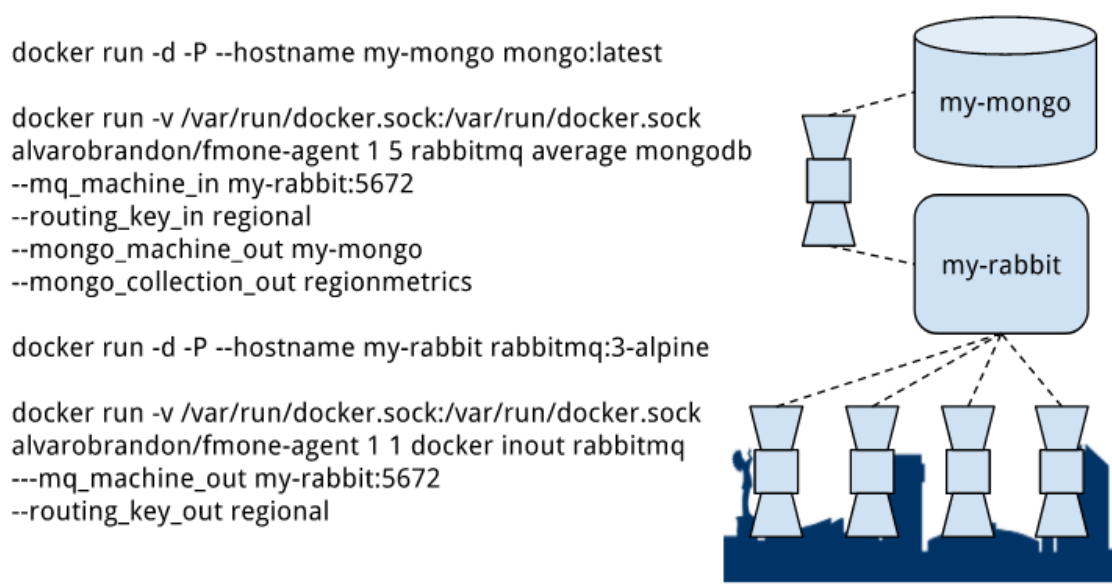

Figure 5.6: Several Docker containers are involved in an FMonE pipeline. To extract the metrics, the Docker image for FMonE is started in each host, with docker as the Inplugin parameter, inout for the MidPlugin and rabbitmq as the Outplugin. The RabbitMQ container will also be created as part of the pipeline and its hostname used as a parameter for the previous outplugin. The aggregation will be performed by a different FMonE agent, which will pull the metrics with its corresponding rabbitmq Inplugin, average them with the average Midplugin and store them in a database with the mongodb Outplugin. The pipeline will be completed with the creation of a containerized MongoDB back-end. Marathon and Mesos are used to start these containers in the desired regions

pipelines are translated, we detail an example in which the user wants to monitor all of the container metrics in a single region, send them through a RabbitMQ message queue to another FMonE agent that averages the metrics for the last 5 seconds and finally stores them into a MongoDB. The example is depicted in Figure 5.6. Note that all these Docker commands are automatically executed by Marathon, the container orchestrator tool, while FMonE takes responsibility for building the deployment plan needed by Marathon for each monitoring pipeline.

\subsection{Evaluation}

This section evaluates FMonE, demonstrating that the prototype we have built meets the above mentioned requirements (see Section 5.2). A prototype of the agent can be found on Docker Hub 24 . We plan to upload in the future the DCOS framework able to coordinate the deployment of the FMonE pipelines.

\footnotetext{
${ }^{24}$ https://hub.docker.com/r/alvarobrandon/fmone-agent/
} 
To set up the evaluation environment, we have considered the reference scenarios shown in Section 5.1. We envision a Fog architecture where an organization has resources in different geographical regions. These resources can be of any nature: devices, servers or any software component that resides in the Fog and fuels the applications from users on different locations. Since the regions are distant from each other, there are bandwidth and latency restrictions between them. As previously shown in Figure 5.1, a Fog architecture assembles a tree as a hierarchical structure. A central region is located at the highest level and all the other regions communicate with it, either to pull data that applications at the edge may need, or to push data needed at the central location. From the root of this tree, the organization needs to monitor and makes decisions about its Fog infrastructure through the DSS that we envisioned in Chapter 4. For example, thanks to the monitoring agents, we can detect overloaded Fog devices in a given region or a node that went down. There are several issues that arise when monitoring this type of environment. The first one is whether sending all this monitoring information to a central location affects application performance (Section 5.4.2). We hypothesize that it does, since at the edge the connectivity resources such as available bandwidth are limited. The flexible monitoring pipelines that FMonE provides, are expected to alleviate that effect. Overhead and resource usage are also important characteristics, since we do not want the monitoring process to interfere with the host performance (Section 5.4.3). We also need to know how fast we can deploy a monitoring agent in an ever-changing infrastructure where elements are constantly joining and leaving the network (Section 5.4.4). An autonomous and resilient monitoring system is also needed in such an unstable scenario (Section 5.4.5). Finally, we provide a qualitative evaluation of FMonE, comparing it with other tools and research work in the field (Section 5.4.6).

\subsubsection{Experiment set-up}

We simulate a Fog scenario with virtual machines hosted in the Grid5000 testbed (14) to evaluate FMonE at large scale. Grid5000 is a testbed that provides access to a large amount of computing resources. It is highly customizable and offers a wide range of tools for reproducible experiments. We use Vagrant together with the vagrant-g51 25 plugin specific to this testbed to provision the VMs. Grid5000 has nine sites available

\footnotetext{
${ }^{25}$ https://github.com/msimonin/vagrant-g5k
} 
with several machines each. We launched $78 \mathrm{VMs}$ in the Rennes site, with 4 cores and $10 \mathrm{~GB}$ of RAM and we configured the testbed infrastructure as follows.

- We setup a DC/OS cluster with 1 bootstrap node, 3 masters nodes, 1 public node and 73 private nodes, which are going to be monitored. Remember that DC/OS is the container orchestrator that is going to deploy the FMonE pipelines. Further information about its parts can be found in their website ${ }^{26}$

- We divide the 73 private nodes in 4 regions to emulate a geo-distributed Fog environment:

- One region called "central region" which represents the upper cloud layer. This region has a Cassandra cluster installed, which will be used to measure performance with Yahoo Cloud Serving Benchmark (YCSB). In addition, this region also hosts a Kafka cluster, which we will later use to collect the monitored metrics, acting as a traditional centralised monitoring system. Both technologies will be hosted in 4 different nodes each so as to avoid any interference or overhead.

- In the remaining three regions, named "edge regions", we install the YCSB clients that are going to query the Cassandra database in the central region. This represents the communication between components at the edge and the cloud in terms of operations/sec. We will use this metric later to assess how a centralized monitoring approach can degrade the performance of the other components. The clients are configured to perform as many operations as they can per second until they complete 1000 operations.

- Between regions the communication bandwidth is restricted to $4 \mathrm{Mbps}$ and to emulate communication latency, we introduce a delay of $50 \mathrm{~ms}$ in all sent packets through the traffic control utility 27 . Bandwidth was chosen based on the statistics of speed testing in the European Union ${ }^{28}$ and taking into account that many of the Fog devices use technologies with variable speeds such as $4 \mathrm{G} / 3 \mathrm{G}$.

\footnotetext{
${ }^{26}$ https://docs.mesosphere.com/1.10/overview/concepts/

${ }^{27}$ https://linux.die.net/man/8/tc

${ }^{28}$ http://testmy.net/rank/countrycode.up/
} 


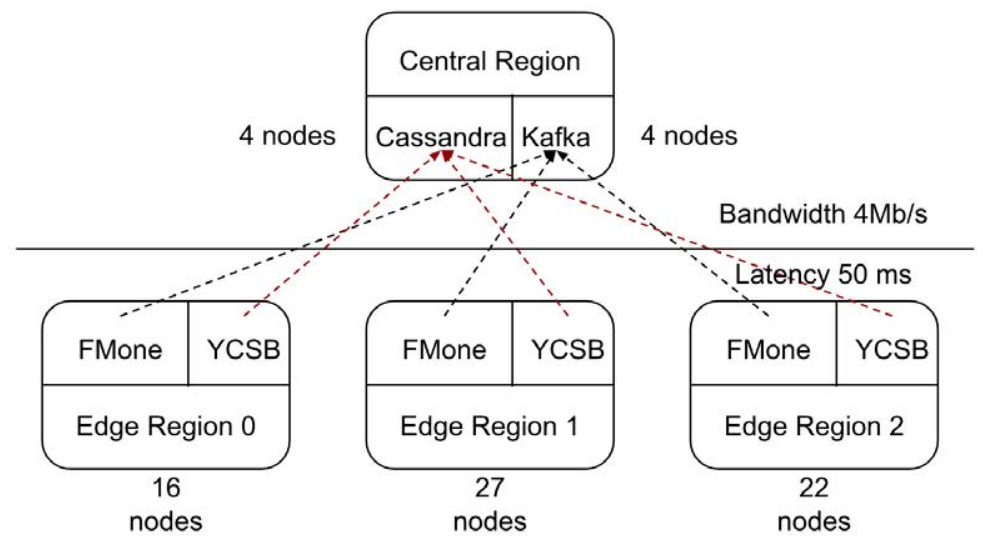

Figure 5.7: The configuration of our experiments. A central region hosts both a cluster of Cassandra and Kafka. On the edge, three regions of different size, host the YCSB clients communicating with the Cassandra database together with the FMonE agents sending their metrics to Kafka. Note that Kafka and Cassandra are running on different nodes to avoid overhead. Bandwidth and latency between regions are limited to $4 \mathrm{Mb} / \mathrm{s}$ and $50 \mathrm{~ms}$ respectively.

A diagram of the setup is depicted in Figure 5.7 for a better comprehension. Note that this is a simulated scenario and there are conditions not included, like routing the traffic through gateways. Hardware heterogeneity also comes to mind, but it is important to note that FMonE can allocate its agents in any device with a Docker daemon, which has been proved to work even in small single-board devices (134).

\subsubsection{Benefits on performance}

In our first experiment, we want to evaluate the impact on performance of an FMonE pipeline compared to a centralized approach. We configure the following scenario to do this:

- To simulate the applications and components that run inside Fog devices and that will be monitored by our agents, we launch 5 dummy Docker containers per VM that just sleep for a long period of time.

- Two FMonE agents are deployed in each node. One is going to collect 30 different types of metrics from the disk, memory, network and cpu usage at the host level. The second one will collect 24 different metrics about the resource usage of each of the five containers running in that node. 
- Metrics are sent every second, since we want to consider the settings of two possible scenarios that can occur in a Fog monitoring context. The first one is latency sensitive applications, like the previously explained online gaming use case, where alerts have to be raised almost in real time to act accordingly. The second is the post-mortem diagnosis, where users need to determine the exact source of what caused a component failure in their system. Both cases need a fine-grained level of detail and a continuous record of what is happening on the different components, which justifies the choice of one second as an interval.

- We measure the performance of the YCSB clients with the following FMonE pipelines:

1. Centralized: We send all the metrics to the Kafka queue in the central region. This could be considered as the baseline and the paradigm followed by most monitoring solutions, where everything is stored in a single back-end. Note that the nodes that host the Cassandra cluster used for the evaluation of YCSB and the ones that host the Kafka cluster are different. Otherwise we will create an obvious overhead in the database operations.

2. Aggregated: We have an FMonE agent in each region that is going to aggregate each type of metric for all the hosts of the same region, before sending them to the central location.

3. RegionalDB: All the agents of one region are going to store their metrics in a MongoDB back-end, which resides in that same region.

- In addition, to compare FMonE with existing monitoring solutions, we also measure the performance with a Prometheus (113) server in the central region, in the same vein as the centralized FMonE pipeline. Prometheus is an extensively used open-source monitoring toolkit that is based on a pull model, where metrics are extracted from monitoring agents that expose them through HTTP endpoints. Although the monitoring agents are not part of the Prometheus framework per se, there is a great variety of them developed by the open-source community. In our case, we launch a cAdvisor ${ }^{29}$ agent in each node, which exposes 52 metrics, a number close to the amount of metrics generated with the FMonE scenarios (30

\footnotetext{
${ }^{29}$ https://github.com/google/cadvisor
} 


\begin{tabular}{|c|c|c|}
\hline Workload & Description & $\begin{array}{c}\text { App } \\
\text { Example }\end{array}$ \\
\hline A & $\begin{array}{c}50 / 50 \\
\text { reads/writes }\end{array}$ & Session Store \\
\hline B & $\begin{array}{c}95 / 5 \\
\text { reads/writes }\end{array}$ & Photo Tagging \\
\hline $\mathrm{C}$ & $100 \%$ read & $\begin{array}{c}\text { Read User } \\
\text { Profile }\end{array}$ \\
\hline $\mathrm{D}$ & $\begin{array}{l}\text { Read latest } \\
\text { inserted }\end{array}$ & Status Update \\
\hline $\mathrm{E}$ & $\begin{array}{l}\text { Read short } \\
\text { range }\end{array}$ & $\begin{array}{c}\text { Threaded } \\
\text { Conversation }\end{array}$ \\
\hline $\mathrm{F}$ & $\begin{array}{c}\text { Read- } \\
\text { modify-write }\end{array}$ & $\begin{array}{c}\text { Record user } \\
\text { activity }\end{array}$ \\
\hline
\end{tabular}

Table 5.1: The different YCSB workloads

for host and 24 for containers). The system is configured to pull these metrics every second. We have chosen Prometheus because it is open source, easy to configure and it offers a high degree of customization for its agents. It is also widely adopted in current infrastructures and it is used in many cases together with popular container orchestrators like Kubernetes 30 .

We expect a drop in YCSB client performance for the centralized FMonE pipeline and the Prometheus scenario, since the limited bandwidth available to the device when communicating with the central region will have to be shared between the YCSB operations and the metrics sent by the monitors. We execute all of the workloads that are available by default. A table with the characteristics of the different workloads is depicted in Table 5.1. Note that since there are connection constraints between the YCSB clients and the central region where the Cassandra database resides, we expect a low number of operations per second. The clients execute each workload until they complete 1000 operations and this is repeated three times. The results can be seen in Figure 5.8. The operations per second throughput is averaged for the 65 nodes that

\footnotetext{
${ }^{30}$ https://techcrunch.com/2018/08/09/prometheus-monitoring-tool-joins-kubernetes-as-cncfs-latestgraduated-project/?guccounter $=1$
} 
host the YCBS clients and the three executions. Notice how workload E has a low number of ops/sec since read short range is an expensive operation. As expected, there is an improvement in the performance on all workloads with respect to the centralised Prometheus approach, reaching as much as $8 \%$ for some cases. It is important to note that these are the results for an emulated scenario in which we use the linux traffic control utility, while in a real scenario, this effect would be further exacerbated by the metrics generated by thousands of nodes and the additional stress on the backbone of the network (52). The aggregated and regionalDB approaches have similar performance as the traffic going out of each region is reduced to the minimum, thanks to the aggregation and flexible back-ends features of FMonE. In the former, only one aggregated metric is sent to the central region and in the latter, everything is stored within the monitored region. The flexible monitoring model that FMonE provides through the aggregation, filtering and geo-aware storage of the metrics, enables an optimal placement of the monitoring workflow, eliminating any impact on the performance of the applications.

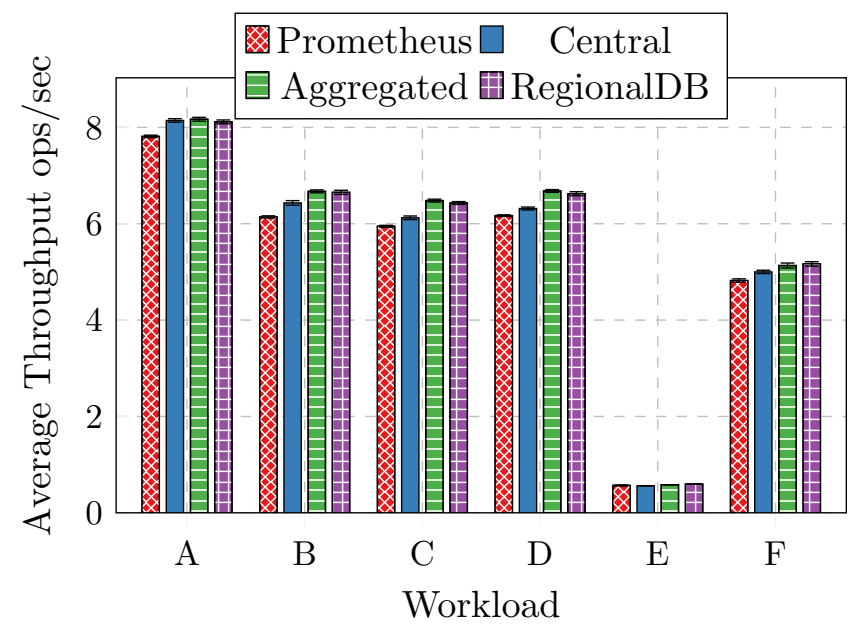

Figure 5.8: Impact on the performance of edge applications with different monitoring methods. A typical centralized approach, where all the metrics from the edge are sent to a central location, is compared to two FMonE pipelines that aggregate and store metrics in their own region, respectively. The traffic caused by the monitoring information consumes much of the limited bandwidth at the edge, affecting the number of ops/sec. 


\subsubsection{Overhead and resource usage}

The monitoring process should be lightweight and non-intrusive, specially in resourcepoor IoT devices. The aim of this experiment is to prove the requirement of nonintrusiveness of FMonE. We monitor the performance of the YCSB clients in each region in two situations: without any monitoring at all and with the previously introduced FMonE regionalDB pipeline, where the metrics from the host and from 15 additional containers per host are dumped to a MongoDB in their same region. Again, metrics are gathered and published every second and the YCSB clients perform requests until they complete 1000 operations. The results are depicted in Figure 5.9, averaged for the 65 nodes that host the YCBS clients. The overhead is minimal with a maximum drop of $1.2 \%$. The fact that the agent can keep the metrics in memory before publishing them, enables us to transfer the data in bulk, using resources in a more effective way. This, together with the fact that the FMonE agent is a lightweight container, means low impact on performance. We also show in Figure 5.10 the resource usage of the nodes hosting the different back-ends for the metrics. We mentioned that a fog environment has heterogeneous hardware. One of our requirements was deploying different backends depending on the computational power of the device that is going to host it. In this experiment we use different back-ends and show their different resource usage profiles. The objective is to motivate why back-ends should be chosen depending on the computational capabilities of the hosting device. We compare Prometheus with two of the different back-ends that are available in FMonE: MongoDB and Kafka. The latter was deployed as a cluster in 4 different hosts and the resource usage was averaged across them. This can be effectively used to divide the storage burden across different devices if needed. The Prometheus back-end has a higher CPU usage than other options as MongoDB or Kafka, indicating that Prometheus might not be suitable for a device with low CPU capacity. The percentage of memory used remains very similar for the three solutions with values of around 22 and $25 \%$. The disk usage graphic, reveals peaks of up to $25 \mathrm{MB}$ written to disk for Prometheus while Kafka and MongoDB show a more stable write pattern of a few MB per second. As it can be seen, different back-ends show different resource usage patterns. Detaching the back-end required by the monitoring solution and offering different options for the metrics storage enable a better planning 


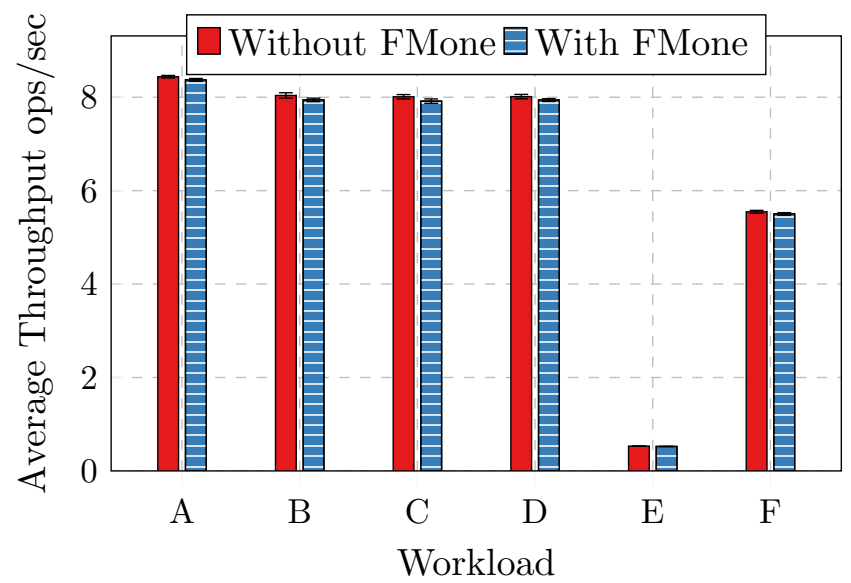

Figure 5.9: Overhead of a FMonE deployment. Even when monitoring 15 containers per host, the overhead is negligible for an FMonE pipeline.

of the monitoring workflow. Light back-ends can be used for fog devices with limited resources and more powerful and complex ones for upper layers near the cloud.

\subsubsection{Elasticity}

Fog devices can frequently join and leave the infrastructure, as stated in our requirements section. For instance, a mobile node that joins the network or a device that is switched on, has to be included again in the monitoring process. We need to assess how soon agents can be up and running on a device. We measure the time in seconds that it takes to deploy FMonE agents in the following configurations: 1 in one node, 2 in one node, 10 in 5 nodes, and 30 in 15 nodes. When using a Docker container, the node needs to pull down the image from a repository if it has not been done yet. We perform the test in both situations, where the node pulls and does not pull the image. The results are shown in Figure 5.11 with the average deployment time and standard deviation for 5 rounds of experiments. As expected, a node that runs FMonE for the first time introduces some overhead when downloading the image. This overhead will be higher the more agents we deploy at the same time, due to the time needed to download the image. The maximum is 34 seconds for 30 agents and 15 hosts. Bear in mind that this situation, where the node has to pull the Docker image, only happens once and it takes only 2.5 seconds to deploy them once the nodes already have the 


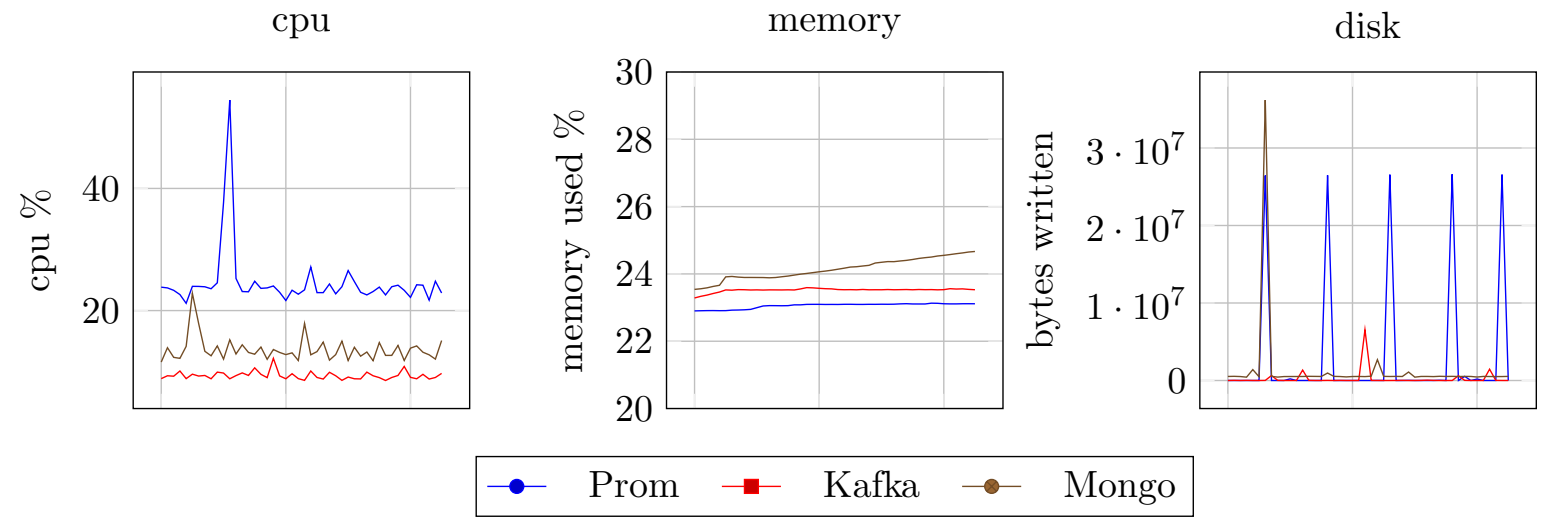

Figure 5.10: Time series of the resource usage for Prometheus and two of the available FMonE back-ends: Kafka and MongoDB. Prometheus is a more resource intensive backend compared to the other two. Note that the resource usage is important when placing the back-end for metrics on devices with limited resources such as Fog.

Docker image.

\subsubsection{Resilience}

Lastly, we test the resilience of all the FMonE parts. The following three scenarios are laid out where we introduce a series of failures that can happen in an unstable scenario such as Fog:

- We shutdown the FMonE agents running on a host and measure the time that passes between the shutdown and the agent recovery.

- We then shutdown the MongoDB instance. Since the agents do not have a backend to publish to, they will also exit with an error code. We measure the time interval for the whole pipeline to go up again.

- Finally we shutdown all the containers of the pipeline and the node containing the Marathon engine. Marathon is the foundation in which the FMonE operation is based on. However, it is also resilient by means of a Zookeeper instance (69), and is able to relaunch itself again by choosing a new leader. The pipeline is automatically restored afterwards. Again, this time interval is measured.

We repeat the experiment 10 times for each scenario. The mean time and its standard deviation are shown in Table 5.2. We can see how an agent can recover quickly 


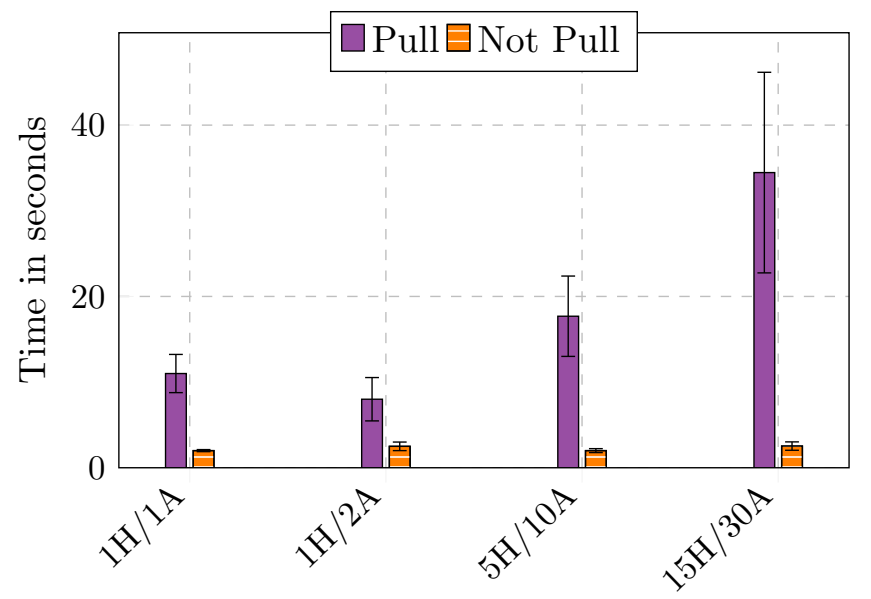

Figure 5.11: Time that it takes to start up the monitoring agents. We measure it when the container image needs to and does not need to be pulled from the Docker repository. We also vary the number of host and containers from 1 host and 1 agent to 15 hosts and 30 agents. Pulling creates an obvious traffic overhead compared to the fast deployment of a container that has already been pulled.

$\begin{array}{lll} & \text { Mean recover time } & \text { Standard deviation } \\ \text { Agent } & 6.637 \mathrm{~s} & 2.454 \mathrm{~s} \\ \text { Backend } & 27.602 \mathrm{~s} & 17.889 \mathrm{~s} \\ \text { Marathon + Pipeline } & 96.844 \mathrm{~s} & 34.627 \mathrm{~s}\end{array}$

Table 5.2: Resilience of the system

and lose only about 7 seconds of monitoring information. There is more overhead for a failure in a back-end, since these containers have more complex deployment times than the lightweight FMonE agent. Additionally, a failure in a back-end also affects all the other agents, as previously explained. Nonetheless, the maximum delay we observed was 58 seconds with a mean of 27 seconds for the 10 experiments. The most costly situation to recover is when Marathon fails. In this case the system has to elect a new Marathon instance and relaunch the FMonE pipeline. The system recovered in 96 seconds on average. Remember that this is an extreme situation, since the Marathon instance does not have to be running in any of the Fog nodes which are unstable and constrained devices. The idea is to have all the core components like the Mesos master, Marathon and the FMonE framework in a stable location from where the whole Fog 
infrastructure can be coordinated.

\begin{tabular}{|c|c|c|c|c|c|c|c|c|c|c|}
\hline & 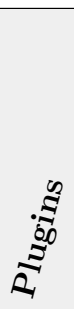 & 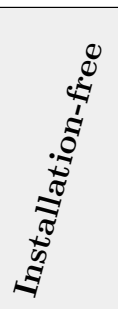 & 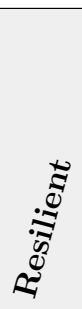 & 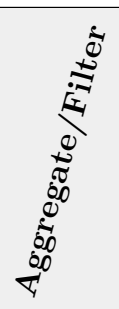 & 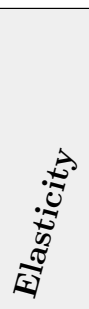 & 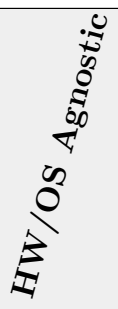 & 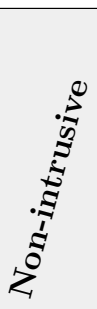 & 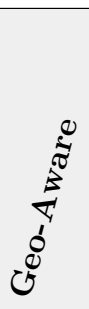 & 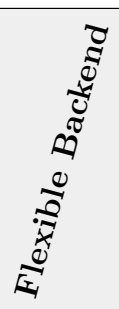 & 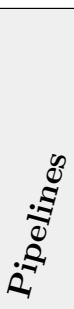 \\
\hline \multicolumn{11}{|c|}{ Academia } \\
\hline GMonE & $\checkmark$ & 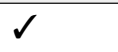 & & $\checkmark$ & & & $\checkmark$ & & $\checkmark$ & \\
\hline DARGOS & $\checkmark$ & & & $\checkmark$ & & & $\checkmark$ & $\checkmark$ & & \\
\hline PyMon & & $\checkmark$ & $\sqrt{2}$ & & 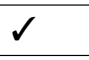 & $\checkmark$ & $\sqrt{2}$ & $\checkmark$ & & \\
\hline FAST & & & & $\checkmark$ & $\checkmark$ & $\checkmark$ & $\checkmark$ & & & \\
\hline Wu et al. & & & & & 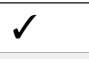 & 4 & & & & \\
\hline \multicolumn{11}{|c|}{ Industry } \\
\hline Nagios & $\checkmark$ & & & & & & & & & \\
\hline New Relic & $\checkmark$ & & & & & & $\checkmark$ & & & \\
\hline Prometheus & $\checkmark$ & $\checkmark$ & $\checkmark$ & & $\checkmark$ & $\checkmark$ & $\checkmark$ & & & \\
\hline CA APM & $\checkmark$ & $\checkmark$ & $\checkmark$ & & $\checkmark$ & $\checkmark$ & $\checkmark$ & & & \\
\hline Datadog & $\checkmark$ & 4 & $\checkmark$ & $\checkmark$ & $\checkmark$ & $\sqrt{2}$ & $\sqrt{2}$ & $\checkmark$ & & \\
\hline Dynatrace & $\sqrt{2}$ & 4 & $\sqrt{2}$ & 4 & 2 & $\sqrt{2}$ & 4 & 2 & & \\
\hline & & & & & & & & & & \\
\hline FMone & $\checkmark$ & $\checkmark$ & $\checkmark$ & $\checkmark$ & $\checkmark$ & $\checkmark$ & $\checkmark$ & $\checkmark$ & $\checkmark$ & $\checkmark$ \\
\hline
\end{tabular}

Table 5.3: Cloud and Fog monitoring properties.

\subsubsection{Qualitative evaluation}

As a summary, Table 5.3 includes the different features provided by some of the state-ofthe-art monitoring solutions that we described in Chapter 3.2 and how they compare to FMonE. These features overlap with the requirements for a Fog monitoring tool listed in Section 5.2. As it can be seen, industry products seem to provide more advance features than academic solutions. Monitoring has always been a big concern in Industry, and current monitoring solutions provide many of these functionalities. However, most of them are designed for a cloud scenario, where monitoring is centralized in one place. FMonE provides flexible back-ends and user-tailored monitoring pipelines across the system. This is important in a Fog environment, where we have a hierarchical structure with diverse hardware, allowing us to aggregate monitored data from the edge up to 
the cloud with different back-ends to choose from.

Although some monitoring solutions can be adapted to target a Fog architecture, none of them meet the whole set of requirements, falling short in one aspect or another. This has already been pointed out by recent studies in the Fog/Edge monitoring field and with FMonE we intend to fill that gap.

\subsection{Conclusions: Fog observability is possible and con- tainers are a key technology in IoT}

Fog computing is powered by a complex and heterogeneous infrastructure that is constantly evolving. This presents several challenges when ensuring the performance and quality of service of the applications they host. In this chapter, we have listed those challenges and presented the design of FMonE, a monitoring framework that flexibly coordinates highly customisable monitoring agents and their back-ends in order to provide detailed observability into the Fog system. We have demonstrated that this tool can satisfy the different requirements through a series of experiments that show its resilience, elasticity and non-intrusiveness. We have also showed that the centralised monitoring approach used by the cloud is not the best fit for Fog computing, as the many elements to be monitored will generate and overwhelming amount of metrics. Additionally, the devices that comprise the Fog have features such as location, connectivity or hardware resources that vary across the infrastructure and, therefore, the monitoring process should adapt accordingly.

Our experiments also show that container technologies and microservice architectures can enable the Fog computing paradigm. Same as with FMonE, other Fog applications can be deployed on disparate devices, such as smart phones or any other system as long as they support the deployment of containers. This also opens up the possibility of a lightweight container orchestrator for Fog that coordinates thousands of devices with limited resources. We have used DCOS to ease the execution of the experiments but a more lightweight container orchestrator like Hypriot Cluster Lab (53) might be needed when using resource-constrained devices at the edge.

In any case, FMonE implements the monitoring step needed to gather the intelligence needed by an autonomic system for the Fog. It is also a clear example of why microservice architectures are a good fit for the Fog computing paradigm. However, 
throughout the implementation of FMonE we also explored the complexity of managing and maintaining several containers running on different machines. Container orchestrators provide some support for this task, but they fall short in some other features like establishing the root cause of a failure. We believe this is a key feature in a DSS for infrastructure management and in the next chapter we describe a graph-based model that can be incorporated to solve these challenges. 


\section{Chapter 6}

\section{Root cause analysis for microservice architectures}

Containerisation is a virtualisation technique that is gaining wide adoption nowadays. Many businesses have already acknowledged their benefits and microservice architectures are being quickly adopted by some of the top companies in Industry (하). One of the systems that could benefit the most from the use of this type of virtualisation is the Fog infrastructure. When we introduced FMonE in the previous chapter, we already listed some of the advantages of using containers for Fog computing and its applications: elasticity, hardware and operating system agnostic, or resilience are some of the characteristics of containers that adapt to the peculiarities of the Fog.

Coordinating and scaling these containers require an orchestrating unit, specially if we want to deploy them in a distributed infrastructure with several machines. Tools like the previously mentioned Kubernetes or DC/OS have filled this gap. These platforms have self-healing features, where unhealthy or faulty containers can be relaunched, or containers migrated to a different machine in case one of their hosts dies. But establishing the root cause of these failures is an important step to apply the right contingency plan or to find ways to prevent them in the future. Nonetheless, root cause analysis (RCA) can become very complex, specially when we have a network of different, unique microservices that depend on each other. The troubleshooting process normally involves tedious search through logs across the different containers, trying to find the faulty piece in the chain.

In this chapter, we present a graph-based method to perform root cause analysis 
(RCA) in such a microservice architecture. We argue that a graph representation of the system is able to capture important information for RCA, like the topology of the architecture or the different connections, both logical and physical, between elements in the system. Based on this observation, we build a framework with two goals: allowing the user to better understand what is the current behaviour of the system (anomalous situations, metrics, communication between elements, etc...) and matching an anomalous situation in the system with a previously diagnosed situation. The objective is to build the intelligence needed by the DSS to troubleshoot and assist the user with any anomalies that occur in the architecture. The content of this chapter is based on the article "Graph-based Root Cause Analysis for Microservice Architectures", which is currently under review (21) and the article "Next Stop NoOps: Enabling CrossSystem Diagnostics Through Graph-based Composition of Logs and Metrics", which has been published in IEEE Cluster 2018 (152).

\subsection{Graph Based RCA}

To motivate why graphs are a good fit to represent microservices architectures, we use a common web serving scenario. In this architecture, we have a number of clients that perform HTTP requests to a load balancer, such as HAproxy 31 , which redirects these requests to a set of stateless web servers, for example WordPress ${ }^{32}$. The web servers also store any state information on a backend database, such as a traditional MySQL 33 server. From a microservice point of view, each of these elements is a container running on a machine of the cluster. Every container or host has its own metrics and communicates with other containers or hosts in the system. For example, the load balancer will communicate with all the web server instances in the architecture, but there will not be interaction between the web servers. Graphs allow us to formalize the logical and physical connections between the different elements of the system through edges. In addition, all of the metrics information can be included as attributes on the nodes, as seen in Figure 6.1. These attributes can be numerical values, such as metrics, categories, such as a label specifying if the node represents a container or a physical machine or even ontological classes that could help to model the domain, like knowing

\footnotetext{
${ }^{31}$ http://www.haproxy.org/

${ }^{32}$ https://wordpress.com/

${ }^{33}$ https://www.mysql.com/
} 


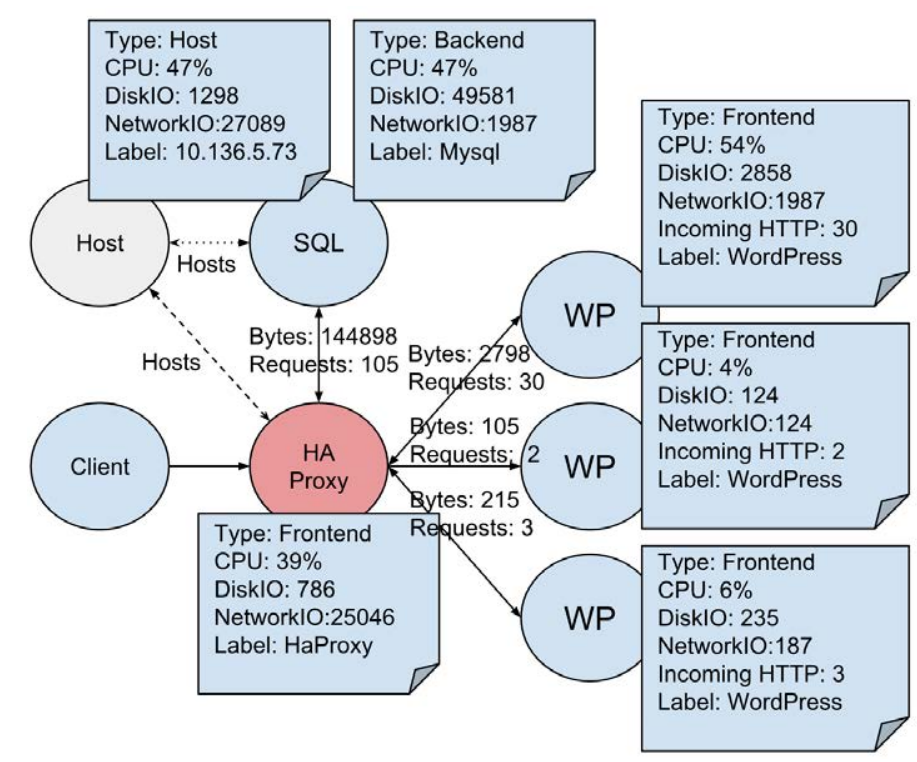

Figure 6.1: Simplified example of a multi-attributed graph model for a microservice architecture. An anomaly is generated in the HA Proxy instance due to the unbalanced number of requests that the WordPress instances are receiving

that WordPress and HAProxy are both frontends. Some other attributes, like vectors or information from logs, can be easily added in order to improve the system.

It seems logical, and experience tell us so (58), that any anomaly or drop in performance can have a cascading effect. In the case of the load balancer, it might propagate to the webservers as they will see less incoming requests than usual. Similarly, in a container environment, any anomaly in the functioning of the host might be propagated to the containers it hosts. The graph representation allows to identify this propagation effect, since we are able to determine which nodes or elements are connected to an anomalous one.

Based on this graph representation, which we will call system graph from now on, we propose a RCA system that is able to match an anomalous region in the system, represented as a subgraph, with a similar anomalous graph from the past that has already been troubleshooted by an expert. There are several pieces involved in this process represented as a general architecture in Figure 6.2. In the following subsections we will describe the architecture in more detail. Note that this approach can be applied to other systems such as service-based or to local processes that are not containerised. However, and as previously mentioned, the approach excels with microservice architec- 


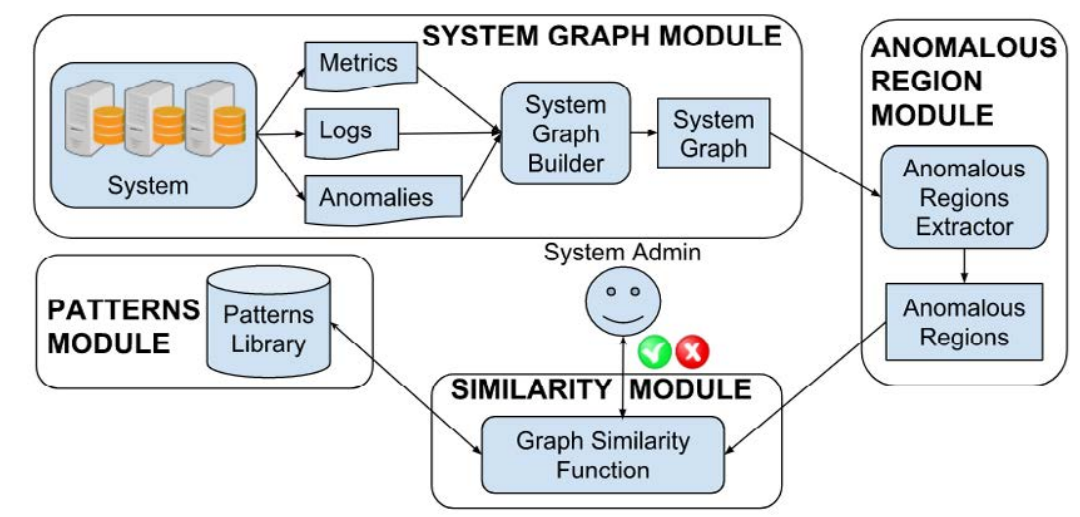

Figure 6.2: Architecture of the proposed system

tures since the different parts of an application are decomposed into units that interact between each other.

\subsubsection{The system graph module}

The responsibility of this module is to embed into a graph representation all the collected metrics, network activity, logs and anomalies generated in the system for a given time window (e.g. 15 secs). We can think about this graph as a snapshot of the current state of the microservice. The size of the window can be adjusted by the user based on criteria such as the architecture change rate or the monitoring frequency of the agents. The trade-off between a large and a too narrow time window needs to be considered. Larger time windows will result in some anomalies not being detected due to the coarse-grained information of the metrics. Too narrow ones will result in noisy information and a lot of overhead when collecting metrics, logs and anomalies.

The process involved in the graph creation for our particular system graph will be further explained in Section 6.3. Here we provide a general overview of the graph elements, which can be applied to any other context besides containers.

Nodes: Nodes represent either containers, hosts or any other element outside the cluster that interact with our system e.g. an external client accessing a frontend. Nodes may have multiple attributes of different types. We explain the different types further in this section.

Edges: Edges represent any network communication between elements in the system, such as TCP connections or HTTP requests. They also represent a logical con- 


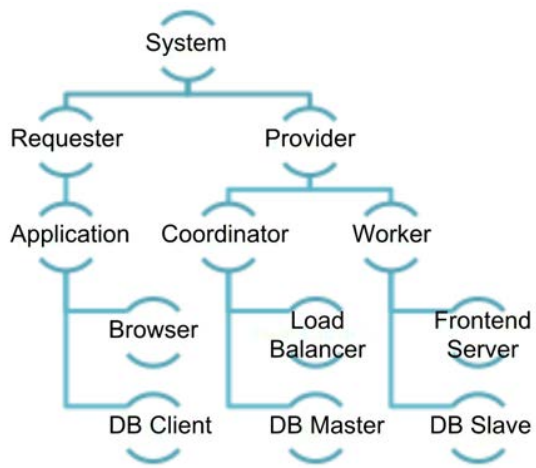

Figure 6.3: An example of a taxonomy explaining a hierarchy of concepts. When comparing graphs, the taxonomy will allow us to reason those concepts situated close to each other in the tree, concluding that they are more similar. For example a DB master is very similar to a load balancer, since they both are coordinators that distribute some load to their slaves.

nection, such as the relation between a container and the machine hosting it. Same as with nodes, edges may have multiple attributes of different types.

Attributes: Attributes are used to capture the information collected from metrics, logs and anomalies. We provide a list of the types considered in our framework:

- Numerical: Here we include metrics that can take any value over a discrete or continuous range. One obvious example will be the cpu usage of a container, but it can also be found in the edges. For instance, the number of bytes sent from one container to another.

- Categorical: They take values that are names or labels. One example would be the Docker image for a particular container (e.g. Haproxy v1.8, WordPress v4.9, etc.)

- Ontological: This attribute type corresponds to an ontology class and it is used by the system to represent a hierarchy of concepts. A simple example of an ontology in the form of a taxonomy is depicted in Figure 6.3. These kinds of attributes are useful to generalise problems that are semantically the same but involve different elements. For example in the loadbalancer scenario, it would have been equivalent to have Nginx instances instead of WordPress instances, since they are both Frontend Servers. 
Anomaly level: This can be considered as a special type of attribute, indicating that a given node or edge is experiencing an anomaly. As we will see later, any of the state-of-the-art anomaly detection mechanism can be used to identify problems and tag the relevant elements of the graph with this attribute (7). It will be used afterwards by the anomalous region extractor to build an anomalous subgraph out of the system graph.

\subsubsection{The anomalous region module}

The output graph of the previously described system graph module will be analyzed by the anomalous region module to extract the different anomalous elements of the graph. The anomalous region module consists of two submodules: (i) an anomalous detection system that is able to detect and tag anomalous nodes inside the system graph and (ii) an anomalous region extractor.

For the former, any of the state-of-the-art techniques for anomaly detection can be used (28). Note that we assume the existence of this submodule, as we focus on the problem of finding a root cause for the detected anomaly and not the detection of the anomaly per se.

The anomalous regions extractor is a function that generates an anomalous subgraph for each of the nodes that have been tagged as anomalous. Again, this is customisable since we can insert here any kind of function that is able to extract anomalous subgraphs from nodes or edges that have an anomaly level other than zero. An example is depicted in Figure 6.4, where a function creates an anomalous region by taking the neighbours up to a distance of 2-hops for each anomalous node.

As this function extracts a subgraph from the general system graph, it will also keep its nodes, edges and attributes.

Nodes, edges and attributes in the anomalous region can additionally have weights assigned, representing the importance of a given element in that anomalous region. For instance, an anomaly involving a malicious process consuming a lot of cpu in one machine, could have its cpu usage attribute weight increased for that node with respect to other attributes of the graph. These weights will be taken into account by the graph similarity module. We will see later how the user can change these weights, effectively including the user in the machine learning process. 


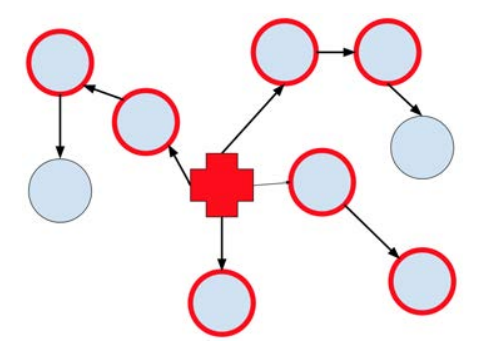

Figure 6.4: An illustration of the method that builds the anomalous regions. The anomalous node with the cross shape, generates an anomalous region including neighbours that are located at 1 or 2 hops from anomalous nodes, representing a cascading effect where the performance of one element depends on the others

The extracted anomalous regions are sent to the similarity module, in order to match them with already troubleshooted anomalies stored in the library.

\subsubsection{The pattern library module}

The pattern library is composed of a set of subgraphs representing the anomalous regions that have been already revised and labeled by an expert. These patterns will act as reference templates with which anomalous situations currently happening in the system can be matched. As we will see later in Section 6.1.4, patterns are added to the library if a new anomalous region is not similar enough (up to a given threshold) to any of the graphs currently present in the library. In addition to this, it can initially be filled manually or as a result of importing the pattern library from a different deployment. The objective is to have an initial set of the most common errors that can affect the system, in order to avoid the cold-start issue. The more patterns, the more complete this library will be, although it will also increase the search time. The user can access the library and visualize the different anomalous graph patterns, browsing their metrics, logs and connections. This visualisation will help the user to enrich the existing patterns by adding metadata through the following actions:

Changing weights: By having a graph representation of the system, the user can change the aforementioned weights and give more importance to certain nodes, edges or attributes. This will help in the RCA process since the important elements are pinpointed. Consequently, matching the pattern with any new incoming anomaly of the same type will be easier. 


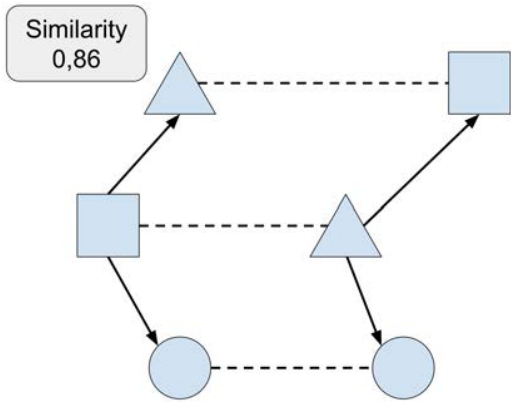

Figure 6.5: A representation of the mapping between nodes and the similarity score given by the graph similarity function

Cause label: It is a label that represents the main reason or root cause for the anomalous pattern. Same as with weights, it is normally assigned by an expert in the domain that analyses the graph structure and establishes a root cause. It can also come with a series of steps needed to fix the anomalous situation by the system administrator or with automatic prescriptive actions that can be triggered if the pattern occurs.

\subsubsection{The similarity module}

The similarity module relies on an inexact matching function that considers the similarity distance between two multi-attributed-weighted graphs. The module receives two inputs: an anomalous region generated by the anomalous region module and a potentially tagged pattern from the pattern library module. The output consists of two elements: a similarity score and a mapping of nodes from graph A to graph B. The former goes from 0 to 1, 1 meaning that the two graphs are identical and 0 that they are completely different. The latter describes which graph elements from the first graph are matched with the elements from the second graph to achieve this similarity score. A visual example of this function is depicted in Figure 6.5. Note that the details of the function can be implemented in different ways, applying any state-of-the-art graph matching techniques. We give the details of our function in Section 6.2.

Every time a new anomalous region arrives, the module tries to match it with one or more graph patterns of the library. We consider that there is a match between two graphs, when the similarity value between them is above a user-specified threshold. If there is no match, the anomalous region is added as a pattern, waiting for an expert to troubleshoot and tag it. If there is one or more, the list of matched patterns can be 
handled in different ways. We can show it to the user as a ranked list with their root causes (labels). Then the user can validate or reject the different matchings. While the validation means that the matching is aligned with the ground truth, rejection indicates that the comparison did not capture the underlying information in the graphs. Another option is ordering the list by similarity and applying an automatic healing procedure based on the top-similar root cause. We leave this criteria open as it depends on the domain and the criticality of the situation.

The threshold element creates a trade-off between correctly classified anomalies and search time inside the library. Decreasing the threshold will result in more patterns being inserted in the library, more options to compare with and higher accuracy in the classification, as we will see later in the evaluation section. However, more patterns will obviously mean longer search times. Some heuristics that can be used are setting a relatively high threshold and verify if the classification error is acceptable or the threshold should be decreased. We empirically found that a threshold between 0.80 and 0.90 is able to define well the difference between two different anomalous situations. Finally, we would like to point out that correctly tuning the weights will allow the user to set higher thresholds since the similarity scores will be higher between graphs sharing a common root cause and having their most important elements pinpointed.

\subsection{Graph similarity engine}

One of the central pieces of the framework described in the previous section is a function able to find a similarity score between two graphs. In this section, we give the details of our graph similarity function implementation and the particularities that allow introducing expert knowledge from system administrators.

\subsubsection{Problem definition}

Definition 1. A graph $G$ is represented by a 5-tuple:

$$
G=(V, E, a t t, C, w)
$$

$V$ is a set of vertices. $E \subseteq V \times V$ is a set of edges. att is a function such that $\operatorname{att}(V \vee$ $E)=A$, where $A$ is a list of attributes $a$ represented by tuples $a=\{$ val, weight, $c \subset C\}$, with $v a l$ being its value, weight being the weight or importance of the attribute inside 
that node or edge and $c$ being the context of the attribute. $C$ represents the graph context and is used to explain the attributes that are included in the graph and to give a context in which to compare its values, as we will see later on the section. $w$ will be a function such that $w(x)$ returns either the weight of an edge or node within its graph, or the weight of an attribute within its node or edge.

Definition 2. Given two graphs:

$$
G_{1}=\left(V_{1}, E_{1}, a t t, C, w\right), G_{2}=\left(V_{2}, E_{2}, a t t, C, w\right)
$$

And two injective functions $m_{n}: V_{1} \rightarrow V_{2}$ and $m_{e}: E_{1} \rightarrow E_{2}$, the former returning the matched node of $G_{1}$ into $G_{2}$ and the latter returning the same for edges, we have an optimisation problem where we want to find the mapping that maximises the similarity between the two graphs, given by the formula:

$\frac{\sum_{v \in V_{1}}\left(w(v)+w\left(m_{n}(v)\right)\right) \cdot \operatorname{sim}\left(v, m_{n}(v)\right)+\sum_{e \in E_{1}}\left(w(e)+w\left(m_{e}(e)\right)\right) \cdot \operatorname{sim}\left(e, m_{e}(e)\right)}{\sum_{v \in V_{1}} w(v)+\sum_{v \in V_{2}} w(v)+\sum_{e \in E_{1}} w(e)+\sum_{e \in E_{2}} w(e)}$

$$
\underset{m_{n}, m_{e}}{\arg \max }
$$

where $\operatorname{sim}(x 1, x 2)$ is a function that represents how similar two nodes or two edges are with a score from 0 to 1.

The above formula formalizes that the similarity score is the weighted average of the similarities between the optimally mapped elements of the graph through the functions $m_{n}$ and $m_{e}$. We also have to emphasize the importance of the weights of the nodes $\left(w(v)+w\left(m_{n}(v)\right)\right)$ and edges $\left(w(e)+w\left(m_{e}(e)\right)\right)$ in the calculation. Elements that have a bigger weight or importance in the graph will contribute more to the global similarity score. This can be used to pinpoint the nodes that are more important within the context of the problem.

The minimization problem can be solved with any of the state-of-the-art techniques available like $\mathrm{A}^{*}(118)$. In our case we use hill climbing, which in comparison to $\mathrm{A}^{*}$, is a continuous optimization problem, reducing execution time at the expense of finding a local maximum instead of a global one.

At this point, we have to explain two elements present in the Equation $6.2 \operatorname{sim}\left(v, m_{n}(v)\right)$ and $\operatorname{sim}\left(e, m_{e}(e)\right)$. In other words, the similarity between one node and its mapped 
node, and the similarity between one edge and its mapped edge. We detail the implementation of these comparisons in the following subsection. For quick reference, we also include the notation used in Table 6.1.

\subsubsection{Similarity between nodes and edges}

The solution for the optimal mapping found in Equation 6.2 depends on the similarity between nodes and edges. The similarity between two graph elements (edges or nodes) $x_{1}$ and $x_{2}$ is given by the following equation:

$$
\frac{\sum_{a \in \operatorname{att}(v) \cup \operatorname{att}(u)}\left(w\left(a_{1}\right)+w\left(a_{2}\right)\right) \cdot \operatorname{sim}\left(a_{1}, a_{2}\right)}{\sum_{a \in \operatorname{att}(v) \cup \operatorname{uatt}(u)}\left(w\left(a_{1}\right)+w\left(a_{2}\right)\right)}
$$

Where $\operatorname{sim}\left(a_{1}, a_{2}\right)$ is the similarity between the attributes of the elements, which we will explain in the next Subsection 6.2.3. Similarly to Equation 6.2, this formula expresses the idea that the similarity between two elements is a weighted average between all of their shared attributes.

Analogously to Equation 6.2, the importance of the weights $w\left(a_{1}\right)+w\left(a_{2}\right)$ given to each attribute is used to modify the comparison calculation. Increasing the weight of an attribute means that the similarity of two nodes or two edges will increase even more if they have similar values for that attribute. An example of this effect can be seen in Figure 6.6, where giving a bigger weight to an attribute, like the shape of the nodes, prioritizes shape similarity over structure similarity, since the matching node on the right neither has two edges nor is connected to a circle. This an useful feature that enables including domain knowledge into the graph. An anomalous region where it is clear that the problem is the disk, may have the weights of all the disk-related attributes increased. When searching for patterns in the library, similar values for this metric will be prioritized.

\subsubsection{Similarity between attributes}

In this subsection, we explain in detail how we calculate $\operatorname{sim}\left(a_{1}, a_{2}\right)$, in other words, how we compare the multi-type attributes of the elements in the graph. Here it is where the contexts that we defined in the graph structure (Equation 6.1) come into play.

When comparing elements, the context helps us to measure the similarity between their different attributes, like ontological concepts (e.g. comparing a backend to a 


\begin{tabular}{|c|c|}
\hline Notation & Description \\
\hline$G$ & $\begin{array}{l}\text { A graph represented by a tu- } \\
\text { ple }\{V, E, a t t, C, w\}\end{array}$ \\
\hline$V$ & The vertex of a graph \\
\hline$E$ & The edges of a graph \\
\hline att & $\begin{array}{l}\text { A function that returns a list } \\
\text { of attributes a for a given } v \in \\
V \text { or } e \in \mathrm{E}\end{array}$ \\
\hline$a$ & $\begin{array}{l}\text { An attribute of a given } v \\
\text { or } e \text {, represented by a tuple } \\
\{\text { val, weight }, c \in C\}\end{array}$ \\
\hline val & The value of an attribute \\
\hline weight & $\begin{array}{l}\text { The weight of an attribute in- } \\
\text { side the vertex or edge it be- } \\
\text { longs to }\end{array}$ \\
\hline$c$ & $\begin{array}{l}\text { The context of an attribute. } \\
\text { It is used to calculate the sim- } \\
\text { ilarity between two attributes } \\
\text { provided they have the same } \\
\text { context }\end{array}$ \\
\hline$C$ & $\begin{array}{l}\text { The context of the graph. It is } \\
\text { used to explain the attributes } \\
\text { that can be found in the graph } \\
\text { and their different contexts } c\end{array}$ \\
\hline$w$ & $\begin{array}{l}\text { A function that returns the } \\
\text { weight of any given } v \text { or } e \\
\text { within its graph, or the weight } \\
\text { of an attribute inside its } v \text { or } \\
e\end{array}$ \\
\hline$m_{n}$ & $\begin{array}{l}\text { An injective function that } \\
\text { matches the vertex of a graph } \\
G_{1} \text { to a vertex of a graph } G_{2}\end{array}$ \\
\hline$m_{e}$ & $\begin{array}{l}\text { An injective function that } \\
\text { matches the edge of a graph } \\
G_{1} \text { to an edge of a graph } G_{2}\end{array}$ \\
\hline $\operatorname{sim}(x 1, x 2)$ & $\begin{array}{l}\text { Function that calculates the } \\
\text { similarity score between two } \\
\text { vertex, edges or attributes }\end{array}$ \\
\hline
\end{tabular}

Table 6.1: Notation table 


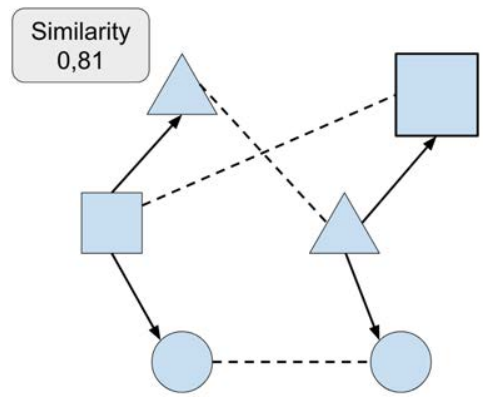

Figure 6.6: Effect of weights in the matching algorithm. Giving a bigger weight to the shape attribute of the nodes changes the matching in comparison to Figure 6.5. In this case the structure of the graph is not as important as matching nodes with the same shape.

frontend) or metrics that are bounded (e.g. cpu usage going from 0 to 100). The contexts are assigned by the user for each of the metrics or attributes that are used to build the graphs depending on their nature. The representation of this context $\mathrm{C}$ depends on its type and it will be used in the different similarity calculations. If an attribute does not have a valid context, it will be ignored in the similarity calculation. We consider three different types of contexts in our graphs:

Categorical Context: An attribute $a_{1}$ with a categorical context will have a label as its value (e.g. kafka representing the Docker image used by that container). When comparing two attributes with a categorical context, and since they normally represent labels or names, we use an exact equality function:

$$
\operatorname{sim}\left(a_{1}, a_{2}\right)= \begin{cases}1 & \text { if } a_{1}=a_{2} \\ 0 & \text { otherwise }\end{cases}
$$

Numerical Context: A numerical context is represented as a tuple $c_{\text {numerical }}=$ $\{\min , \max \}$ where min and max represent the minimum and maximum value that an attribute with that context can take (e.g. \% of cpu usage, which can range from 0 to 100). When comparing two attributes with a numerical context we use the function:

$$
\operatorname{sim}\left(a_{1}, a_{2}\right)=1-\frac{\left|a_{1}-a_{2}\right|}{\max -\min }
$$

Ontological Context: An ontological context is represented as a tree data structure, where each node represents a concept in the microservice architecture. The goal of this type of context is to enable the comparison between two elements that are similar in nature (e.g. a loadbalancer and a distributed database master perform similar tasks: 
redirecting requests to a pool of backends). When comparing two attributes with an ontological context, we use a variation of the Wu and Palmer similarity metric (146): let $c 1$ and $c_{2}$ be two concepts in the ontology, and $C$ be its closest common ancestor, then $\operatorname{sim}(C 1, C 2)=\frac{2 \cdot d(C)}{d(C 1)+d(C 2)}$, where $d(x)$ is the number of nodes to traverse from the root of the taxonomy to concept $x$ (we include always $x$ in that number, thus $d(x) \geq 1)$.

The advantage of such a modular approach is that we can design new contexts for our attributes. This is also where the usefulness of contexts in the graph comparison engine resides. So far we included these three ones, but we could also create a new context for metrics with different comparison criteria. This is specially useful in the root cause analysis domain. For instance, a stressed system with a value of cpu of $100 \%$ compared to a value of $80 \%$, can mean a lot more than just a $20 \%$ difference. We can also develop distribution-aware contexts, where attributes with values that fall outside a given distribution (e.g. outliers) will have more weight in the comparison result. We actually develop this idea further in Section 6.5.

\subsection{Monitoring and building the graphs}

In Section 6.1 we described the architecture of the RCA framework and its different parts. So far we have explained our own implementation of the anomalous region module in Section 6.1.2, the design of the pattern library module in Section 6.1.3 and the graph similarity function in Section 6.2. In this section, we explain the implementation details to build the system graph representation of the microservice architecture through the system graph module. Our objective is to illustrate the extraction of the different attributes of the nodes and edges and how we define the connections between the different elements of the graph. For this task, we have used Sysdig 24 and Prometheus 35 but other monitoring tools can be used, as long as there is a plugin that transforms the input of metrics into a graph. In the following subsections we explain the technical details on how we build these graphs.

\footnotetext{
${ }^{34}$ https://www.sysdig.org/

${ }^{35}$ https://prometheus.io/
} 


\subsubsection{Adding the nodes}

We start off by adding the nodes that will form part of the graph, i.e the elements that are alive in the system (containers and hosts). The size of the graphs depends mainly on the number of components involved in the microservice architecture as we will see later in the evaluation section.

We need to extract metrics at two different levels here: container level and host level. To extract these metrics, we run two containers in each machine: node exporter 36 and cadvisor $[37$. The former will take care of monitoring the host and the latter the containers running on the different hosts.

As a back-end for the metrics collected we choose Prometheus, which is going to pull the information coming from cadvisor and node exporter instances running on each node. The metrics are stored following a multi-dimensional time series data model. This means that for each metric we will have a series of tuples like ( $t s, i d$, value, labels), where $t s$ is a milisecond-precision timestamp, $i d$ represents which container or host the metric belongs to, value is the metric value (e.g. 100\% for cpu usage) and labels are a set of optional key-value pairs representing particular dimensions for that metric.

As an illustration, for the node_cpu metric we could have a data point like ( $t s=$ $1518537794351, i d=10.136 .1 .9$, value $=98.1, c p u=c p u 3$, mode $=$ user $)$, representing that the metric node_cpu at timestamp 1518537794351, for the node 10.136.1.9 had a value of 98.1 in the core cpu3 in user mode. This allows us to slice the data by different dimensions. Since our goal is to build a snapshot of the system for a given time window, we just have to use the time dimension $t s$ to extract the metrics over one period. In our case, we want to extract all the available metrics for the container and hosts alive at every second. We chose this time window since the monitor agents that we use publish their metrics every second and it also gives us enough granularity to classify the different anomalies. Each distinct $i d$ is going to be added as a node in the graph and its information contained in the pairs of (value, labels) inserted as attributes for that $i d$.

\footnotetext{
${ }^{36}$ https://github.com/prometheus/node_exporter

${ }^{37}$ https://github.com/google/cadvisor
} 


\subsubsection{Adding the edges}

Once we have the nodes, we can start connecting them through their edges. To achieve that, we need to monitor the network communication inside the microservice architecture. This is specially challenging since containers, unless told otherwise, use the IP of the host and its TCP port space, hindering the process of capturing traffic per container. Luckily, container orchestration tools facilitate the creation of overlay networks. These networks create a separate network namespace where each container will have its own IP and hostname. We use this feature together with Sysdig in each machine to capture any TCP communication between elements. Sysdig is an open source tool that instruments the Linux kernel and captures every system call and OS event. This trace of events can also be dumped into trace files in a very similar way to tools like tcpdump 38 Sysdig is also able to differentiate these events per container out-of-the-box.

Ultimately, we will obtain some of the same dimensions from sysdig as those used to slice the Prometheus data in the previous subsection. In fact, and summarizing, for each TCP request we will have a tuple ( $t s, i d$, cip, sip, io_dir, bytes), where $t s$ is the timestamp for that request, $i d$ is the container or host in which that communication happened, cip is the client IP, sip is the server IP, io_dir is whether it is reading or writing into the TCP socket and bytes is the amount of information sent or read in bytes. Remember that sip and cip are unique among containers thanks to the overlay network. Analogously to the Prometheus data processing, we use the $t s$ value to extract all the TCP communications between containers for a given time window. Then we group all of the entries within that window by (cip, sip) and count the number of entries in each group and the sum of its bytes, representing the number of TCPrequests and the size of the information sent respectively. As a result, each group is going to have the information sent through the TCP pipe cip $\rightarrow$ sip in the direction io_dir for two different $i d 1$ and $i d 2$ elements involved in that pipe. Remember that the $i d$ for the elements were added as nodes in the previous step. Therefore, we just need to add an attributed edge from $i d 1$ to $i d 2$ and vice versa, where the attributes are going to be the previously mentioned count of TCPrequests and the sum of bytes in each io_dir. We depict an example of this process in Figure 6.7.

\footnotetext{
${ }^{38}$ https://www.tcpdump.org/tcpdump_man.html
} 


TCP pipe cip -> sip
\begin{tabular}{|llll|}
\hline id & io_dir & count & sum \\
\hline id1 & write & 4 & 128 \\
\hline id1 & read & 10 & 1024 \\
\hline$i d 2$ & write & 10 & 1024 \\
\hline$i d 2$ & read & 4 & 128 \\
\hline
\end{tabular}

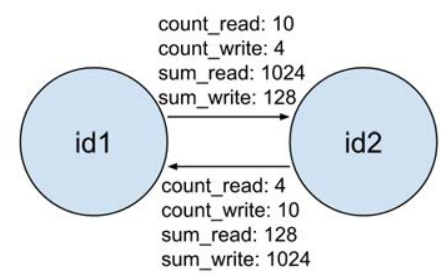

Figure 6.7: An example on how we group the different TCP requests traced by sysdig. Each group is going to have four entries for each client ip $\rightarrow$ server ip TCP pipe that will be added as attributes of the edges

\subsection{Evaluation of the graph-based approach}

In this section, we evaluate the accuracy of the RCA framework and the effect of its different features, like its weights and thresholds. To do that, we create an experimental environment where we introduce anomalies in three different microservices scenarios, common in industrial environments. We first explain the scenarios (Section 6.4.1), followed by the anomalies triggered in them (Section 6.4.2). Afterwards, we describe the set-up used to run these microservices architectures in a real environment such as the Grid'5000 testbed (14) (Section 6.4.3). We conclude with an evaluation of the different features of the RCA method.

\subsubsection{Microservices scenarios used}

To test our RCA graph approach, we have chosen a set of microservice architectures with several connected components. All of these architectures have been implemented as Marathon application group\$39. We list here the different architectures designed:

Loadbalancer scenario: This is a common scenario used in production, where a system receives a high number of HTTP requests that need to be balanced (27). A loadbalancer (HAProxy) acts as a frontend, receiving HTTP requests from a series of clients represented as Apache Benchmark Docker images 40 . HAProxy balances these requests to a set of 16 WordPress instances that will return a simple blog website. WordPress needs a MySQL database, which is also going to sit behind the loadbalancer. A graphical representation of this setup is shown in Figure 6.8 .

\footnotetext{
${ }^{39}$ https://mesosphere.github.io/marathon/docs/application-groups.html

${ }^{40}$ https://httpd.apache.org/docs/2.4/programs/ab.html
} 


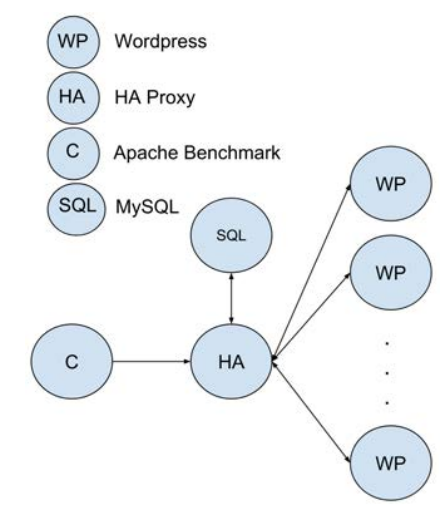

Figure 6.8: A simple representation of the loadbalancer scenario

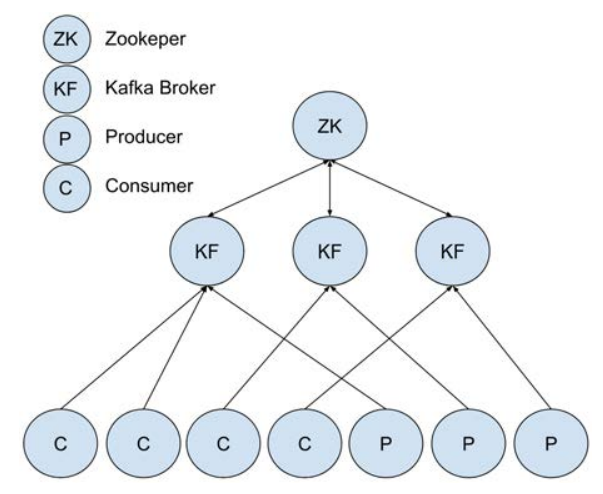

Figure 6.9: A simple representation of the Kafka scenario

Kafka cluster scenario: Kafka is a distributed messaging system used to collect or send high amounts of data. It is commonly used in many Big Data stacks (42) and its architecture consists of a set of brokers that store the messages, producers sending those messages and consumers that pull the data from the brokers. There are no master nodes as the brokers are coordinated by means of a Zookeeper instance. We implement this architecture with 1 Zookeper instance, 4 Kafka broker containers, 12 producers, which are monitoring agents sending to the brokers system metrics of the host they are running in, and 12 consumers that are pulling those metrics from the Kafka brokers. A simplified depiction of these architecture is shown in Figure 6.9.

Spark and HDFS scenario: Same as with Kafka, the combination of Spark and HDFS (126) is a widely deployed architecture in Big Data. Both of them have a master-slave architecture, with the master coordinating a series of worker nodes to process and storage data in parallel. In this case, we will have a highly interconnected 


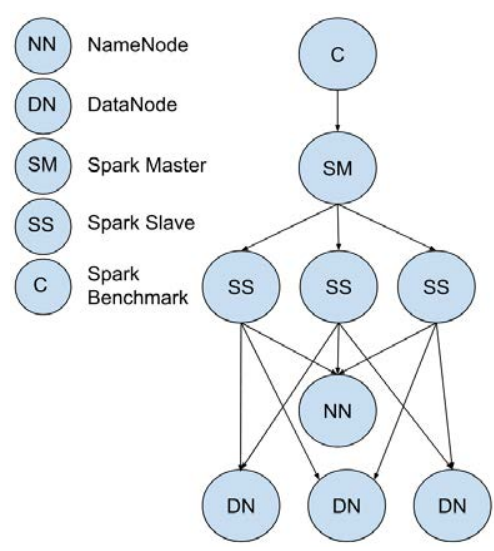

Figure 6.10: A simple representation of the HDFS + Spark scenario

graph structure with the Spark workers writing/reading data from the different HDFS DataNodes and reading metadata from the NameNode. Figure 6.10 represents this mesh of communicating containers. In our case, we will have 4 DataNodes, 1 NameNode, 4 Spark slaves and 1 Spark master. Additionally, we will use a container that has the SparkBench benchmark, as a means to send data analytics jobs to the Spark master. In the experiments, we use a workload inside this benchmark that generates a dataset in a comma-separated format, writes it to disk and then reads it again to transform it to the Apache Parquet data format 41

\subsubsection{Anomalies triggered in the scenarios}

In order to test the RCA technique, we need to generate different anomalies and evaluate if our system is able to distinguish their root causes. To accomplish this, we use several tools that allow us to stress and change the normal behaviour of the system:

Stressing hosts: stress-ng 42 is a tool that can stress several aspects and resources of a machine. Even if this anomaly is only applied directly in the hosts, containers running on those hosts will be affected if they use the same stressed resources. In this case, we will use four different types of stress tests:

- cpu: starts 6 workers that are going to exercise the cpu

- disk: starts 6 workers that will constantly write, read and remove temporary files

\footnotetext{
${ }^{41}$ https://parquet.apache.org/

${ }^{42}$ http://kernel.ubuntu.com/ cking/stress-ng/
} 
- network: starts 6 workers that are going to perform several connects, sends and receives on the localhost, simulating a socket stress

- bigheap: starts 1 worker that is going to grow its heap by constantly reallocating memory.

Connection problems: A drop in the bandwidth available for the machines or delays in communications are two possible problems in a distributed infrastructure (110). This has a clear impact on performance, specially in data intensive applications, where there is a large amount of exchanged data. We also emulate these kinds of conditions through the traffic control utility 43 (tc), a linux tool that allows manipulating the behaviour of the kernel when sending data through the network. Same as with stress-ng, this anomaly is only induced in the host, affecting the containers running on top. In our case, outgoing bandwidth is reduced to $75 \mathrm{kbps}$ and a delay of $100 \mathrm{~ms}$ is introduced to all packets leaving that node.

Wrong loadbalancing: There are several causes that can force a loadbalancer to unevenly spread the workload (114). We emulate this condition in the loadbalancer scenario, by login into the container and altering its HAProxy configuration files. By doing so, we overload all of the WordPress instances running on a given host, by redirecting 10 times more requests than the others.

Stressing endpoint: Endpoints can be flooded with requests, overloading the system and affecting the user experience. This can happen because of malicious attacks or just by peak demands (88). We stress the endpoint of our loadbalancer scenario, by running a set of Apache Benchmark Docker $\sqrt{44}$ images with several client threads that perform a large number of HTTP requests.

\subsubsection{Experiments set-up}

As previously explained, we implemented our microservices scenarios in DC/OS. In order to deploy them, we configure a cluster in the Grid'5000 testbed. We provision 8 VM's in the Rennes site with 16GB of RAM and 4 vcores. These machines will form a DC/OS cluster with 1 bootstrap node, 1 master node, 1 public node and 5 private nodes. Further information about DC/OS architecture can be found in their websit 45 .

\footnotetext{
${ }^{43}$ https://linux.die.net/man/8/tc

${ }^{44}$ https://httpd.apache.org/docs/2.4/programs/ab.html (Last accessed March 2018)

${ }^{45}$ https://docs.mesosphere.com/1.10/overview/concepts/
} 
To build a sequence of system graphs, representing the evolution of the cluster during our experiments, we do as follows:

1. We monitor six executions of each of the explained scenarios. During these executions, we inject the following anomalies:

- The stressing hosts and connection problems anomalies for 8 seconds in two random machines of the cluster.

- The wrong load balancing and stressing endpoint anomalies for 8 seconds in the HAProxy container for the loadbalancer scenario. For the former, we increase the load for all the WordPress instances that are running on a randomly selected host.

2. With the monitored metrics we build a set of system graphs for each second.

3. We tag the anomalous nodes corresponding to the anomalies we injected. This gives us a set of system graphs with their anomalous nodes.

This process corresponds to the system graph module explained in Figure 6.2, in which all the metrics and anomalies are gathered from the system in order to build graphs. The maximum size of the graphs built for these microservice architectures are 45 nodes for the Spark scenario, 63 for Kafka and 59 for the loadbalancer. We need to take into account, that the anomaly extractor region's module will limit the anomalous region from all these nodes and edges to a smaller graph. This region will be the graph that will be compared with the ones in the library.

Remember that we assume the existence of an alerting system that is able to raise an anomaly based on the metrics gathered. This could be easily achieved with any anomaly detection technique since stress-ng and $t c$ have a significant impact on the metrics at system level. In the wrong loadbalancing and stressing endpoint cases, the metrics can be extracted via the Unix socket of HAProxy, which includes the number of requests balanced to each backend 46 . We monitor six different executions to have six folds of data and use four of them as a training set and the remaining two as an evaluation set. This setup is further explained in the next subsection. The anomalies are injected into two random nodes to create some variability in the anomalous graphs generated, since each machine is going to host different containers.

\footnotetext{
${ }^{46}$ https://www.datadoghq.com/blog/how-to-collect-haproxy-metrics/
} 


\subsubsection{Precision of the system}

The objective of this experiment is to evaluate the accuracy of our system when matching the different anomalous regions of the evaluation set into the patterns found in the training set. Remember that we have six folds of data corresponding to six executions in our experimental environment. We perform the evaluation as follows:

1. For the first four executions, we take their system graphs, and group them by scenario and type of anomaly (e.g. spark cpu stress, kafka disk stress, load balancer stressing endpoint, etc...).

2. We process each of these groups with the RCA framework. In the process, the anomalous regions will be extracted. Remember that the anomalies are induced for 8 seconds in two random nodes for each scenario. That means 16 anomalous regions for each scenario/anomaly group, except for the wrong load balancing and stressing endpoint anomalies, with 8 anomalous regions, as they are induced in the single HAProxy container. We start with an empty library. The matching threshold is set to 0.9. This means that any anomalous region that is not similar up to $90 \%$ with a pattern, will be added to the library as a new pattern. This will generate a set of patterns representative of that scenario/anomaly group. Once the whole group is processed, we save the library, empty it and process the next group. The idea is to merge afterwards all of these saved libraries into a big library of patterns that will represent the training set, where each pattern is going to use the scenario/anomaly from where it originated as the classification label.

3. The graphs of the remaining two executions will constitute the evaluation set and are processed with the library populated during training. The threshold is lowered to 0 in order to avoid the addition of new patterns and forcing the system to always choose from the ones in the library. The RCA framework will match the different anomalous regions of these two executions with the patterns that were generated through points 1 and 2. From all the possible matched patterns we take the one with the highest similarity and assume the same root cause for the new situation. 


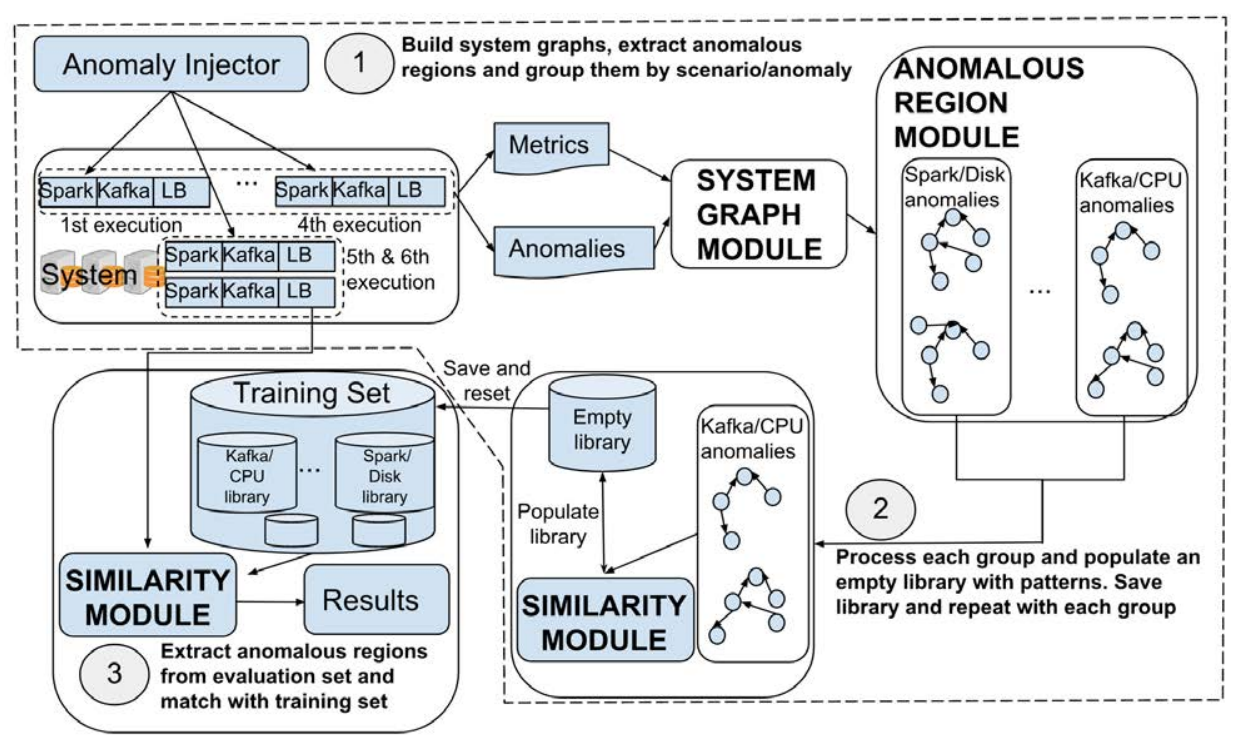

Figure 6.11: Design of the experiments to evaluate the RCA framework precision. There are two main steps. First, we process the first and second execution of each scenario to

build its system graphs, generate its anomalous region and then, for each anomaly, generate a representative library of patterns for that problem. All of these representative

libraries will be combined into one that will be used to establish the RCA of the anomalies induced in the third execution, based on the previously generated patterns.

We depict the whole process in Figure 6.11. Since we also want to test the previously explained weight system, we will run this experiment twice. In the first run, we do not tune the weights, while in the second run we tune the weights of certain attributes as an expert system admin would do.

Results are shown in Table 8.1 in a similar way to a confusion matrix, where each anomalous region is classified with its corresponding scenario/anomaly label (the root cause). For the sake of space, we just use the first letter of the anomaly on the left column. In each cell there are two values, which represent the percentage of correctly classified anomalous regions: the one on the left is the result without tuning weights and the one on the right by tuning them. The sum of the values in each column for both the weights and no weights cells will be $100 \%$. We consider that the distinction between the different scenarios (Kafka, Spark and loadbalancer) must be taken into account when performing root cause analysis, even when the cause of the anomaly is the same (cpu, disk, bandwidth or heap). We do so because the different elements involved in the anomaly could change the way to deal with the problem. For instance, 
even if our cpu stress anomaly is caused for the purpose of this experiment by stress-ng in all the scenarios, in a real set-up it could have different causes depending on the architecture and the containers running on that host (e.g. a Spark worker or a Kafka broker). Likewise, the prescriptive action can change, such as stopping non-critical containers, which also depends on the architecture. Therefore, this distinction between scenarios is needed.

\begin{tabular}{|c|c|c|c|c|c|c|c|c|c|c|c|c|c|c|c|}
\hline & \multicolumn{4}{|l|}{ KAFKA } & \multicolumn{4}{|l|}{ SPARK } & \multicolumn{6}{|l|}{ LB } \\
\hline & & CPU & DISK & BAND & HEAP & CPU & DISK & BAND & HEAP & $\mathrm{CPU}$ & DISK & BAND & HEAP & CONF & STRESS \\
\hline & C & $0.43 / 0.87$ & $0.12 /-$ & $0.03 /-$ & $0.18 /-$ & $-/ 0.125$ & - & - & - & - & - & - & - & - & - \\
\hline$\frac{1}{1}$ & $\mathrm{D}$ & \begin{tabular}{|l|}
$0.18 /-$ \\
\end{tabular} & $0.71 / 0.81$ & $\mid-/-$ & $0.15 /-$ & - & $-/ 0.03$ & - & - & - & - & - & - & - & - \\
\hline$\sum$ & B & $0.03 /-$ & $-/-$ & $0.90 / 1$ & $0.15 / 0.06$ & - & - & - & - & - & - & - & - & - & - \\
\hline & $\mathrm{H}$ & $0.34 /-$ & $0.12 /-$ & \begin{tabular}{|l|}
$0.06 /-$ \\
\end{tabular} & $0.5 / 0.71$ & - & - & - & $-/ 0.06$ & - & - & - & - & - & - \\
\hline & C & $-/ 0.12$ & - & - & - & $0.59 / 0.84$ & \begin{tabular}{|l|}
- \\
\end{tabular} & $0.21 /-$ & $0.15 /-$ & - & - & - & - & - & - \\
\hline 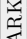 & $\mathrm{D}$ & - & $-/ 0.09$ & - & - & $0.12 /-$ & $0.25 / 0.87$ & - & $0.18 /-$ & - & - & - & - & - & - \\
\hline 虫 & B & - & - & - & - & - & $0.18 /-$ & $0.34 / 0.78$ & $0.06 /-$ & - & - & $-/-$ & - & - & - \\
\hline & \begin{tabular}{|l}
$\mathrm{H}$ \\
\end{tabular} & - & - & - & $-/ 0.03$ & $0.28 /-$ & $0.56 /-$ & $0.21 / 0.31$ & $0.59 / 0.59$ & - & - & - & - & - & - \\
\hline & C & - & - & - & - & $-/ 0.03$ & - & - & - & $0.28 / 1$ & $0.28 /-$ & - & $0.21 /-$ & - & - \\
\hline & $\mathrm{D}$ & - & $\begin{array}{l}0.03 / 0.09 \\
\end{array}$ & - & - & - & $-/ 0.09$ & - & - & $0.09 /-$ & $0.12 / 1$ & \begin{tabular}{|l}
- \\
\end{tabular} & $0.09 /-$ & - & - \\
\hline$\oplus$ & B & - & - & - & - & - & - & $0.18 / 0.124$ & - & $0.06 /-$ & $-/-$ & $0.96 / 0.96$ & $0.31 / 0.28$ & - & - \\
\hline & $\mathrm{H}$ & - & - & - & $-/ 0.18$ & - & - & $0.03 / 0.06$ & $-/ 0.343$ & $0.52 /-$ & $0.59 /-$ & $0.03 / 0.03$ & $0.37 / 0.71$ & - & - \\
\hline & C & - & - & - & - & - & - & - & - & - & - & - & - & $1 / 1$ & - \\
\hline & S & - & - & - & - & - & - & - & - & - & - & - & - & - & $1 / 1$ \\
\hline
\end{tabular}

Table 6.2: Confusion matrix for the graph based RCA method

There are several observations we can drawn from these results. The diagonal that crosses the table and that is highlighted in grey identifies the correct cells in which the classified anomalous regions should fall into. Any values not in the diagonal represent missclassified anomalous regions. For instance, without tuning weights $56 \%$ of Spark Disk anomalies are missclassified as Big Heap anomalies because in both of them there is a high number of bytes written to disk. We can observe that our RCA method is really effective with anomalies that have a significant effect on the elements around the anomalous node. For the BAND (connection problems), CONF (wrong load balancing) and STRESS (stressing endpoint) anomalies, the precision is near $100 \%$ without tuning any weights with the exception of the Spark scenario. In the BAND case, limiting the bandwidth of one machine has an effect on the performance of not only the containers running on that host, but also in all the containers in other machines that have to interact with them, creating a propagation effect that is easier to detect with a graph representation. For example, if one of the Kafka brokers has to send data to a consumer and its host has the upload bandwidth limited, the metrics for the whole application are going to be greatly affected. The STRESS and CONF anomalies show the same 


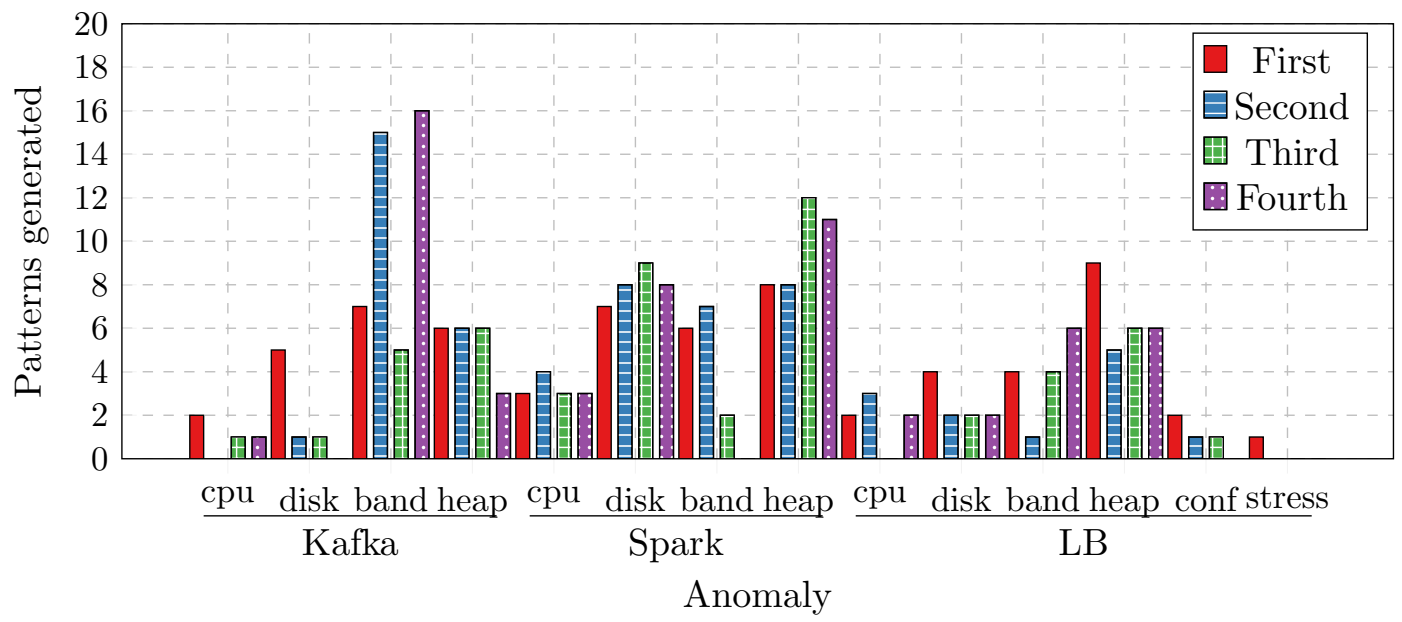

Figure 6.12: Number of patterns generated for the first, second, third and fourth execution of the training process

behaviour. Stressing an endpoint increases significantly the values in the HAProxy container for both the incoming/outgoing traffic attribute in the edges and the cpu usage of all the WordPress servers. Therefore the graph of the system is going to look very different to those of the other anomalies. A wrong load balancing problem means that some servers behind the frontend are going to receive a more significant part of the workload, resulting also in a distinctive graph pattern that the system is able to easily identify.

Another observation is that anomalies affecting a couple of host metrics, like DISK or CPU from the stressing hosts group, are difficult to match when weights are not properly tuned. We have to consider that from the whole anomalous region, only the attributes of one host in the graph change with respect to other anomalies. This is a very small part in the whole picture and makes it difficult to establish differences between a big heap problem (HEAP) and a CPU one affecting the same node, since they both intensively use the cpu. Here is where the expert knowledge comes into play in the form of weights. By increasing the weight of the anomalous host respect to other elements in the graph and pinpointing the important metrics of each anomaly in that host, the precision is $100 \%$ for some cases, like DISK and CPU for the load balancer scenario. In fact, from the 416 anomalous region generated, 349 are matched with the right pattern resulting in a precision of $83 \%$ overall when tuning the weights. 


\subsubsection{Number of patterns generated}

With this experiment we want to measure the amount of different patterns that each anomaly generates. In Figure 6.12, we show the number of patterns that were generated for the first, second, third and fourth execution of the training phase with the 0.9 matching threshold. For space reasons, we show the results only for the run in which we tuned the weights, although we add a discussion of the results without weights at the end of this section. The number of patterns generated stays low specially for the load balancer scenario and most of the cpu anomalies. This means that the system is able to capture most of the information in the first executions. The connection problems and big heap anomalies are the ones generating the most number of patterns. The reason behind this is that both anomalies cause intermittent loss of connection between containers, which translates into missing edges from one timestamp to the next. This will create a lot of structural dissimilarity between graphs, hindering the matching with any of the patterns of the library. In fact, this effect can be seen on the bandwidth anomaly for Kafka, in which for the second and fourth execution a large number of patterns were generated since there were no matchings. In addition, for the big heap case, a lot of variability can be seen in different metrics as the anomaly evolves, accentuating this situation even more. The total number of patterns generated are 237 out of 832 anomalous regions that were processed. If we compare this with the execution without weights, 84 patterns are generated for Kafka, 150 for Spark and 70 for the load balancer scenarios. This is a total of 304 patterns and a difference of 67 patterns between the two approaches meaning that tuning the weights not only increases the precision of the classification method, but also reduces the size of the library.

\subsubsection{The effect of thresholds}

In the previous experiments we considered that a match between an anomalous region and a pattern in the library happens if the similarity is greater than 0.9. It seems logical that decreasing that threshold will translate into a smaller library of patterns, since the matching requirement is relaxed. However, precision could decrease, since we will have less variety of patterns in the library to match with, while a smaller library will mean less patterns to label. To explore this trade-off between precision and library 
size, we try two different values for the matching threshold: 0.8 and 0.9 . In Figure 6.13 , we show the results for the precision as bars and the patterns generated as a line plot. In many cases, like in the CONF, DISK and some BAND anomalies, the higher threshold increases the precision, since we have more information captured in the library thanks to a wider set of patterns. On the other hand, having more patterns decreases the precision of BAND for the load balancer scenario. A finer tuning of the weights would be needed in this case, since some of these anomalous regions are matched with the numerous patterns for the Spark and Kafka scenarios. The maximum number of patterns generated is 43 for the connection problems in the Kafka scenario. This may seem like a excessive number of cases to troubleshoot by an administrator but we have to take into account that many of them are generated from graphs representing consecutive time windows. Therefore, the administrator could label the whole sequence of patterns with the same root cause without an extensive analysis.

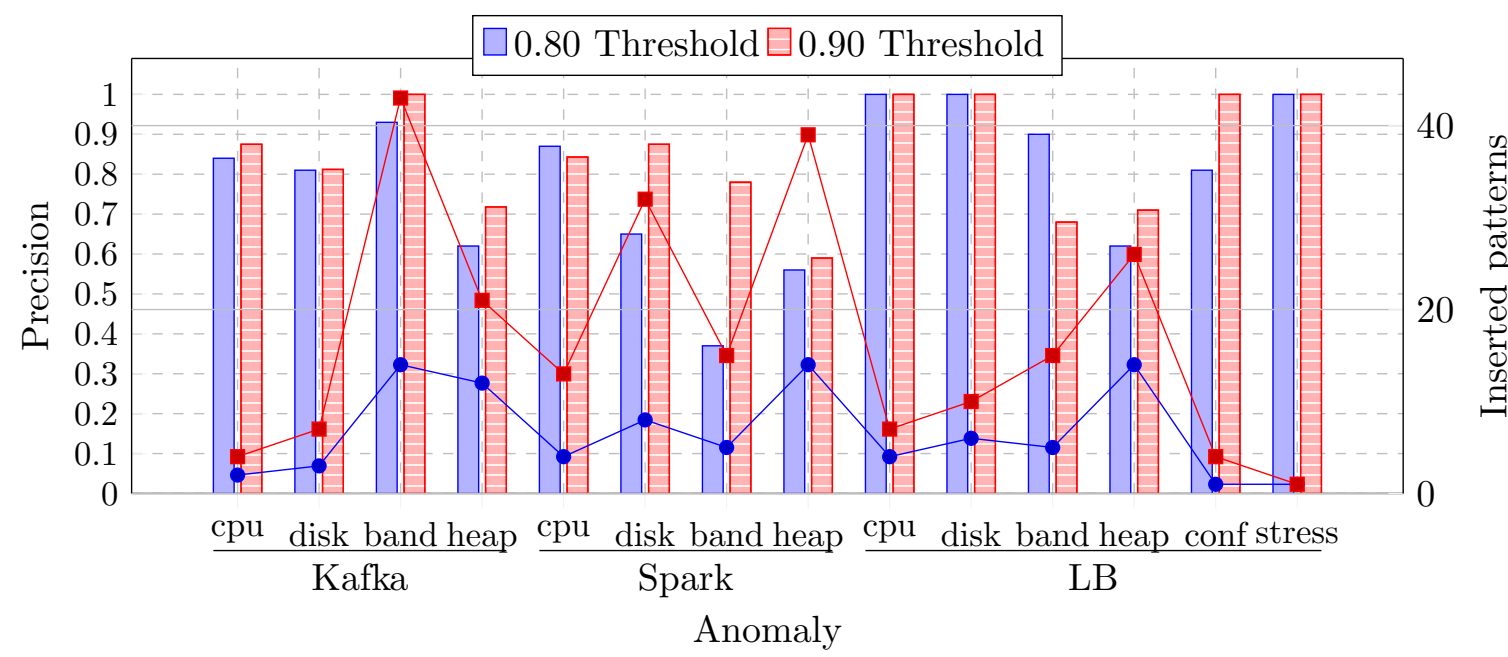

Figure 6.13: The effect of changing the matching threshold in the system. Precision is depicted as bars and the number of patterns generated as a line.

\subsubsection{Graph approach vs machine learning}

We also want to evaluate the benefits of the graph approach, where the topology of the system and the context around the anomaly are considered, versus a machine learning approach, in which a model is automatically learned from metrics of the anomalous 
element to predict its root cause (38). To achieve that, we train a boosted classification trees model with a dataset that contains the metrics for the anomalous nodes as features and the anomaly that was induced in that node as the classification label. Boosted classification trees were chosen because of their ability to work well with few data and their good accuracy (41). The comparison between the graph approach and the machine learning one is shown in Figure 6.14. We also detail its results in a confusion matrix in Table 6.3. The precision of the graph approach is higher in most of the cases. The expert knowledge embedded in the graphs through the weights and the extra information provided by the context surrounding the anomaly is specially useful in anomalies like CONF or BAND. Classification trees outperform its graph counterpart only in the cpu stress cases, since the problem is well differentiated by the peak experienced in the $c p u$ usage metric. We can conclude that the graph approach, enables a more precise and fine-grained root cause analysis, specially in such diverse and interconnected systems such as microservices.

\begin{tabular}{|c|c|c|c|c|c|c|c|c|c|c|c|c|c|c|c|}
\hline & \multicolumn{4}{|c|}{ KAFKA } & \multicolumn{4}{|c|}{ SPARK } & \multicolumn{6}{|l|}{ LB } \\
\hline & & $\mathrm{CPU}$ & DISK & BAND & HEAP & $\mathrm{CPU}$ & DISK & BAND & HEAP & $\mathrm{CPU}$ & DISK & BAND & HEAP & $\mathrm{CONF}$ & STRESS \\
\hline \multirow{4}{*}{ 倿 } & $\mathrm{C}$ & 0.93 & - & - & - & - & - & - & 0.18 & 0.125 & - & - & - & - & - \\
\hline & $\mathrm{D}$ & - & 0.68 & - & - & - & - & - & - & - & - & - & - & - & - \\
\hline & $\mathrm{B}$ & - & - & 1 & 0.06 & - & - & 0.40 & - & - & - & 0.5 & 0.28 & - & - \\
\hline & $\mathrm{H}$ & - & - & - & 0.65 & - & - & - & - & - & - & - & - & - & - \\
\hline \multirow{6}{*}{ 崩 } & $\mathrm{C}$ & 0.06 & - & - & - & 0.93 & - & - & - & - & - & - & 0.06 & - & - \\
\hline & $\mathrm{D}$ & - & 0.25 & - & - & - & 0.68 & - & - & - & 0.18 & - & - & - & - \\
\hline & $\mathrm{B}$ & - & - & - & - & - & - & 0.37 & - & - & - & 0.06 & 0.03 & - & - \\
\hline & $\mathrm{H}$ & - & - & - & - & - & - & - & 0.43 & - & - & - & - & - & - \\
\hline & $\mathrm{C}$ & - & - & - & - & 0.06 & 0.31 & - & - & 0.875 & - & - & - & - & - \\
\hline & $\mathrm{D}$ & - & 0.06 & - & - & - & - & 0.06 & - & - & 0.81 & - & - & - & - \\
\hline \multirow{4}{*}{ 坣 } & $\mathrm{B}$ & - & - & - & - & - & - & 0.15 & - & - & - & 0.43 & - & - & - \\
\hline & $\mathrm{H}$ & - & - & - & 0.28 & - & - & - & 0.37 & - & - & - & 0.62 & - & - \\
\hline & $\mathrm{C}$ & - & - & - & - & - & - & - & - & - & - & - & - & 0.5 & 0.06 \\
\hline & $\mathrm{S}$ & - & - & - & - & - & - & - & - & - & - & - & - & 0.5 & 0.93 \\
\hline
\end{tabular}

Table 6.3: Confusion matrix for the boosted decision trees RCA method

\subsubsection{Graph comparison latency}

Latency is an important factor in providing a quick response to fix the anomaly and stabilize the system. In this section we show the time needed to compare an anomalous region to a pattern in the library. In our experiment we compare two randomly selected graphs with sizes ranging from 10, 15, 20, 25 and 30 nodes. The experiment is repeated 30 times for each size combination to calculate the mean and variance of the execution 


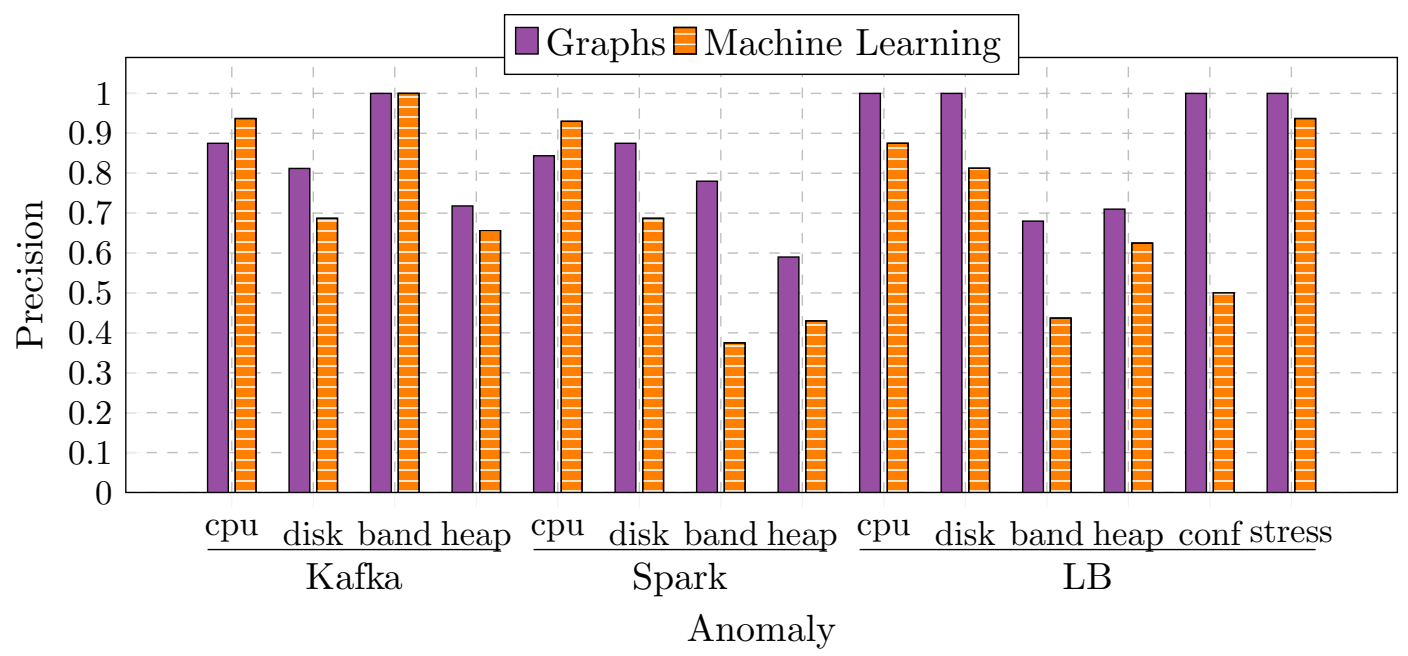

Figure 6.14: Precision of the graph-based approach compared to a machine learning based approach

time. The results are shown in Figure 6.15. Clearly, the comparison time depends on the number of elements that form part of the graph. The maximum execution time is 0.64 seconds for two graphs with 30 nodes each. Also the variance increases slightly as more nodes are involved in the process. Remember that the framework compares any incoming anomalous region with all the labeled patterns in the library, searching for the most similar one. This results in a sequential search, where a comparison needs to be done ntimes, where ntimes is the number of graphs in the library. As the library grows, establishing the root cause for that anomaly becomes more expensive. There are techniques that allow to index this search space (17) and we leave as future work their implementation to reduce the total search time in the library.

\subsection{Transferring knowledge through log analysis and met- rics distribution}

So far we have seen how graphs are an effective approach to model microservice architectures and the interactions between containers. Besides being a powerful visualisation tool, they also enable the use of artificial intelligence techniques to model specific parts of its behaviour. In our case, we modelled the root cause analysis of anomalies raised in a microservice architecture. One of the main advantages of this approach is that we 


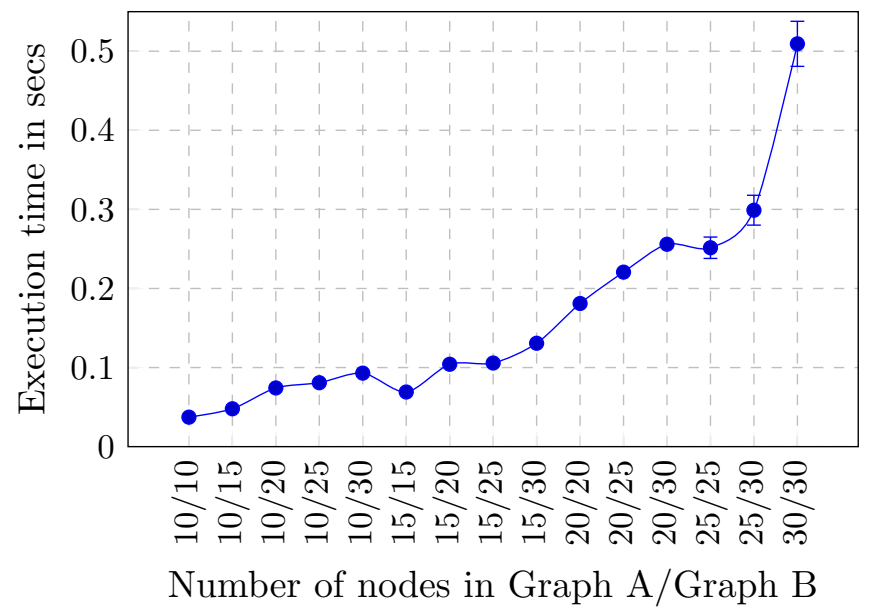

Figure 6.15: Execution time of the comparison between two graphs with different number of nodes.

do not need huge amounts of data to have an accurate model, as it is based on a library of patterns. As long as we have a labelled pattern, the system will be able to find a match, and subsequently, a root cause.

However, many AI techniques assume that the training data has the same feature space and same underlying distribution as the test data. This means that the model cannot be used with new data coming from a different domain, feature space or distribution of values. Even so, many of the correlations that were found by a given model in one source domain, can be leveraged and applied in a different target domain. This is commonly seen in human learning (109). For instance, a person that knows how to play one instrument, will find it easier to learn a different one. In the context of our work, we can consider the training data as the graphs in the library, and the test data as the incoming anomalous graph. Anomalies related to a master-slave architecture, can have the same reasoning process applied to the root cause analysis, independently of the software used (Spark, Flink, HDFS, etc.). Therefore we could use the graphs captured for one system to diagnose the other. This is called transfer learning (89). In this section we explain the work of Michal et al. (152) and how we contributed to fulfill this challenging task in microservices by leveraging three different techniques:

1. Inexact graph matching and similarity score between two graphs with different structures using the adjustable similarity functions (Subsection 6.2). The tech- 
niques that we already introduced, facilitate the comparison of graphs from two different domains. Also, defining a taxonomy allows for the calculation of the similarity between two nodes that are different but represent the same concept in a domain. For instance, a Spark slave server and a Hadoop DataNode will be near each other inside the taxonomy, as they are both slaves of a master-slave architecture.

2. Inclusion of logs in the graph representation, as they describe in natural language events that happen in the system, independently of their architecture or resource usage (Subsection 6.5.1). This abstracts completely the events from any metric or specific feature, enabling their comparison even if they are from different domains.

3. Including the information contained in the distribution of the metrics for a given architecture. We do it through automatic weight assignment (Subsection 6.5.2) as calculating weights will automatically pinpoint relevant metric values independently of the domain. We also add a similarity function based on the distance between distributions to our tool belt of existing functions (Subsection 6.5.3). This allows to compare metrics based on their distribution as it might change depending on the type of feature or metric used for comparison. Since different domains will use different types of metrics, this provides a much more consistent way of comparing them.

\subsubsection{Including log data}

Text provides a concise way of describing system events through natural language in a way that can be commonly understood by users. In this subsection, we propose a log representation structure that can be embedded into our graph as a vector attribute. In order to do that we need to introduce a new vector type attribute and a new context for their comparison.

\subsubsection{Vector attribute to represent logs}

Vectors can represent the status of one part of the system as an array of elements. In this case, the status will be given by the log messages generated during a given time window. Using techniques such as Word2Vec (100), we can create word embeddings for a given time window. Same as when we extracted metrics from the system, we need to 


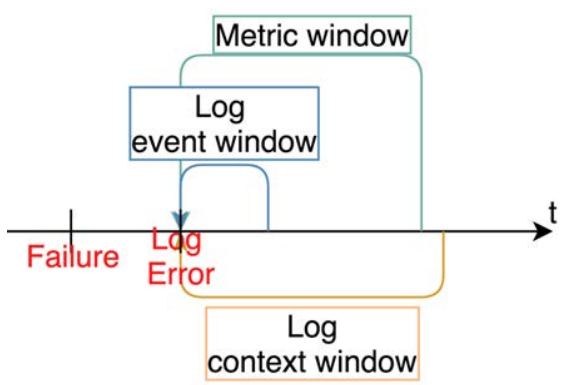

Figure 6.16: Scheme presenting context and event window of logs. Both windows start on a first Error or Warning message.

define which size we will use for the time windows. Using smaller ones will capture a more detailed meaning of that log slice. Larger ones will capture the context (general context of the application used). We use two log windows: a context window and a event meaning window. The context window gives a broader idea of the state of the system, capturing the normal activities of the application. The event meaning window captures log entries for a short time after an important event happens. Both windows start when an error or warning message is written into the log. The concept of window lengths is explained in Figure 6.16.

After we extract the text corresponding to the two time windows from the log, we perform the following steps:

- We remove special char sequences like hex strings, having as a result a much shorter vocabulary. Also, removing special characters helps to avoid model overfitting.

- We perform a tokenization step, where sentences are disassembled into bags of words.

- Using Word2Vec, we transform log into vectors. Firstly, we train the Word2Vec model, mapping the vocabulary into an $n$ dimensional space. A considerable advantage of using a Word2Vec embedding model is that it performs well even if it is trained using the vocabulary of one domain and used for another one. Also, similarity calculations should be as fast as possible to enable diagnostics of failures in a dynamic environment. Hence, it is not feasible to use NLP techniques such as key terms extraction. After that, each of the words for that particular 


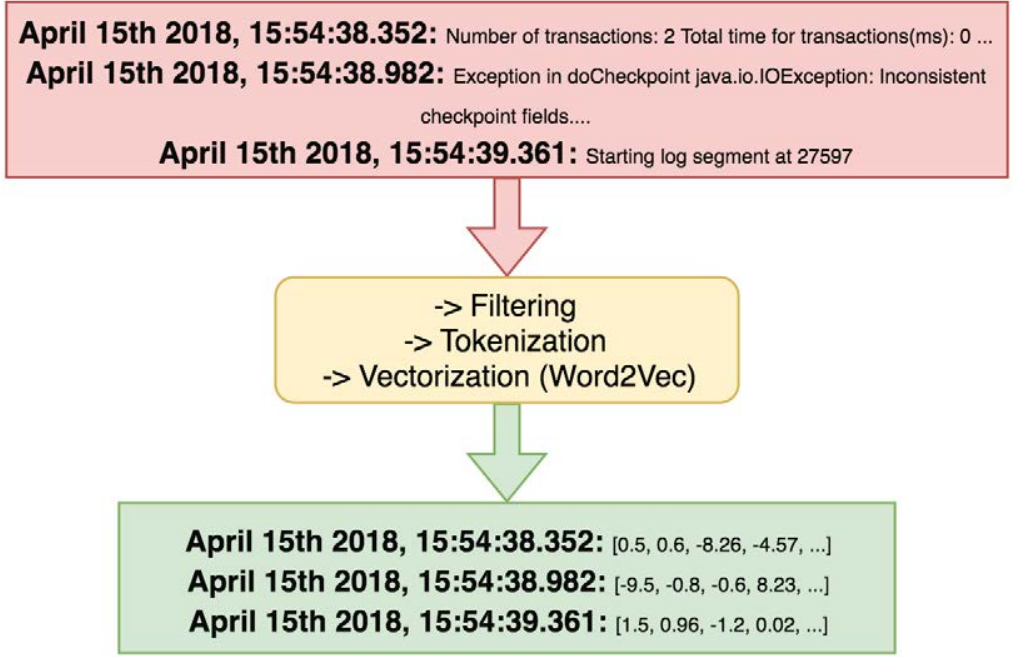

Figure 6.17: An example process of transformation log entries to vectors.

time window are mapped into a vector, and then we aggregate the set of vectors by calculating the average. This resulting vector is added as an attribute, representing the information contained for that log.

The scheme illustrating the whole process is presented in Figure 6.17. The proposed log processing algorithm does not need much configuration work. We only need to adjust a time window size.

\subsubsection{Vector context}

Remember that a context allows to compare two attributes of the same type. Establishing the similarity between two vectors is straightforward through the calculation of the cosine between the two vectors.

$$
\cos (\boldsymbol{x}, \boldsymbol{y})=\frac{\boldsymbol{x} \cdot \boldsymbol{y}}{\|\boldsymbol{x}\| \cdot\|\boldsymbol{y}\|}
$$

In this way, we can compare logs through the vectors created by the Word2Vec process.

\subsubsection{Using metrics distribution for automatic weighting of node at- tributes}

The distribution of values for a metric indicates how different or uncommon a value is. Therefore, it makes sense to use these rare values as a way to change the importance 


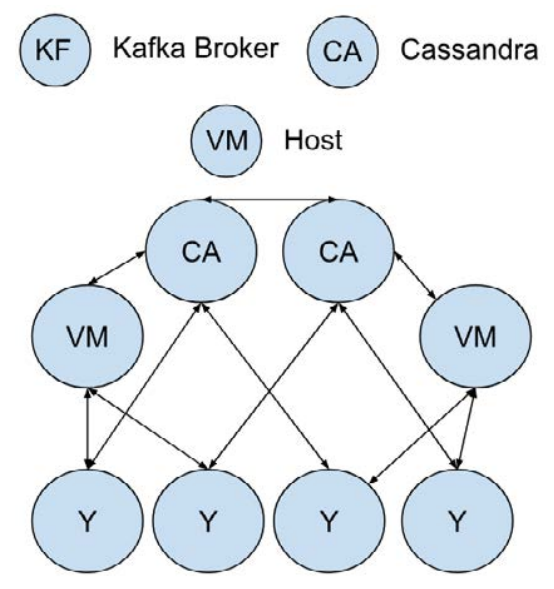

Figure 6.18: The Cassandra architecture

or weight of an attribute. In fact, this follows the same reasoning as a human expert, in which a value that has never been seen before would be a cause of concern. This contributes to knowledge transfer as the weights are automatically set, depending on the values that are registered for an specific scenario.

In this case, we define weights which are proportional to the deviation of the usual value for an attribute. We do so by using the normal distribution $X \sim \mathcal{N}\left(\mu, \sigma^{2}\right)$. The weight of a numerical attribute that is proportional to the deviation of a metric value $a$ is defined as $w(a)=\frac{|a-\mu|}{\sigma}$

\subsubsection{Measuring similarity from metric distributions}

Based on the metric distribution we can also establish how similar are two values of the same attribute. By calculating the cumulative distribution function (CDF) and its parameters, we can define the a similarity function as the formula similarity $=$ 1 -distance where distance is the difference between the $C D F$ values of the attributes. The calculation of distance will vary depending on the type of distribution for that particular metric. For instance, given two numerical attributes from two different graphs $a_{1}, a_{2}$, following a normal distribution $X \sim \mathcal{N}\left(\mu, \sigma^{2}\right)$, their similarity is provided by the formula similarity $=1-\left|\phi_{\mu, \sigma^{2}}\left(a_{1}\right)-\phi_{\mu, \sigma^{2}}\left(a_{2}\right)\right|$ where $\phi_{\mu, \sigma^{2}}$ is the CDF of the normal distribution. 


\subsubsection{Evaluating the cross system diagnostic}

In this section we evaluate the cross system diagnostics. We introduce a new Cassandra (83) scenario. Cassandra is a wide column NoSQL database that is completely decentralised. Nodes exchange information between each other through the use of the Gossip protocol. It can easily scale and has been proved to work in deployments up to 100.000 nodes. Its simplified architecture is depicted in Figure 6.18. In particular, we use 5 Cassandra instances that are going to be continuously queried by 10 containers with Yahoo Cloud Service Benchmark installed. The experimental environment used is the same as in Section 6.4.3 with a DCOS cluster of 8 VM's in Grid'5000.

Regarding the failures we use again stress-ng to stress the CPU, the disk and the network resources of the hosts. We also use docker pause to simulate the temporary lost of connection by a container. The anomalies are injected six times each, in one random element of the architecture for 120 seconds. This is done in both scenarios. The objective is to use the library of anomalous patterns generated by injecting failures in the Kafka architecture to diagnose the same type of failure in Cassandra and vice versa. In this way we can prove that the cross-system diagnostic works by transferring the knowledge from one scenario to the other. We add all the previously mentioned features to the graph similarity engine: embedding the $\log$ of the container as an attribute, automatic weight calculation and comparing metrics using its distribution. The results are presented in Figure 6.19. The average f1-score is 0.77 when using Cassandra as a source system and Kafka as a target one. In the opposite direction the result is 0.76 . This means that most of the knowledge acquired in one scenario can be reused to diagnose the other. It also shows the symmetric properties of the similarity function. The small difference of 0.01 comes from the task of finding the nearest graph, which is not always symmetric as it depends on the patterns that are inserted in the library at a given point in time.

\subsection{Conclusions: graphs are a suitable model for microser- vices}

Throughout this chapter, we have explained why graphs are a good fit to represent the state of microservice architectures. By leveraging graph similarity techniques, we can compare new anomalies that happen in the system with a library of previously 


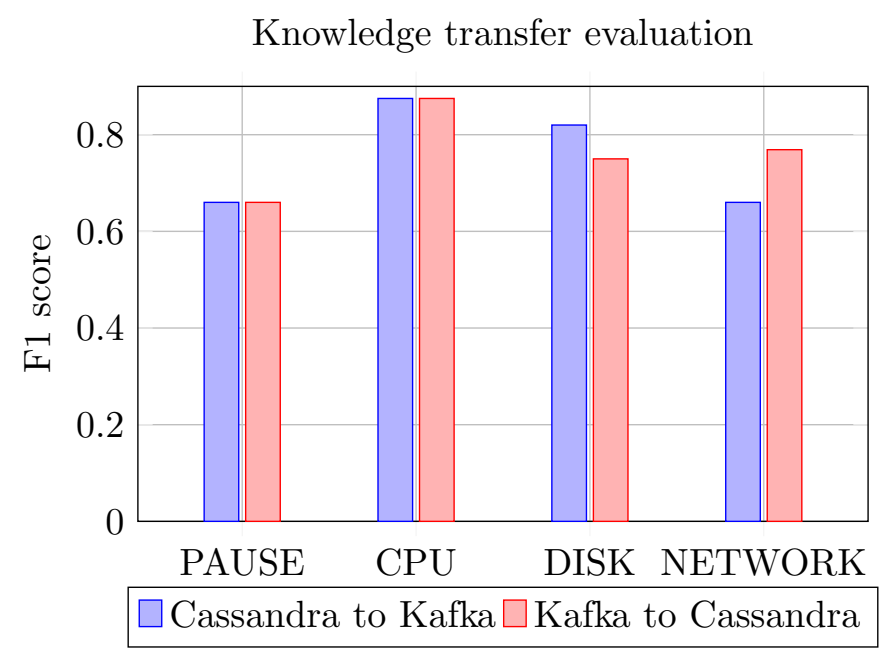

Figure 6.19: F1 scores for the cross-system diagnostics system.

diagnosed anomalous graphs. The root cause analysis will then match the incoming anomaly with the most similar one from the library. The evaluation shows how, through the incorporation of expert knowledge from the user, we are able to accurately match past anomalous situation with current ones, facilitating decision making when maintaining the system. The model is easily interpretable since all the connections and elements can be depicted as a topology map with its edges and nodes. It can also explain why the model makes a given decision, as nodes are matched with their most similar counterpart in the other graph, providing to the user a direct mapping between the anomalous situation and the pattern. Additionally, and since we extract a subgraph that originates from the anomalous node, we are able to include the context surrounding the anomaly by including its neighbours. This is specially important with architectures that have several elements interacting between each other, such as load balancing or master-slave architectures, resulting in better precision than other approaches. Finally, it is able to take inputs from the user in the form of weights, enabling the inclusion of expert knowledge in the domain. This facilitates the human-in-the-loop approach that we wanted to achieve in our framework.

Another feature we have shown is that graphs facilitate knowledge transfer by including semantic representations of general domain knowledge in the form of taxonomies or ontologies. In fact, these graphs could be enriched with additional semantic information to build knowledge graphs representing the state of the system for a given time 
window. This knowledge transfer is further improved by using new features, such as the automatic tuning of weights or comparing cumulative distribution functions and log messages. We have demonstrated the validity of this approach, by transferring the knowledge acquired from observing one technology to troubleshoot a different one.

Containerisation is one of the enabling technologies in Fog computing. The graphbased approach explored in this chapter, can facilitate the representation of microservices due to its modular design and easy interpretability. This approach can also be applied for RCA in the IoT devices, where the heterogeneity of the devices and communication protocols can be embedded into the graph through the attributes and the different contexts available. For instance the different wireless communication technologies between devices (ZigBee, Bluetooth, IEEE 802.15.4 antennas, etc.) can be included as attributes in the edges. There is a great range of possibilities for the graph approach and with these experiments we validated its accuracy. This method is yet another addition to our decision support framework for Fog computing. 


\section{Chapter 7}

\section{Optimising task parallelisation in Big Data analytics}

So far we have described tools for monitoring decentralised infrastructures, such as Fog computing. We have also developed models that perform root cause analysis on microservice architectures, which is one of the key technologies used to run the Fog middleware. Coming back to the concept of the hierarchical structure of the Fog, we will focus now on the top part of the tree, which produces the final intelligence expected from the data gathered from the sensing layer at the bottom. This top part, is made up of a series of Big Data technologies that are able to ingest, store and process all this deluge of information. Same as with other technologies of the ensemble, we need to make sure they are running in an optimal way, as well as facilitating to the user their configuration and maintenance.

In this chapter, we describe the design and implementation of a machine learning based method that recommends an optimal task parallelisation for Big Data workloads. The objective is to have a system able to choose the best parallelisation for the analytic workloads at the Cloud level of the Fog. In particular, we focus on an in-memory analytics engine such as Apache Spark, showing through experimentation how a proper parallelisation can optimise the process in both cost and duration. The content of this chapter is based on the article "Using machine learning to optimize parallelism in big data applications", published in Future Generation Computer Systems Journal 2018 (59). 


\subsection{Task parallelisation with Spark and YARN}

In order to understand why task parallelisation is so important we first need to explain how data processing tasks are distributed across the cluster. Spark is used very often together with a cluster manager such as YARN (139), which keeps track of the resources available in the different machines and allocates the workload depending on a defined strategy. This approach decouples the programming model from the resource management in the cluster. The execution logic is left to the framework (e.g, Spark) and YARN controls the CPU and memory resources in the nodes. In the following two subsections we will explain more in detail how YARN allocates tasks and the parameters and execution flow specific for Apache Spark.

\subsubsection{Resource allocation in YARN}

An application in YARN is coordinated by a unit called the Application Master (AM). $\mathrm{AM}$ is responsible for allocating resources, taking care of task faults and managing the execution flow. We can consider the AM as the agent that negotiates with the manager to get the resources needed by the application. In response to this request, resources are allocated for the application in the form of containers in each machine. The last entity involved is the Node Manager (NM). It is responsible for setting up the container's environment, copying any dependencies to the node and evicting any containers if needed.

The number of available resources can be seen as a two dimensional space formed by memory and cores that are allocated to containers. Both the MB and cores available in each node are configured through the node manager properties: yarn.nodemanager.resource.memory-mb and yarn.nodemanager. resource.cpu-vcores respectively. An important aspect to take into account is how the scheduler considers these resources through the ResourceCalculator. The default option, DefaultResourceCalculator, only considers memory when allocating containers while DominantResourceCalculator, takes into account both dimensions and make decisions based on what is the dominant or bottleneck resource (50). Since CPU is an elastic resource and we do not want to have a limit on it, we will use the DefaultResourceCalculator for YARN in our experiments. By doing this, we will be able to scale up or down the number of tasks running without reaching a CPU usage limit. 


\subsubsection{Spark execution flow and application parameters}

Spark is based on a concept called resilient distributed dataset (RDDs). RDDs are immutable resilient distributed collections of data structures partitioned across nodes that can reside in memory or disk. These records of data can be manipulated through transformations (e.g map or filter). A RDD is lazy in the way that will only be computed when an action is invoked (e.g count number of lines, save as a text file). A Spark application is implemented as a series of actions on these RDDs. When we execute an action over a RDD, a job triggers. Spark then formulates an execution DirectedAcylic-Graph (DAG) whose nodes will represent stages. These stages are composed of a number of tasks that will run in parallel over chunks of our input data, similar to the MapReduce platform. In Figure 7.1 we can see a sample DAG that represents a WordCount application.

When we launch a Spark application, an application master is created. This AM asks the resource manager, YARN in our case, to start the containers in the different machines of the cluster. These containers are also called executors in the Spark framework. The request consists of number of executors, number of cores per executor and memory per executor. We can provide these values through the --num-executors option together with the parameters spark.executor.cores and spark.executor.memory. One unit of spark. executor.cores translates into one task slot. Spark offers a way of dynamically asking for additional executors in the cluster as long as an application has tasks backlogged and resources available. Also executors can be released by an application if it does not have any running tasks. This feature is called dynamic allocation (1) and it turns into better resource utilization. We use this feature in all of our experiments. This means that we do not have to specify the number of executors for an application as Spark will launch the proper number, based on the available resources. Our final target is to find a combination for spark. executor.memory and spark. executor.cores to launch an optimal number of parallel tasks in terms of performance.

\subsection{Studying the impact of parallelism}

The possibility of splitting data into chunks and processing each part in different machines in a divide and conquer manner makes it possible to analyse big amounts of 


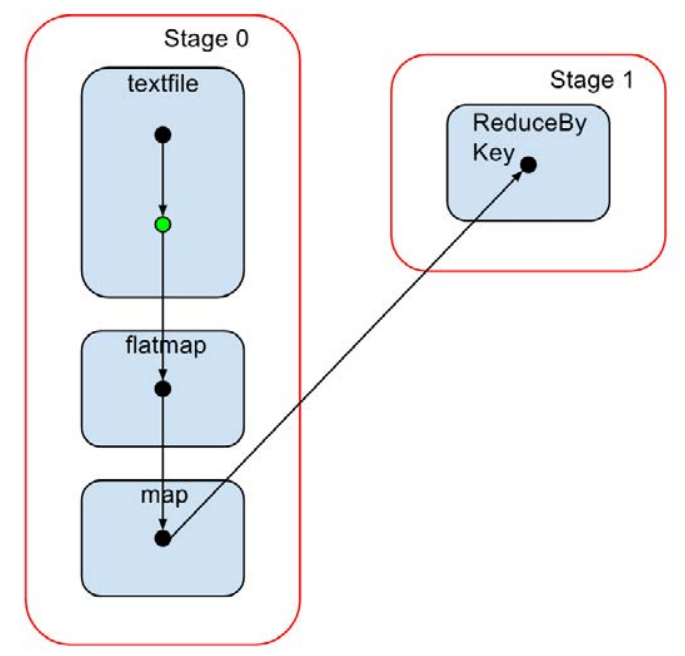

Figure 7.1: Example DAG for WordCount

data in seconds. In Spark, this parallelism is achieved through tasks that run inside executors across the cluster. Users need to choose the memory size and the number of tasks running inside each executor. By default, Spark considers that each executor size will be of $1 \mathrm{~GB}$ with one task running inside. This rule of thumb is not optimal, since each application is going to have different requirements, leading to wastage of resources, long running times and possible failures in the execution among other problems.

We explore these effects in the following experiments. We deployed Spark in four nodes of the Taurus cluster in the Grid 5000 testbed (14). We choose this cluster because we can also evaluate the power consumption through watt-meters in each machine (2). These machines have two processors Intel Xeon E5-2630 with six cores each, 32GB of memory, 10 Gigabit Ethernet connection and two hard disks of 300 GB. The operating system installed in the nodes is Debian 3.2.68, and the software versions are Hadoop 2.6.0 and Spark 1.6.2. We configure the Hadoop cluster with four nodes working as datanodes, one of them as master node and the other three as nodemanagers. Each nodemanager is going to have $28 \mathrm{~GB}$ of memory available for containers. We set the value of vcores to the same number as physical cores. Note however that we are using all the default settings for YARN and by default the resource calculator is going to be DefaultResourceCalculator (3). This means that only memory is going to be taken into account when deciding if there are enough resources for a given application. HDFS is used as the parallel filesystem where we read the files. We intend to illustrate the 


\begin{tabular}{|c|c|}
\hline spark.executor.memory & spark.executor.cores \\
\hline $512 \mathrm{~m}$ & 1 \\
\hline $1 \mathrm{~g}$ & 1 \\
\hline $2 \mathrm{~g}$ & 1 \\
\hline $2 \mathrm{~g}$ & 2 \\
\hline $2 \mathrm{~g}$ & 3 \\
\hline $3 \mathrm{~g}$ & 1 \\
\hline $3 \mathrm{~g}$ & 3 \\
\hline $3 \mathrm{~g}$ & 4 \\
\hline $4 \mathrm{~g}$ & 1 \\
\hline $4 \mathrm{~g}$ & 3 \\
\hline $4 \mathrm{~g}$ & 6 \\
\hline $6 \mathrm{~g}$ & 1 \\
\hline
\end{tabular}

Table 7.1: Combinations of Spark parameters used for the experiments

difference in level of parallelism for applications that have varied requirements in terms of I/O, CPU and memory. To this end, we utilize one commonly used application which is CPU intensive, like kMeans and another common one that is intensive in consumption of I/O, CPU and memory, like PageRank.

Our objective is to see what is the effect of the number of concurrently running tasks on the performance of an application. To do that, we are going to try different combinations of spark.executor.memory and spark. executor.cores, as seen in Table 7.1. We choose these values to consider different scenarios including many small JVM's with one task each, few JVM's with many tasks or big JVM's which only execute one task.

In Figure 7.2, we can see the time and energy it takes to run a kMeans application in the cluster with the default, best and worst configuration. We can observe several effects in both energy and duration for each configuration. Firstly, we observe that the best configuration is $4 \mathrm{~GB}$ and 6 spark.executor.cores. Since kMeans only shuffles a small amount of data, the memory pressure and I/O per task are low, allowing this configuration to run a higher number of tasks concurrently and reducing execution times. Additionally, we can see that the worst configuration is the one with $512 \mathrm{MB}$ and 1 spark.executor.core. Spark allows us to store data in memory for iterative 


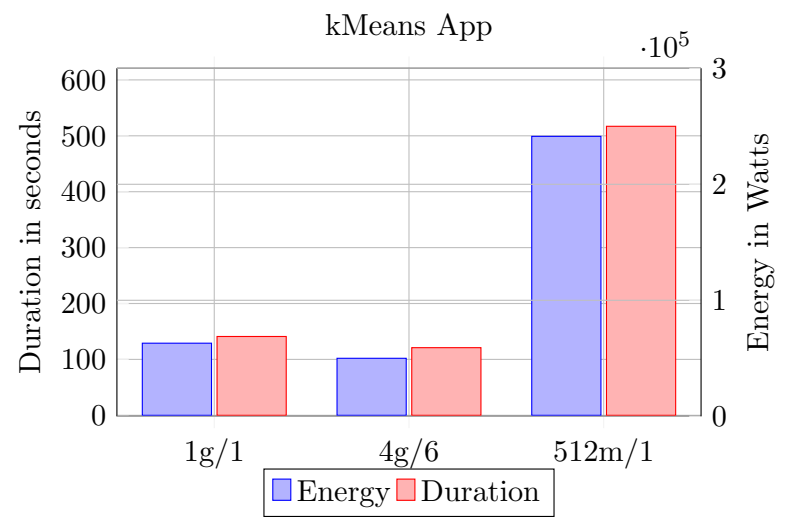

Figure 7.2: Run time in seconds and power consumption in watts for a kMeans App.

The default, best and worst configurations are depicted

workloads like kMeans. Since spark.executor.memory is sized as 512MB, the system does not have enough space to cache data blocks into the executors. This means that in later stages we will have to read that block from disk, incurring in additional latency, specially if that block is in another rack.

The other example is Page Rank, a graph application. Its DAG involves many stages and the configuration will have a great impact on the application performance, as we can see in Figure 7.3. There is a difference of almost 14 minutes between the best execution and the worst one. In comparison to kMeans, PageRank caches data into memory that grows with every iteration, filling the heap space often. It also shuffles gigabytes of data that have to be sent through the network and buffered in memory space. The best configuration is the $2 \mathrm{~g}$ and 2 spark.executor.cores configuration since it provides the best balance between memory space and number of concurrent tasks. For the default one, the executors are not able to meet the memory requirements and the application crashed with an out of memory error. Also, we observed that for the $6 \mathrm{~g}$ and 1 spark. executor.cores, the parallelism is really poor with only a few tasks running concurrently in each node.

We can draw the conclusion that setting the right level of parallelism does have an impact on big data applications in terms of time and energy. The degree of this impact varies depending on the application. For PageRank it can prevent the application from crashing while for kMeans it is only a small gain. Even so, this moderate benefit may have a large impact in organizations that run the same jobs repeatedly. Avoiding 


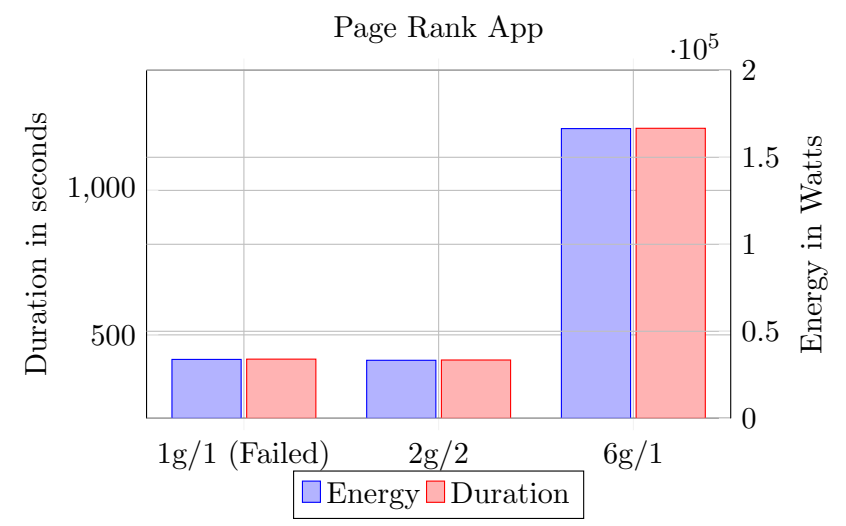

Figure 7.3: Run time in seconds and power consumption in watts for a Page Rank App.

The default, best and worst configurations are depicted

bad configurations is equally important. Providing a recommendation to the user can help him to avoid out of memory errors, suboptimal running times and wasting cluster resources through constant trial and error runs.

\subsection{Model to find the optimal level of parallelism}

As we have seen in the previous section, setting the right level of parallelism can be done for each application through its spark.executor.memory and spark.executor.cores parameters. These values are supposed to be entered by the user and are then used by the resource manager to allocate JVM's. However, clusters are often seen as blackboxes where it is difficult for the user to perform this process. Our objective is to propose a model to facilitate this task. Several challenges need to be tackled to achieve this objective:

- The act of setting these parallelization parameters cannot be detached from the status of the cluster: Normally, the user set some parameters without considering the memory and cores available at that moment in the cluster. However, the problem of setting the right executor size is highly dependent on the memory available in each node. We need the current resource status on YARN as a variable when making these decisions.

- Expert knowledge about the application behaviour is needed: This needs monitoring the resources consumed and metrics of both the application plus the 
system side.

- We have to build a model that adapts to the environment we are using: Different machines and technologies give room to different configurations and parallelism setting.

- We have to be able to apply the experience of previous observations to new executions: This will simplify the tuning part to the user every time he launches a new application.

To solve these problems we leverage the machine learning techniques. Following the same approach as with our graph-based RCA method for microservices, we are going to monitor and gather data about the infrastructure, pre-process and integrate the data, train the AI model and use it to choose the best option in the decision space. In the following subsections we will explain the details of how we gather the metrics, how we build a dataset that can be used to solve this problem and the methodology used to make the recommendations.

\subsubsection{Gathering the metrics and building new features}

Predictive machine learning algorithms need a dataset with a vector of features $x_{1}, x_{2}, \ldots x_{n}$ as an input. In this section we will explain which features we use to characterize Spark applications. They can be classified in three groups:

- Parallelism features: They are metrics that describe the concurrent tasks running on the machine and the cluster. Please note that these are different from the parallelization parameters. The parallelization parameters are the spark.executor.cores and spark . executor .memory parameters, which are set up by the users, while the parallelism features describe the effect of these parameters on the applications. We elaborate upon these features in Subsection 7.3.1.1.

- Application features: These are the metrics that describe the status of execution in Spark. Examples of these features are number of tasks launched, bytes shuffled or the proportion of data that was cached. 
- System features: These are the metrics that represent the load of the machines involved. Examples include CPU load, number of I/O operations or context switches.

In the following subsections, we will explain how we monitor these metrics and how we build new additional metrics, like the number of tasks waves spanned by a given configuration or the RDD persistence capabilities of Spark.

\subsubsection{Parallelism features}

A Spark stage spans the number of tasks that are equal to the number of partitions of our RDD. This number can be set statically through instructions in the Spark API, or it is defined depending on the filesystem from where we are reading the data. In our case, we are going to assume that HDFS is our parallel file system of choice. The number of partitions when reading a file is given by the number of HDFS blocks of that file. Since we want to configure the workload parallelism at launch time, depending on variable factors, like the resources available in YARN, we will not consider the API approach and focus on the HDFS one. The goal is to calculate, before launching an application, the number of executors, tasks per executors and tasks per machine that will run with a given configuration and YARN memory available. These metrics will be later used by the model to predict the optimal configuration. To formulate this, we are going to use the calculations followed by YARN to size the containers. We need the following variables:

- Filesize $\left(f_{\text {size }}\right)$ : The number of tasks spanned by Spark is given by the file size of the input file.

- dfs.block.size $\left(b_{h d f s}\right)$ : An HDFS parameter that specifies the size of the block in the filesystem. We keep the default value of $128 \mathrm{MB}$.

- yarn.scheduler.minimum.allocation-mb ( min $\left._{y a r n}\right)$ : The minimum size for any container request. If we make requests under this threshold it will be set to this value. We use its default value of 1024MB.

- spark.executor.memory $\left(\right.$ mem $\left._{\text {spark }}\right)$ : The size of the memory to be used by the executors. This is not the final size of the YARN container, as we will explain later, since some off-heap memory is needed. 
- spark.executor.cores $\left(\right.$ cor $\left._{\text {spark }}\right)$ : The number of tasks that will run inside each executor.

- yarn.nodemanager.resource.memory-mb parameter $\left(\right.$ mem $\left._{\text {node }}\right)$ : This sets the amount of memory that YARN will have availabe in each node.

- spark.yarn.executor.memoryOverhead $\left(\right.$ over $\left._{y a r n}\right)$ : The amount of available offheap memory. By default, its value its 384 .

- Total available nodes $\left(N_{\text {nodes }}\right)$ : This represents the number of nodes where we can run our executors.

The size of an executor is given by:

$$
\text { Size }_{\text {exec }}=\text { mem }_{\text {spark }}+\max \left(\text { over }_{\text {yarn }}, \text { mem }_{\text {spark }} * 0.10\right)
$$

The 0.10 is a fixed factor in Spark and it is used to reserve a fraction of memory for the off-heap memory. We then round up the size of the executor to the nearest multiple of yarn min $_{y a r n}$ to get the final size. For example, if we get a Size exec $=1408$, we will round up to two units of min $_{y a r n}$ resulting in $S i z e_{\text {exec }}=2048$. Now we can calculate the number of executors in each node as $N_{\text {exec }}=\left\lfloor\right.$ mem $_{\text {node }} / S_{\text {Size }}$ exec $\rfloor$ and the number of task slots in each node as slots $s_{\text {node }}=\left\lfloor N_{\text {exec }} *\right.$ cor $\left._{\text {spark }}\right\rfloor$. Consequently, the total number of slots in the cluster are slots $_{\text {cluster }}=\left\lfloor\right.$ slots $\left._{\text {node }} * N_{\text {nodes }}\right\rfloor$.

Finally, we also want to know the number of waves that will be needed to run all the tasks of that stage. By waves we mean the number of times a tasks slot of an executor will be used, as depicted in Figure 7.4. We first calculate the number of tasks that will be needed to process the input data. This is given by $N_{\text {tasks }}=\left\lfloor f_{\text {size }} / b_{h d f s}\right\rfloor$. Then the number of waves is found by dividing this task number between the number of slots in the cluster $N_{\text {waves }}=\left\lfloor N_{\text {tasks }} /\right.$ slots $\left.s_{\text {cluster }}\right\rfloor$. Summarizing all the previous metrics that are useful to us, we get the following set of metrics, which we will call parallelism metrics:

$$
\left\{N_{\text {tasks }}, \text { slots } s_{\text {node }}, \text { slots }_{\text {cluster }}, N_{\text {waves }}, \text { size }_{\text {exec }}, \text { cor }_{\text {spark }}\right\}
$$

These are the metrics that will vary depending on the resources available in YARN and the configuration we choose for the Spark executors and that will help us to model performance in terms of parallelism. 


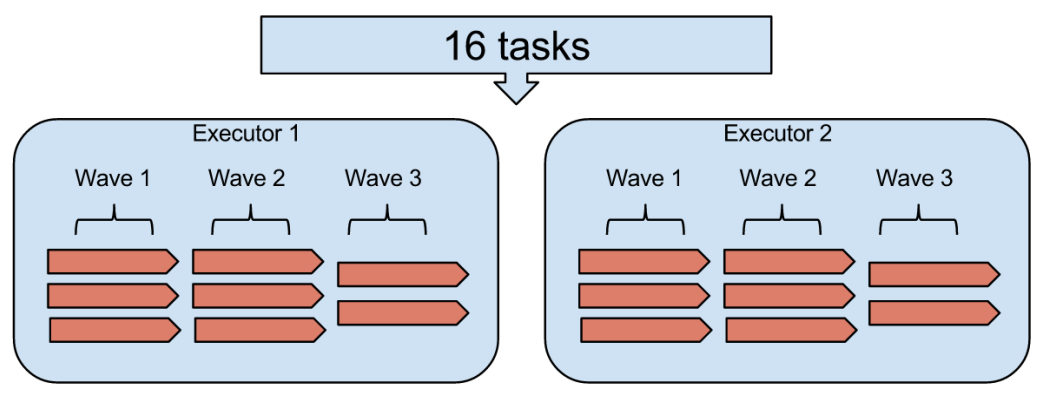

Figure 7.4: A set of waves spanned by two executors with three spark.executor.cores. If we have 16 tasks to be executed then it will take three waves to run them in the cluster

\subsubsection{Application features}

Spark emits an event every time the application performs an action at the task, stage, job and application levels explained in Section 7.1.2. These events include metrics that we capture through Slin 47 a node.js server 48 that stores these metrics in a MongoDB 49 database and aggregates them at task, stage, job and application level in a bottom to up manner. This means that if we aggregate the counters for all the tasks belonging to a stage, we will have the overall metrics for that stage. The same is done at job level, by aggregating the stage metrics and at application level, by aggregating the jobs, as shown in Figure 7.5 .

This however creates an issue. We want to characterize applications, but if we aggregate the metrics for each tasks then we will have very different results depending on the number of tasks launched. As we explained in Section 7.3.1.1, the number of tasks spanned depends on the size of the input file. This is not descriptive as we want to be able to detect similar applications, based on their behaviour and the operations they perform, even if they operate with different file sizes. Let's assume we have some metrics we gathered by monitoring the only stage of the Grep application with 64 tasks. We need these metrics to describe the application, independently of the file size with which it was executed. In this example, if this same stage is launched again in the future with 128 tasks, its metrics have to be similar to the ones of the 64 tasks

\footnotetext{
${ }^{47}$ https://github.com/hammerlab/slim

${ }^{48}$ https://nodejs.org/

${ }^{49}$ https://www.mongodb.com/
} 


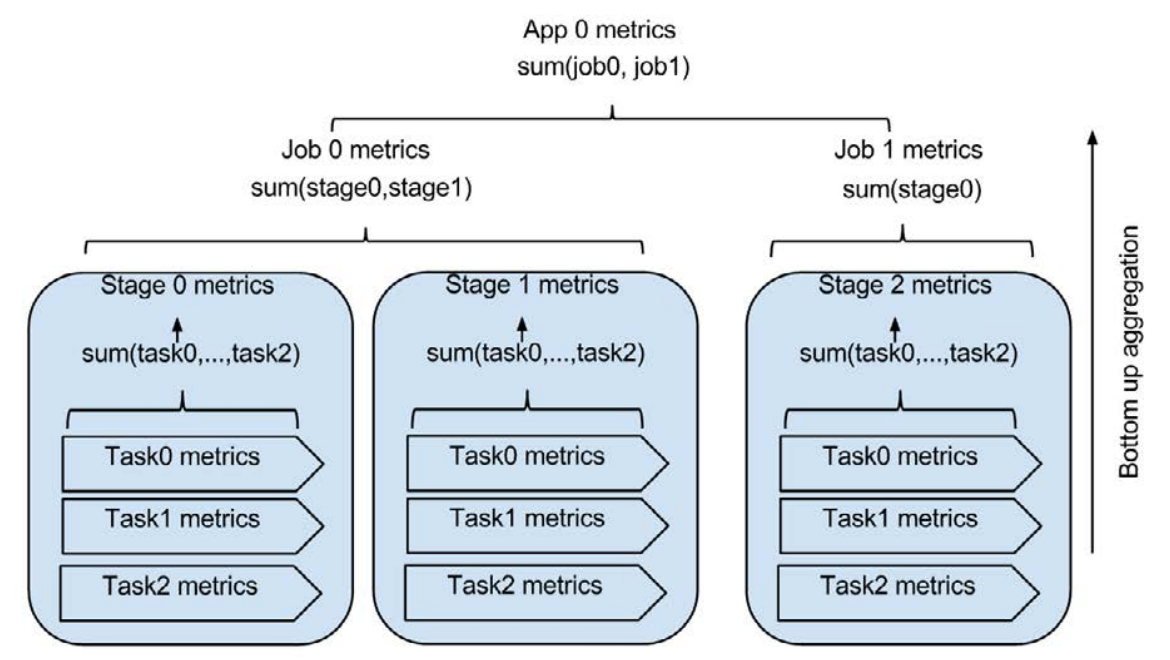

Figure 7.5: Slim bottom-up aggregation. If we have the metrics for the tasks, Slim can aggregate the ones belonging to a stage to calculate that stage metrics. It does the same at the job and application execution level

execution in order to apply what the model learned so far for Grep or similar behaving applications.

To even out these situations, we calculate the mean of the metrics for the tasks of that stage. Figure 7.6 shows that the mean gives us similar metrics independently of the filesize for a stage of Sort. The metrics are normalized from 0 to 1 to be able to plot them together, since they have different scales.

We also define an additional metric at the application level to describe how Spark persists data. We derive a persistence score vector for each stage of an application that describes how much of the total input was read from memory, local disk, HDFS or through the network. For a stage $S$, we have a total number of task $\left(N_{\text {tasks }}\right)$. We count the number of these tasks that read their input from different sources as memory $\left(N_{\text {memory }}\right)$, disk $\left(N_{\text {disk }}\right)$, HDFS $\left(N_{\text {hdfs }}\right)$ and network $N_{\text {network }}$. The vector for that stage $V_{S}$ is:

$$
V_{S}=\left\{\frac{N_{\text {memory }}}{N_{\text {tasks }}}, \frac{N_{\text {disk }}}{N_{\text {tasks }}}, \frac{N_{\text {hdfs }}}{N_{\text {tasks }}}, \frac{N_{\text {network }}}{N_{\text {tasks }}}\right\}
$$

This vector will describe if the stage processes data that is persisted in memory, disk or if it was not on that node at all and had to be read through the network. We also use the HDFS variable to know if the stage reads the data from the distributed file 
Application metrics for different execution sizes

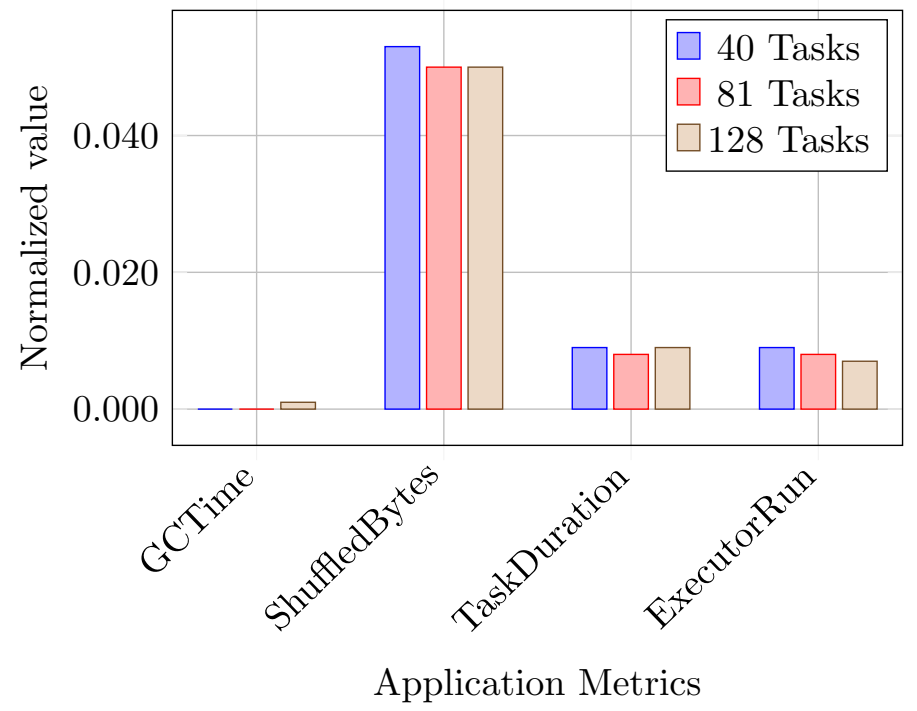

Figure 7.6: Mean application metrics values for a stage of a Sort application with different filesizes. We can see how the mean of the metrics is almost the same for 40,81 and 128 tasks

system. Usually this belongs to the first stage of an application, since it is the moment when we first read data and create the RDD.

An important issue to notice here is that $V_{S}$ can change depending on the execution. Sometimes, Spark will be able to allocate tasks in the same node where the data is persisted. In some other executions, it will not be possible because all slots are occupied or because the system has run out of memory. However, the objective is not to have a detailed description of the number of tasks that read persisted data. Instead, we want to know if that stage persists data at all and how much it was able to. Later on, the model will use this to statistically infer how different configurations can affect a stage that persists data.

\subsubsection{System features}

There are aspects of the workload that cannot be monitored at application level, but at system level, like the time the CPU is waiting for I/O operations or the number of bytes of memory paged in and out. To have a complete and detailed signature of the behaviour of an application, we also include these kinds of metrics. We include here 
features like cpu_usr, bytes paged in/out or bytes sent through the network interface. To capture them, we use GMonE. We developed a plugin that uses Dstat 50 . With this, we obtain a measure per second of the state of the CPU, disk, network and memory of the machines in our cluster.

Since one task will run only in one node, we calculate the mean of the system metrics on the node during the lifespan of that task. This will give us descriptive statistics about the impact of that task at the system level. Then, for each stage we will calculate the mean of the metrics for all of its tasks in a similar bottom up way, as we explained in Section 7.3.1.2. This will represent what happened at the system level while this application was running.

\subsubsection{Building the dataset and training the model}

For each stage executed in our cluster, we get a vector that we use as a data point. We will represent this database of stages as:

$$
\text { stagesDB }=\left\{X_{\text {app }}, X_{\text {system }}, X_{\text {parallelism }}, Y_{\text {duration }}\right\}
$$

where the X's are the features at application (Section 7.3.1.2), system (Section 7.3.1.3) and parallelism level (Section 7.3.1.1) and $Y_{\text {duration }}$ is the duration of that stage or target variable to predict. Now we have to build the dataset that we will use to train the model. We want to answer the following question: If we have a stage with metrics $X_{a p p}$ and $X_{\text {system, }}$, collected under some $X_{\text {parallelism }}$ conditions and its duration was $Y_{\text {duration }}$, what will be the new duration under some different $X_{\text {parallelism }}$ conditions?. We can regress this effect from the data points we have from previous executions. However, we have to take into account that metrics change depending on the original conditions in which that stage was executed. For instance it is not the same executing a stage with executors of $1 \mathrm{~GB}$ and 1 task compared to $3 \mathrm{~GB}$ and 4 tasks. Metrics like garbage collection time will increase if tasks have less memory for their objects. In addition, CPU wait will increase if we have many tasks in the same machine competing for I/O. We can see this effect in Figure 7.7, where the metrics of a stage of a support vector machine implementation in Spark change depending on the number of tasks per node we choose.

\footnotetext{
${ }^{50}$ http://dag.wiee.rs/home-made/dstat/
} 
CPU values and tasks per node

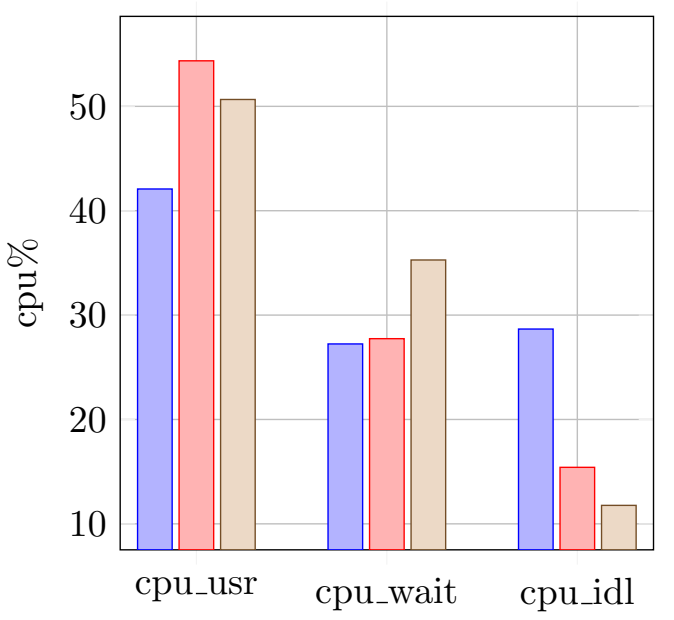

GC and deserialize time values and tasks per node

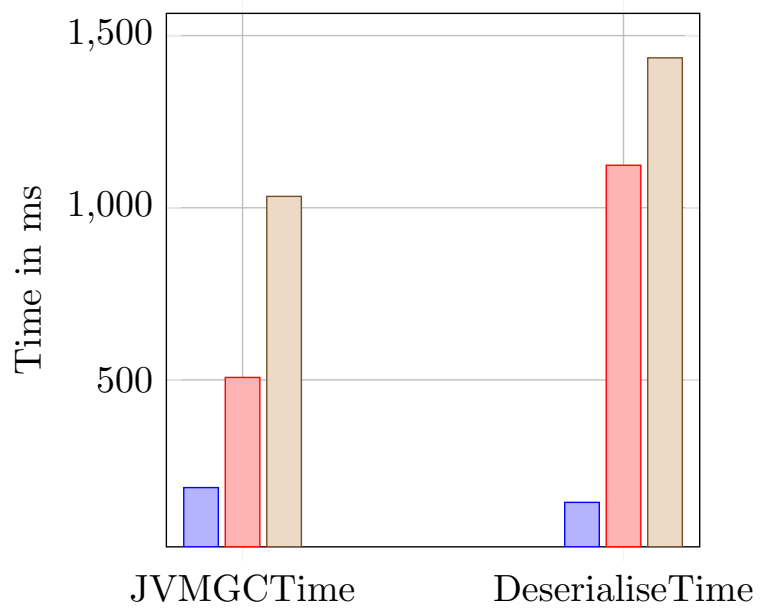

\section{4 tasks $\square \quad \square$ tasks $\square 20$ tasks}

Figure 7.7: Changes in the metrics for a stage of a support vector machine implementation in Spark. Note how the CPU metrics change depending on the number of tasks per node. Also garbage collection and the deserialize time of an stage increases with this number.

To solve this variance in the metrics, we have to include two set of parallelism values in the learning process:

- The parallelism conditions under which the metrics were collected. We will call them $X_{\text {parallelism }_{\text {ref }}}$.

- The new parallelism conditions under which we will run the application. We will call them $X_{\text {parallelism }}$ run .

Now building the dataset is just a matter of performing a cartesian product between the metrics $\left\{X_{a p p}, X_{\text {system }}, X_{\text {parallelism }}\right\}$ of a stage and the $\left\{X_{\text {parallelism }}, Y_{\text {duration }}\right\}$ of all the executions we have for that same stage. For example, let's assume we have two executions of a stage with different configurations like 10 and 4 tasks per node. We also have all their features, including system plus application metrics, and the duration of the stage. The logic behind this cartesian product is: If we have the metrics $\left(X_{10}\right)$ and the duration $\left(Y_{10}\right)$ of an execution with 10 tasks per node (tpn) and we have the metrics $\left(X_{4}\right)$ and the duration $\left(Y_{4}\right)$ of an execution with 4 tpn, then the metrics of the 
10 tasks execution together with a configuration of 4 tasks per node can be correlated with the duration of the 4 tpn execution, creating a new point like:

$$
\left\{X_{10 a p p}, X_{10 \text { system }}, X_{1_{\text {10arallelism }} \text { ref }}, X_{4 \text { parallelism }_{\text {run }}}, Y_{4 \text { duration }}\right\}
$$

Note here that we consider that two stages are the same when they belong to the same application and the same position in the DAG. The algorithm is shown in Algorithm 1.

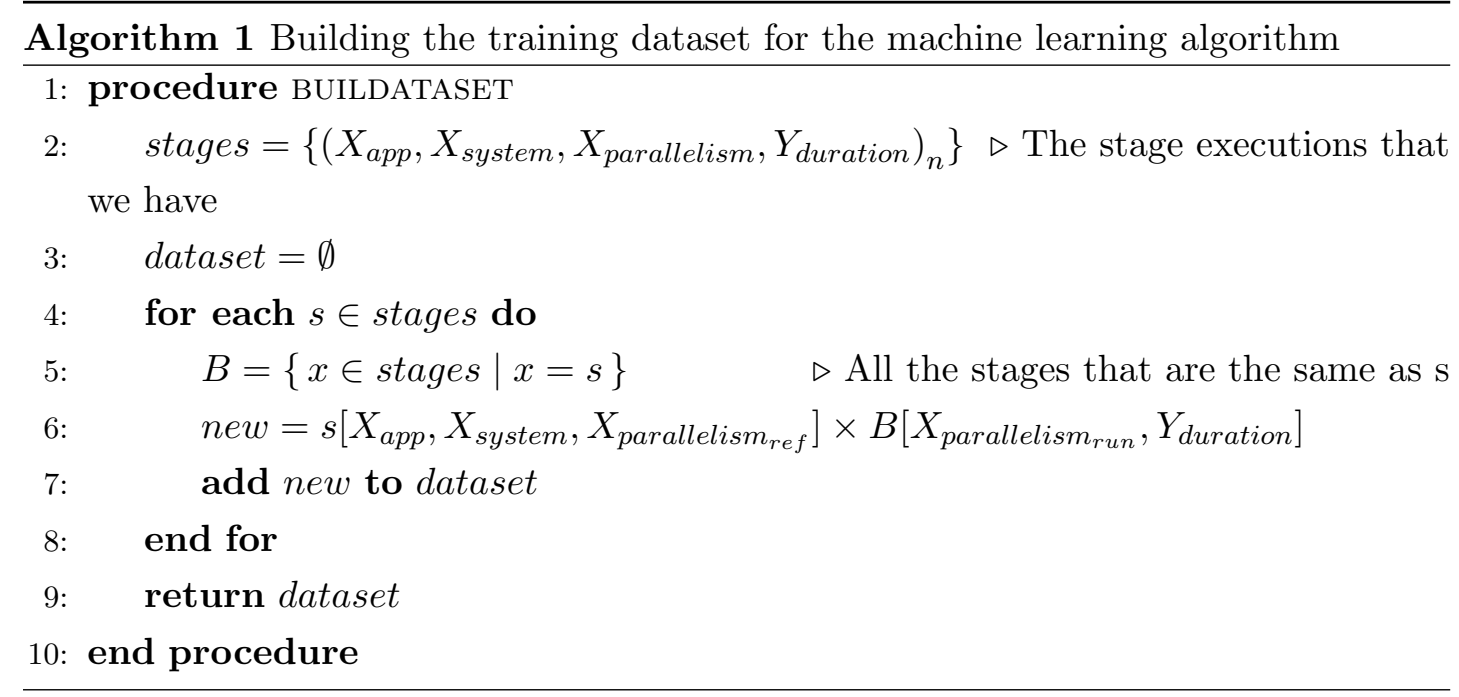

Now we can train the machine learning model with this dataset and check its prediction accuracy. First we have to choose the most accurate implementation. Since this is a regression problem, we try different state-of-the-art regression algorithms: Bayesian Ridge, Linear Regression, SGD Regressor, Lasso, Gradient Boosting Regressor, Support Vector Regression and MLPRegressor, all from the sklearn Python library (107). First of all, we choose these regression algorithms versus other solutions, like Deep Learning, because they are interpretable. Apart from this, the chosen algorithms are easily evaluated with cross validation techniques, as K-fold, Leave P-out or Leave One Group Out, among others. From a practical point of view, these algorithms are available in the scikit-learn package, which provides implementations ready to be used right out of the box. They also allow us to create pipelines where we perform a series of preprocessing steps before training the model. We build a pipeline for each of the previous 
algorithms, where we first standardize the data and then fit the model. Standardization of the data is a common previous step of many machine learning estimators. It is performed for each feature, by removing its mean value, and then scaling it by dividing by its standard deviation. This ensures an equal impact of different variables on the final outcome and not being biased towards variables that have higher or lower numerical values. Then for each pipeline, we perform a ten times cross validation with $3 \mathrm{k}$-folds (79). The results are shown in Figure 7.8. The metric used to evaluate the score is Mean Absolute Error (MAE) (143). It is calculated by averaging the absolute errors between predicted and true values for all the data points. MAE will allow us to evaluate how close the different predictors are to the real values. We do not include SGD Regressor, since its MAE is just too high to compare with the other methods. As we can see, boosted regression trees have the best accuracy amongst the seven methods and also the lowest variance. In addition, decision trees are interpretable. This means that we will be able to quantify the effect of any of the features of the model on the overall performance of the application.

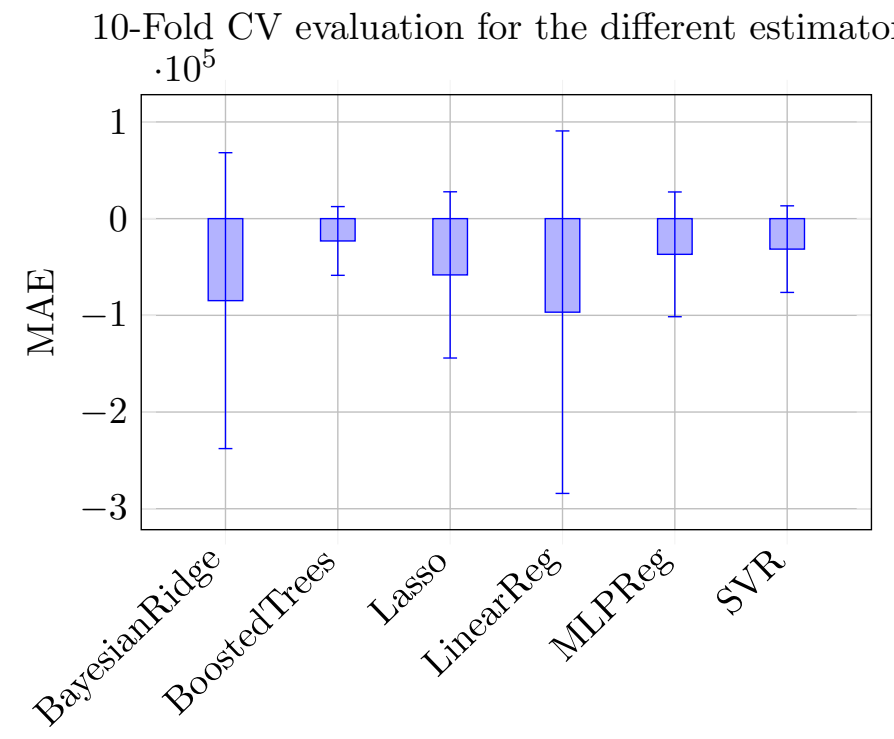

Algorithm

Figure 7.8: $10 \mathrm{CV}$ with $3 \mathrm{~K}$-Fold MAE score for different regression algorithms. GradientBoostingRegressor is the clear winner with a median absolute error of 23 seconds, a maximum of 94 seconds in the last 10th iteration and a minimum of 2.7 seconds for the 3rd iteration 


\subsubsection{Using the model: providing recommendations for applications}

So far, we have only characterized and built our model for stages. An application is a DAG made out of stages. The questions then arises as to how can we provide a parallelization recommendation for the whole application based on the previous explained concepts. Remember that a configuration in Spark is given at application level. Thus, if we set some values for spark.executor.memory and spark.executor.cores, they will be applied to all the stages. If we have a list listofconfs with combinations of different values for these two parameters, and we launch an application with a different combination each time, each execution will have different tasks per node, waves of tasks and memory per task settings. In other words, and following the previously introduced notation, each execution will have different $X_{\text {parallelism }}$ features. The dataset built in Algorithm 1 had data points like:

$$
\left\{X_{\text {app }}, X_{\text {system }}, X_{\text {parallelism }_{\text {ref }}}, X_{\text {parallelism }_{\text {run }}}, Y_{\text {duration }}\right\}
$$

therefore, we can plug in new values for $X_{\text {parallelism }_{\text {run }}}$ by iterating through the different configurations in listof conf $s$, and predict the new $Y_{\text {duration }}$ for the parameters. In addition, the objective is to know the effect of a given configuration on the whole application. So we can perform this process for all the stages that are part of the application and sum the predictions for each configuration. Then we only have to choose as the optimal configuration the one from the list that yields the minimum predicted duration. Algorithm 2 describes this process for a given application and its input data size. The time complexity of the boosted decision trees model, used to make the predictions, is $O(n t r e e * n * d)$, where ntree is the number of trees of the boosted model, $d$ is the depth of those trees and $n$ is the number of data points to be predicted. We have to do this prediction for the nconf configurations of the configuration list, which transforms it into $O(n t r e e * n * d * n c o n f)$. Note that the only term that is variable here is the number of data points $n$, that will grow as we have more executions on our system. The rest will remain constant as they are fixed the moment we train the model and are small numbers. We have to point out that we do not consider stages that launch only one task, since it is trivial that parallelism will not bring any benefit to them. The DAG engine of Spark can also detect that some stages do not have to be recomputed and so they can be skipped. We do not consider these kinds of stages in 
the DAG either since their runtime is always 0. Figure 7.9 shows a graphical depiction of the workflow for a Sort app example.

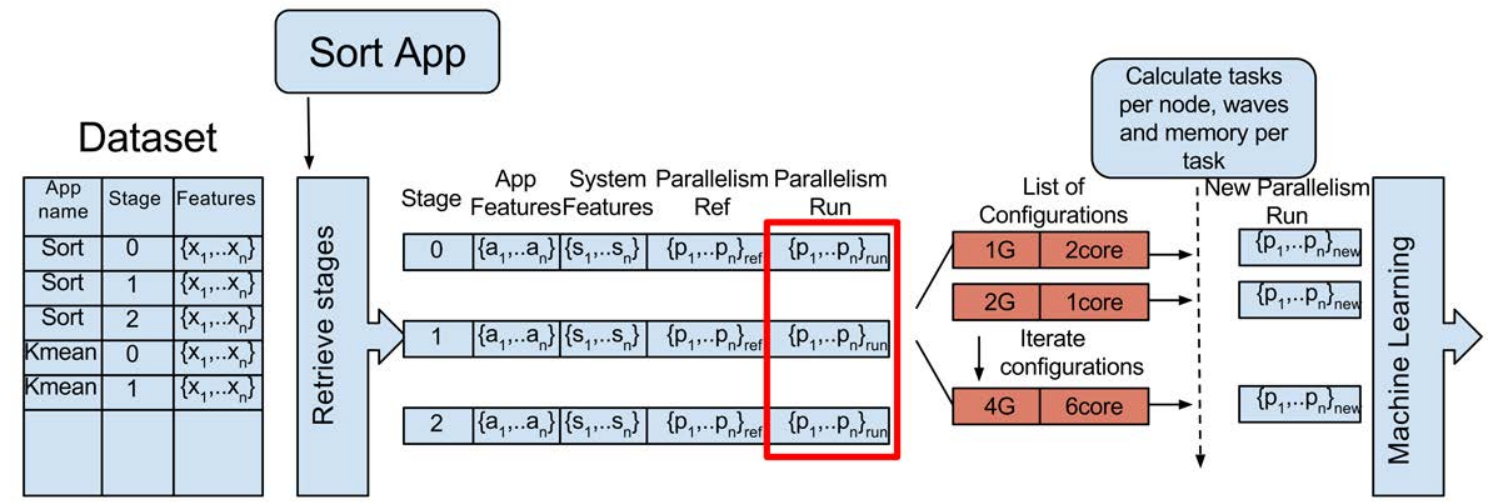

Figure 7.9: An example with a Sort App, where we iterate through a configuration list. Notice the different parallelism features: $\left\{p_{1}, \ldots, p_{n}\right\}_{\text {ref }}$ represent the parallelism conditions under which the metrics were collected, $\left\{p_{1}, \ldots, p_{n}\right\}_{\text {run }}$ the parallelism conditions under which the application run for that execution and $\left\{p_{1}, \ldots, p_{n}\right\}_{n e w}$ the new parallelism conditions that we want to try and the ones we will pass to the machine learning module. The rest of the metrics are kept constant. Then we just have to group by configuration and sum up each of the stages predictions to know which one is best

\subsubsection{Dealing with applications that fail}

When we explained the process by which we predict the best parallelism configuration, we assumed that we have seen at least once all the stages of an application. However, this is not always the case, since some configurations may crash. For example, for a Spark ConnectedComponent application with a filesize of 8GB, the only configurations that were able to complete successfully in our experiments were the ones with $4 \mathrm{~GB}$ and 1 core and $6 \mathrm{~GB}$ and 1 core. The reason behind it is that graph processing applications are resource hungry and iterative by nature. This can put a lot of pressure in the memory management, specially when we cache RDD's like in these kinds of implementations. In this particular example, the $8 \mathrm{~GB}$ file we initially read from HDFS grows to a $17.4 \mathrm{~GB}$ cached RDD in the final stages of the application.

Thus, if we choose an insufficient memory setting, like the default 1GB configuration, the application will not finish successfully and we will not have either the complete number of stages or the metrics for that application. Incomplete information means 


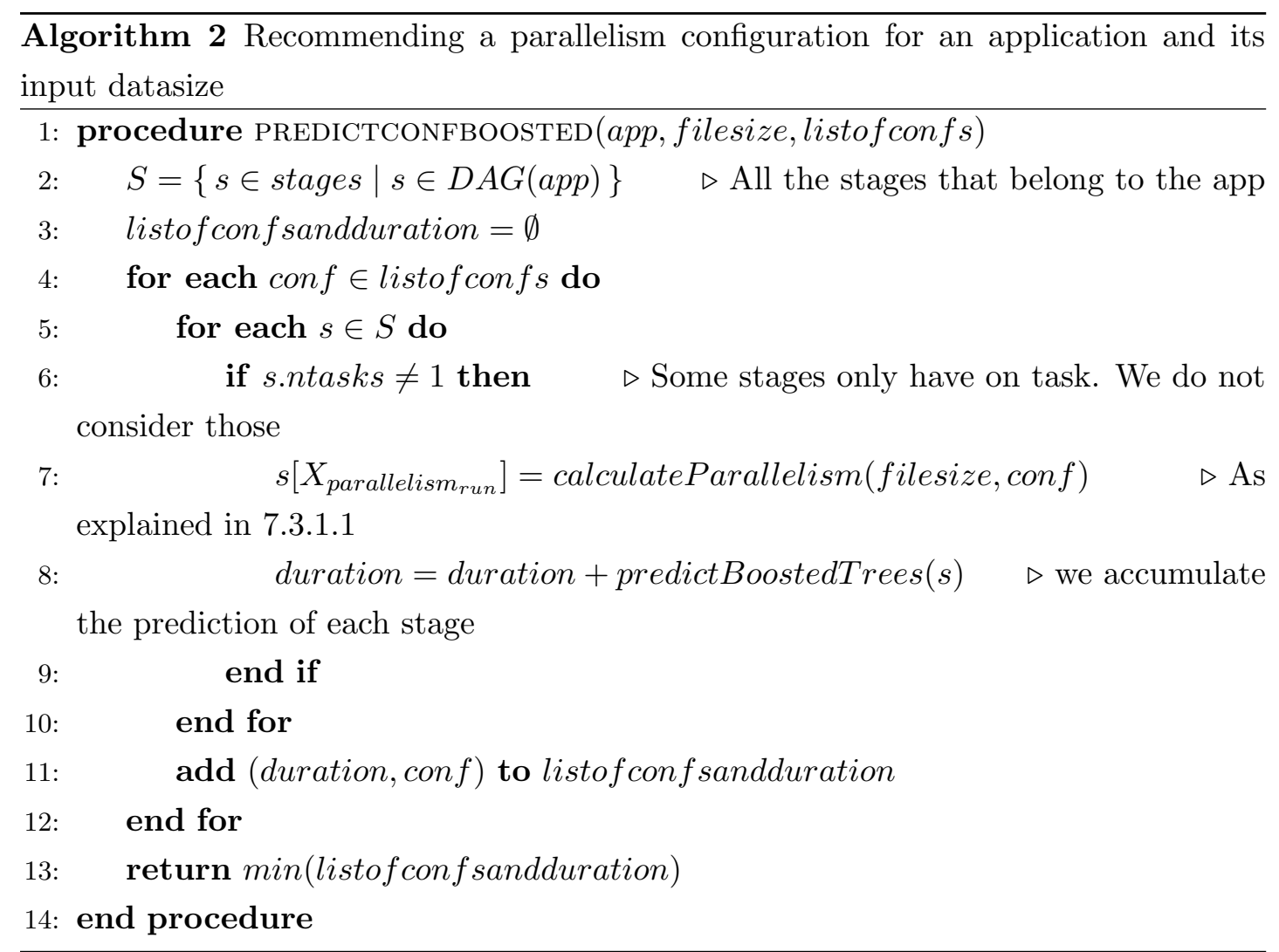

that the next configuration recommended could be non-optimal, drawing us into a loop where the application continuously crashes, as depicted in Figure 7.10.

To solve this, we use the approach explained in Algorithm 3. When a user launches an application, we check if there are any successful executions for it. If we cannot find any then we retrieve the furthest stage the application got to. This can be considered as the point where the execution could not continue. For that stage we find the knearest neighbours from all the stages that we have seen so far. These neighbouring stages belong to a set of applications. Then we follow a conservative approach where we choose the configuration amongst these applications that resulted in the most number of stages completed. The intuition behind it is that a configuration with a high number of completed stages means better stability to the application. With this, we aim to achieve a complete execution of the application and to collect the features of all the stages with it. If the user executes the same application in the future, we can use this complete information together with the standard boosted gradient model to recommend a new 


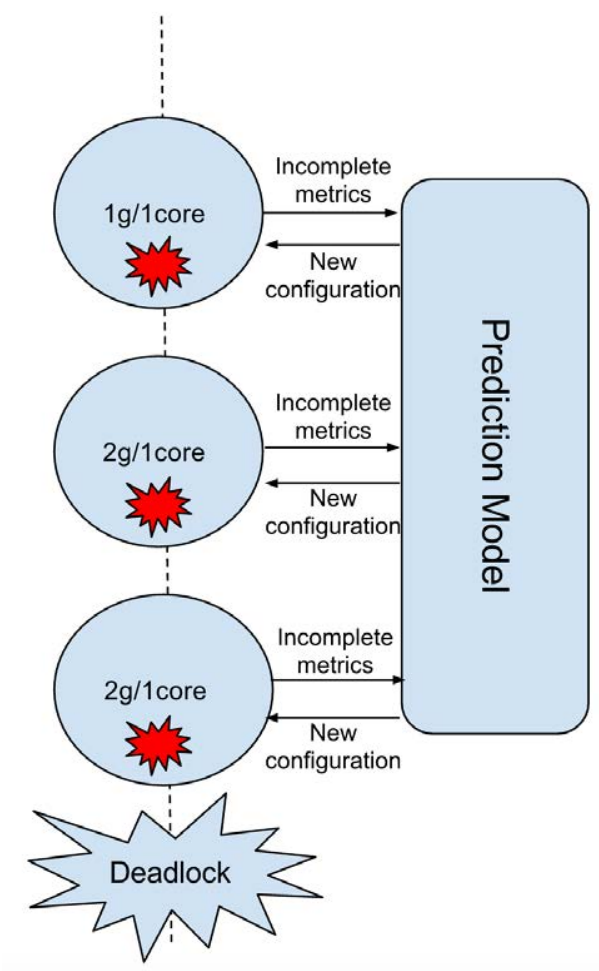

Figure 7.10: A deadlock situation in which the same configuration is recommended continuously. The application crashes with the default configuration. Based on whichever stages executed successfully the next recommendation also crashes. If we do not get past the crashing point the recommendation will always be the same

configuration. By trying different values for the number of $\mathrm{k}$ neighbours, we found that 3 is a good number for this parameter. Going above this value can retrieve too many diverse stages, which could make the application to crash again. Going below it can take us to the same deadlock depicted in Figure 7.10. Remember that the objective is not to find an optimal configuration, but a conservative configuration, which enables a complete execution without failures.

The kNeighbours approach uses a K-D Tree search approach, where the complexity is $O(\log (n))$ with $n$ being the number of data points.

\subsubsection{The recommendation workflow}

Now that we have developed the models with which we make recommendations, we can connect everything together and describe the workflow we follow to find the best 


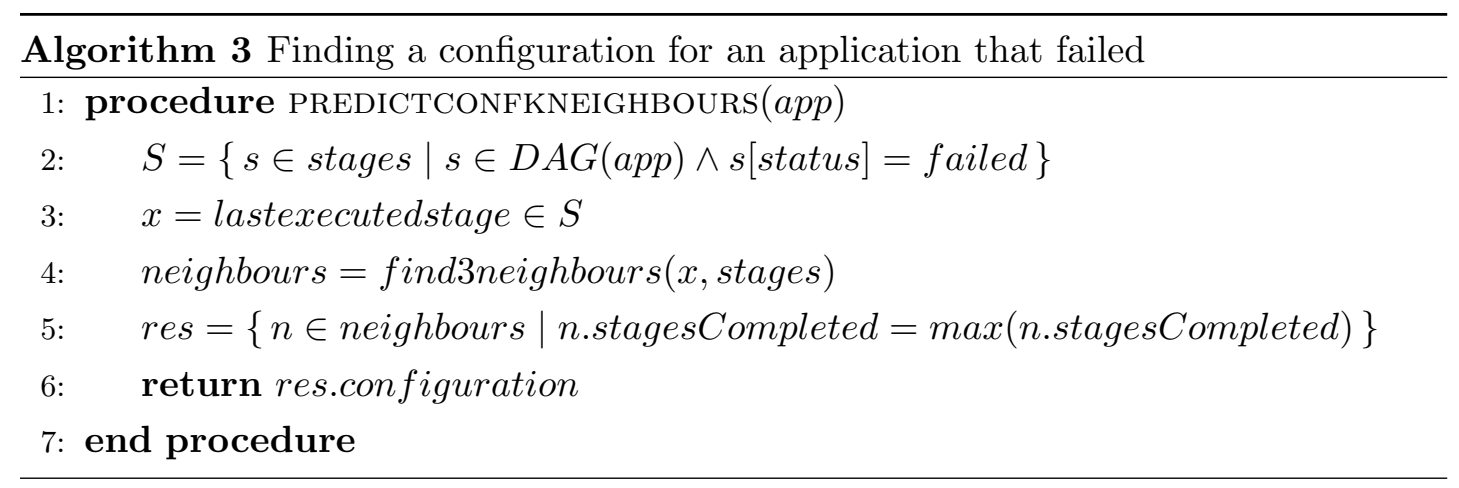

parallelism settings. When a user wants to execute an application, first we check if we have any information of it in our database of previously seen applications. If we do not have it, we execute it with the default configuration. This execution will give us the metrics we need to tune the application in future executions. If we do have information about that application, we check if it completed successfully or not:

- In case it did not, we apply the procedure of Algorithm 3 for crashed executions.

- In case it did, we apply the Algorithm 2 with the boosted regression trees model.

Whatever the case is, the monitoring system adds the metrics for that run to our database so they can be applied for future executions. Applications in a cluster are recurring (23). This means that the precision of the model will increase with the time since we will have more data points. We assume the machine learning algorithm has been trained beforehand and we consider out of the scope of this paper and as future work, when and how to retrain it. A final algorithm for the process is depicted in Algorithm 4.

\subsection{Evaluation}

We perform an offline evaluation of the model with the traces we got from a series of experiments in Grid'5000.

Cluster setup: Six nodes of the Adonis cluster in the Grid'5000 testbed. These machines have two processors Intel Xeon E5520 with four cores each, 24GB of memory, 1 Gigabit Ethernet and 1 card InfiniBand 40G and a single SATA hard disk of 250 GB. The operating system installed in the nodes is Debian 3.2.68, and the software versions 


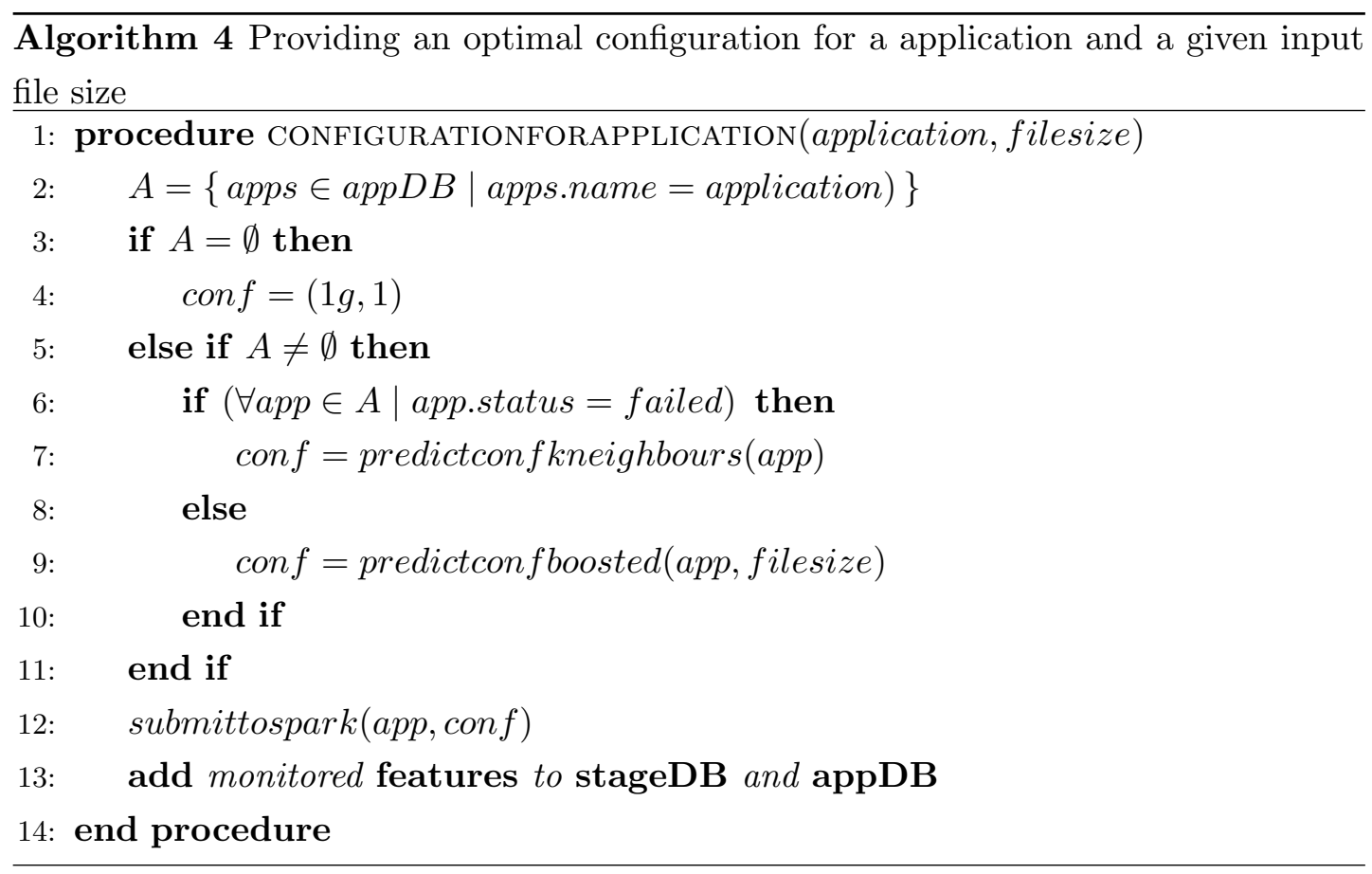

are Hadoop 2.6.0 and Spark 1.6.2. We configure the Hadoop cluster with six nodes working as datanodes, one of them as master node and the other five as nodemanagers. Each nodemanager has $21 \mathrm{~GB}$ of memory available for containers. We set the value of vcores to the same number as physical cores. We build a benchmark that is a combination of Spark-bench, Bigdatabench and some workloads that we implemented on our own. The latter ones were implemented to have a broader variety of stages and each one has different nature:

- RDDRelation: Here we use Spark SQL and the dataframes API. We read a file that has pairs of (key, value). First we count the number of keys using the RDD API and then using the Dataframe API with a select count $(*)$ type of query. After that, we do a select from query in a range of values and store the data in a Parquet data format 51 . This workflow consist of four stages in total.

- NGramsExample: We read a text file and we construct a dataframe where a row is a line in the text. Then we separate these lines in words and we calculate the NGrams=2 for each line. Finally these Ngrams are saved in a text file in HDFS.

\footnotetext{
${ }^{51}$ https://parquet.apache.org/
} 
This gives us a stage in total since it is all a matter of mapping the values and there is no shuffles involved.

- GroupByTest: Here we do not read any data from disk but we rather generate an RDD with pairs of (randomkey,values). The keys are generated inside a fixed range so there will be duplicate keys. The idea is to group by key and then perform a count of each key. The number of pairs, the number of partitions of the RDD and consequently the number of tasks can be changed through the input parameters of the application. This gives us two stages like in a typical GroupBy operation.

We run all the applications of this unified benchmark with three different file sizes as we want to check how the model reacts when executing the same application with different amounts of data. Some implementations, like the machine learning ones, expect a series of hyperparameters, which change the behaviour of the application. These are kept constant across executions and their effect is left out of the scope of this paper. We also try different values for spark. executor.memory and spark. executor.cores for each application and data size combinations. These executions generate a series of traces that we use to train and evaluate our model offline. Since the usefulness of our approach is that it can predict the performance of new unseen applications, we train the model by leaving some apps outside of the training set. Those apps will be later used as a test set. A description of the benchmark is depicted in Table 7.2 . The reason for choosing this benchmark, is to have a representative collection of batch processing applications that are affected differently by their parallelization, i.e. graph processing, text processing and machine learning. These three groups allow us to prove that applications with different resource consumption profiles have different optimal configurations. The files were generated synthetically. At the end we have 5583 stages to work with. As before we use the dynamic allocation feature of Spark and the DefaulResourceAllocator for YARN.

\subsubsection{Overhead of the system}

In this section, we want to evaluate the overhead of monitoring the system, training the model and calculating a prediction. The first concern is if Slim and GMonE introduce 


\begin{tabular}{|c|c|c|c|}
\hline Application & Dataset Split & File Size & Type of App \\
\hline ShortestPath & \multirow{6}{*}{ Test } & \multirow{2}{*}{$20 \mathrm{~GB}, 11 \mathrm{~GB}, 8 \mathrm{~GB}$} & \multirow{2}{*}{ Graph Processing } \\
\hline Connected Component & & & \\
\hline Logistic Regression & & \multirow{4}{*}{ 18GB, $10 \mathrm{~GB}, 5 \mathrm{~GB}$} & \multirow{3}{*}{ Machine Learning } \\
\hline Support Vector Machine & & & \\
\hline Spark PCA Example & & & \\
\hline Grep & & & Text Processing \\
\hline SVDPlusPlus & \multirow{12}{*}{ Train } & \multirow{4}{*}{ 20GB, $11 \mathrm{~GB}, 8 \mathrm{~GB}$} & \multirow{4}{*}{ Graph Processing } \\
\hline Page Rank & & & \\
\hline Triangle Count & & & \\
\hline Strongly Connected Component & & & \\
\hline Linear Regression & & \multirow{7}{*}{ 18GB,10GB,5GB } & \multirow{3}{*}{ Machine Learning } \\
\hline kMeans & & & \\
\hline Decision Tree & & & \\
\hline Tokenizer & & & \multirow{3}{*}{ Text Processing } \\
\hline Sort & & & \\
\hline WordCount & & & \\
\hline RDDRelation & & & SparkSQL \\
\hline GroupBy & & 100 tasks & Shuffle \\
\hline
\end{tabular}

Table 7.2: Different applications used. File size denotes the three different sizes used for that application. Group by doesn't read any file but creates all the data directly in memory with 100 partitions

some overhead when launching applications. We executed five batches of two applications running in succession: Grep and LinearRegression. We measured the time it took to execute these five batches with and without monitoring the applications. The results are depicted in Figure 7.11. As we can see, the overhead is negligible.

Also we want to evaluate how much time it takes to train the model and to calculate the optimal configuration for an application. We train the model with the traces we got from Grid'5000 in our local computer. The specifications for the CPU are 2,5 GHz Intel Core i7 of 4 cores and for the memory is 16GB of DDR3 RAM. The overhead of training the model depends on the number of data points, as shown in Figure 7.12. For the complete training set of 55139 the latency is 877 seconds. Note that this process is run on a laptop but it can be run on a node of the cluster with less load and more computation power. We must also consider that we trained the model with sklearn, 
Overhead of the monitoring system

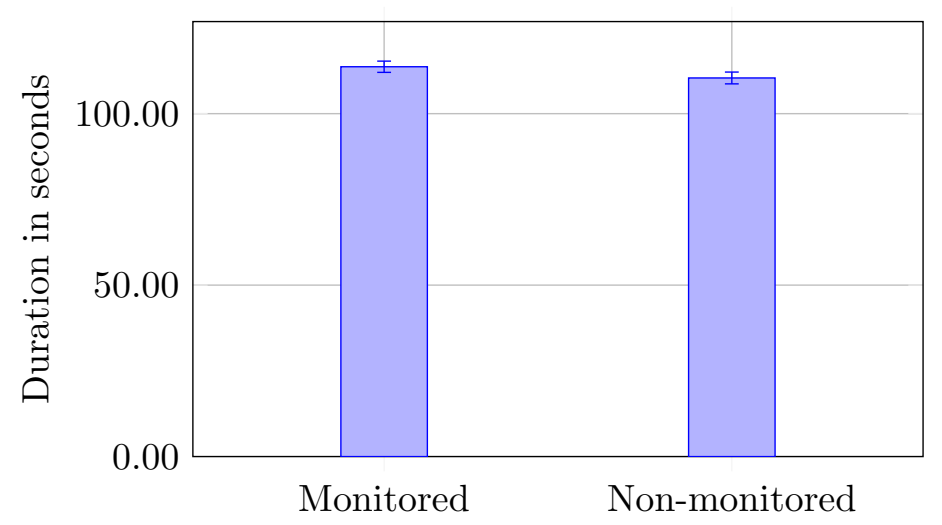

Figure 7.11: Overhead introduced by monitoring and storing the traces versus nonmonitoring. As we can see the latency is minimum

but there are distributed implementations of boosted regression trees in MLlib 52 for Spark that can speed up the process For the overhead of the predictions, we take all the data points in the test set for each application and predict their execution times. As we can see in Figure 7.13 , the overhead is negligible, with a maximum latency of 0.551 seconds for the LogisticRegression App that has 10206 points in the test set.

\subsubsection{Accuracy of predicted times}

Now we evaluate the accuracy of the predicted durations. As we mentioned earlier, we built the training and the test set by splitting the benchmark in two different groups. First, we start by evaluating the duration predictions for different stages. To do that, we take all the data points for a stage, we feed them to the model and we average the predictions for each configuration. Some of the results can be seen in Figure 7.14. For some stages, the predictions are really precise, like the stage 0 of Shortest Path with 8GB. Some others, like the Stage 1 of Support Vector Machine for 10GB, do not follow the exact same trend as the real duration but effectively detect the minimum. We show here some of the longest running stages across applications, since optimizing their performance will have a greater impact on the overall execution. Remember that our final objective is not to predict the exact run time but rather to know which configurations will affect negatively or positively the performance of an application.

\footnotetext{
${ }^{52}$ http://spark.apache.org/mllib/
} 


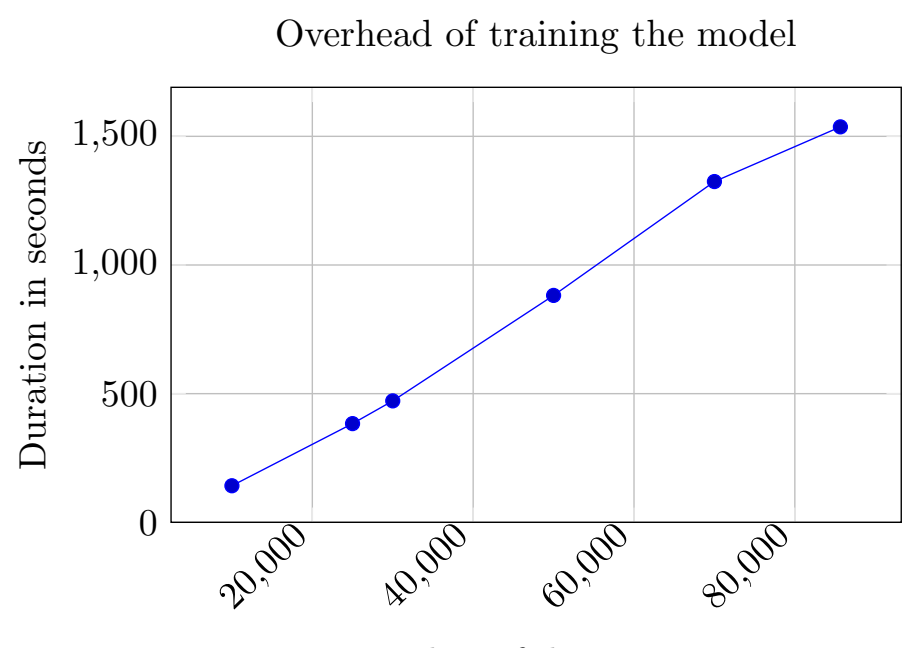

Number of data points

Figure 7.12: Duration of training the model depending on the number of data points. For the whole dataset of 85664 points the duration is 1536 seconds.

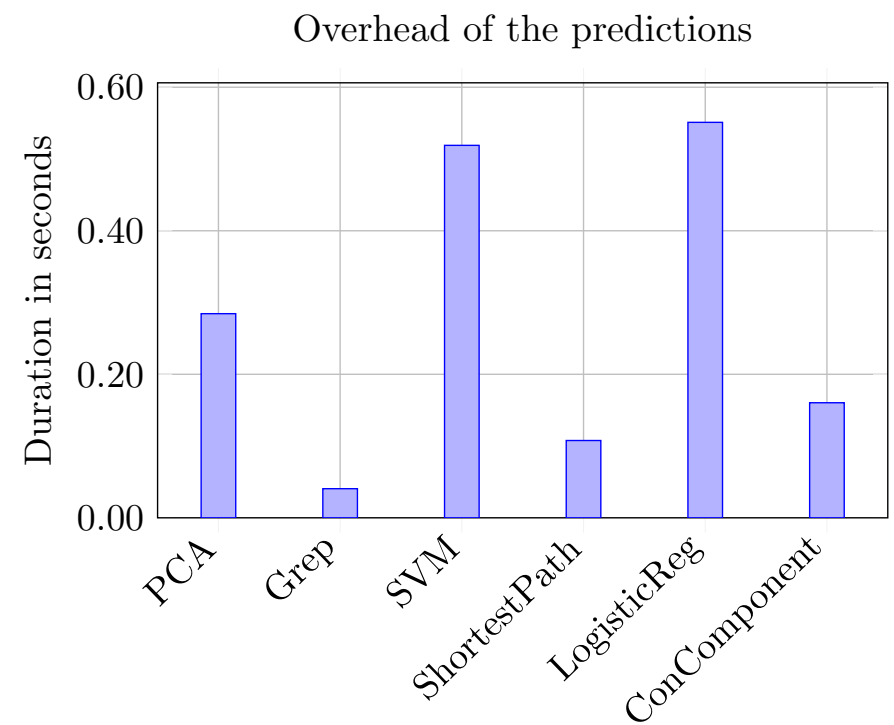

Figure 7.13: Latency in the predictions for different applications. The latency is always less than 1 second with a maximum of 0.519 for a Logistic Regression App 
Stage 0 of 8GB Shortest Path

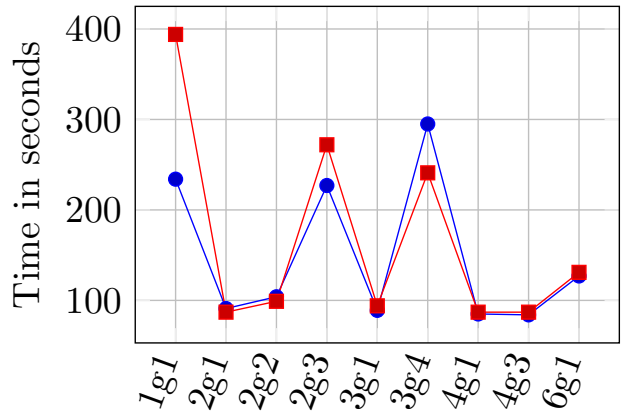

Stage 1 of 10GB SVM

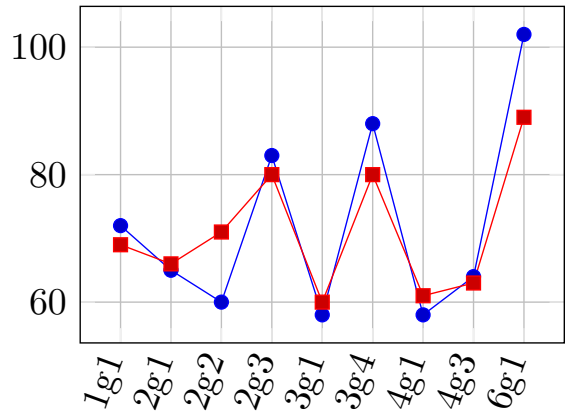

Stage 1 of 18GB Logistic Regression

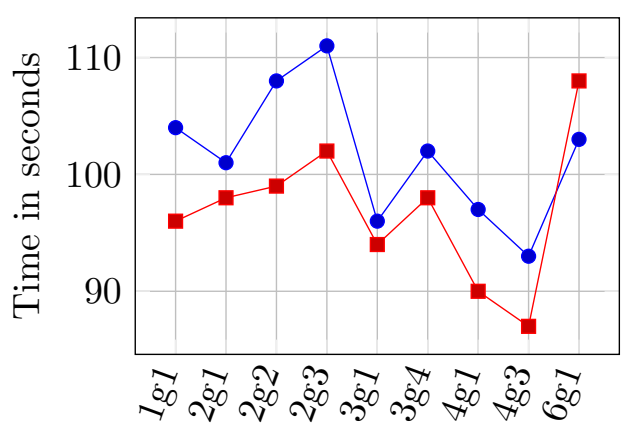

$\rightarrow \quad$ Real duration

- Predicted duration

Figure 7.14: Predictions for stages of different apps with the highest duration and so highest impact inside their applications. For Shortest Path the predictions are pretty accurate. For SVM and Logistic Regression the trend of the predicted values follows the real ones.

\subsubsection{Accuracy of parallelism recommendations for an application}

Now that we have seen the predictions for separate stages, we are going to evaluate the method for a complete application. The objective is to quantify the benefits of our recommendations compare to other three possible configurations: the default, best and worst one. We also want to evaluate how the predictions evolve with time, as we add more data points to our database of executions. To achieve that, we are going to assume a scenario where the user launches a set of applications recurrently with different file sizes. If the application has not been launched before, the recommendation will be the default one. After that first execution, the system will have monitored metrics for that application. We can start recommending configurations and the user will always choose to launch the workload with that configuration. In the scenario, for the non- 
Non-graph executions

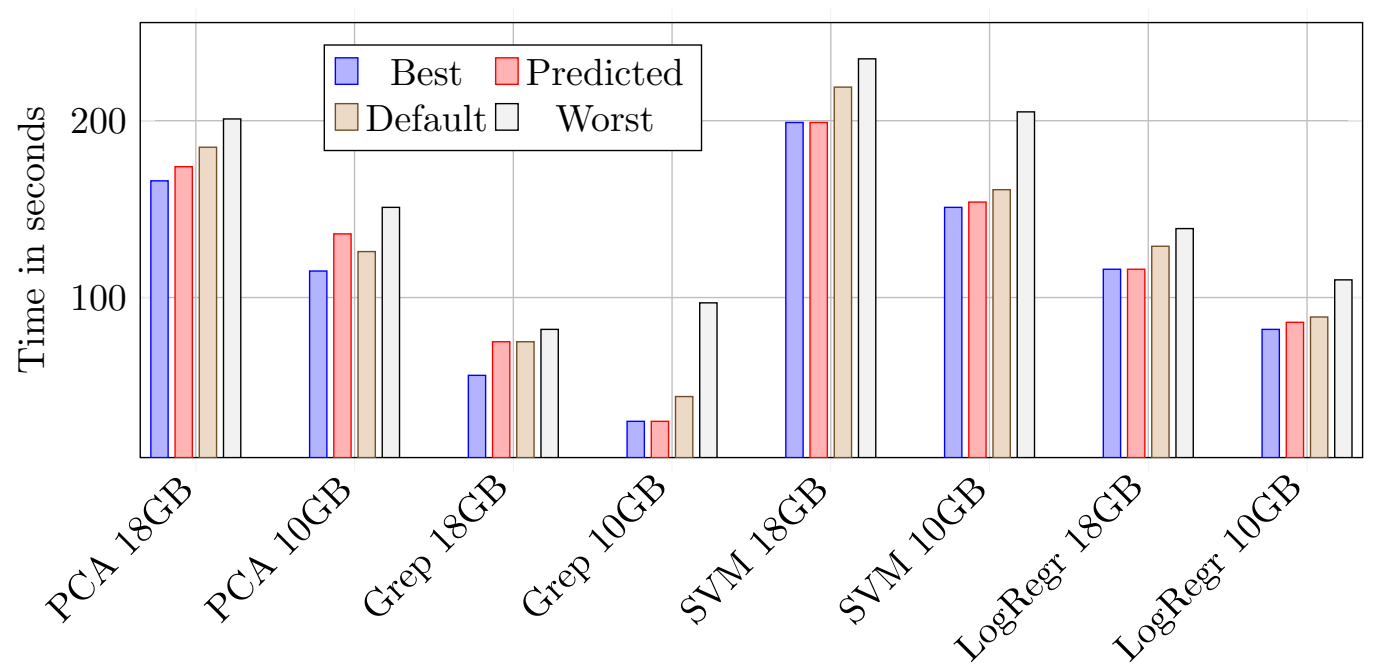

Figure 7.15: Executions for the non-graph processing applications of the test set.

graph applications in the test set, the user executes each one with 5GB, 10GB and 18GB consecutively. For the graph applications we do the same with their 20GB, 8GB and $11 \mathrm{~GB}$ sizes. In this case, we invert the order, since none of the $20 \mathrm{~GB}$ executions finished correctly because of out of memory errors and we use them to get the metrics instead. The results of these executions can be seen in Figures 7.15 and 7.16 . Note how we separate the figures for graphs and non-graph applications. The reason is that with one gigabyte for spark. executor.memory, the graph workloads always crashed, so we do not include this default execution. Best shows the lowest possible latency that we could get for that application. Predicted is the configuration proposed by our model. Default is the runtime for a $1 \mathrm{~g} / 1$ core configuration. Worst is the highest run time amongst the executions that finished successfully. We do not show here any application that did not complete successfully.

For some applications the recommendations achieve the maximum efficiency like Grep with 10GB, Logistic Regression with 18GB or SVM with 18GB. For example in Grep 10GB the maximum improvement is of $11 \%$. This means $11 \%$ of savings in energy and resource utilization, which may be significant when these applications are executed recurrently. These recommendations can also help the user to avoid really inefficient configurations, like the one in Grep 10GB. For graph executions, the benefits are more 


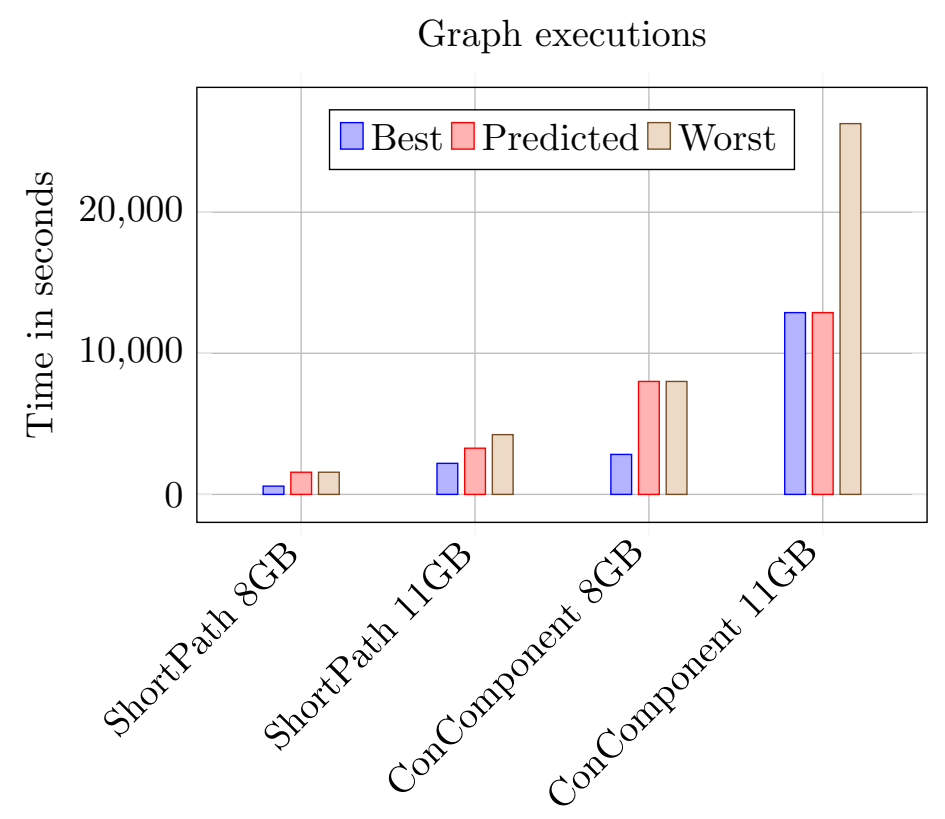

Figure 7.16: Executions for the graph processing applications of the test set.

obvious. Since the initial default execution always crashed, the first recommendation by the system was drawn out of the neighbours procedure explained early. The latency for this first recommendation is not optimal, but we have to keep in mind that this is a conservative approach to get a first complete run and a series of traces for the application. After getting this information and applying the boosted model, we can get an improvement up to $50 \%$ for the case of connected component with 11GB. We also want to prove that the model can converge to a better solution with every new execution it monitors. Note that we are not talking about retraining the model, but about using additional monitored executions to improve the accuracy. As an example, we keep executing the shortest path application first with 11GB and then with 8GB until the model converges to an optimal solution. The evolution can be seen in Figure 7.17

For the $11 \mathrm{~GB}$ execution, the first point is the duration for the neighbours approach. After that, the execution recommended with the boosted regression model fails, but the model goes back to an optimal prediction in the next iteration. In the $8 \mathrm{~GB}$ case, it goes down again from the conservative first recommendation to a nearly optimal one. This is an added value of the model. The system can become more intelligent with 


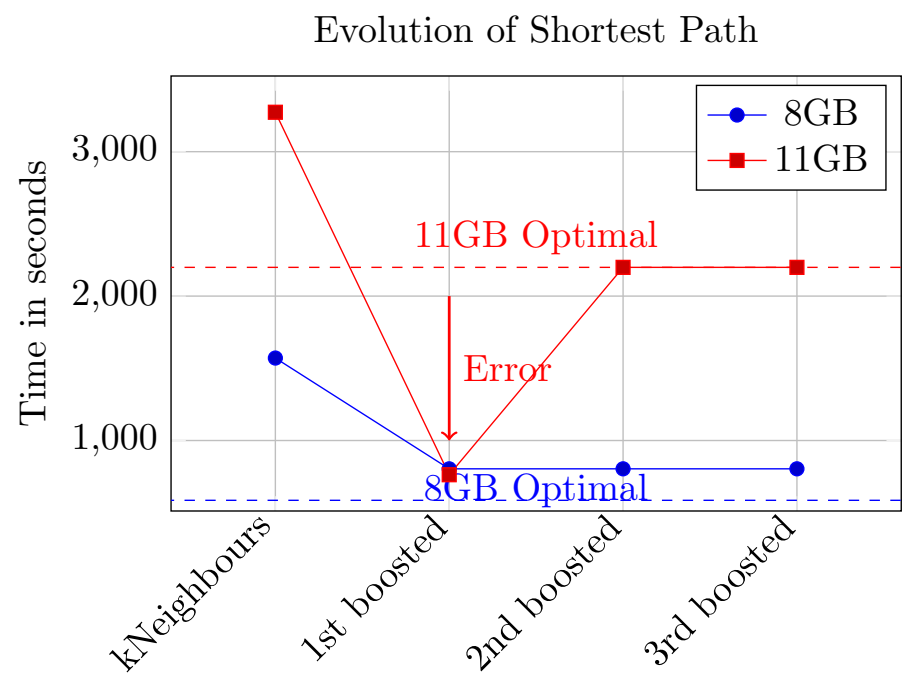

Figure 7.17: Evolution for the predictions of a recurrently executed shortest path application.

every new execution and will help the user to make better decisions about parallelism settings.

Finally, we include in Figure 7.18 the time that it takes to find an optimal configuration for each application. For the boosted decision trees approach, the average overhead is 0.3 seconds and it increases slightly for those applications that have more stages, like logistic regression. We consider this negligible, since a 0.3 seconds delay will not be noticeable by the user. The kNeighbours approach is faster, but it is only applied to those applications that crash with the default execution of $1 \mathrm{~g} / 1$ core.

\subsubsection{Interpretability of the model}

Another advantage of decision trees is that they are interpretable. That means that we can evaluate the impact of certain features on the outcome variable (duration in our case). One of the possible applications is to explain how the number of tasks per node affects a given workload. This information can be valuable when the user wants to manually choose a configuration. For example, in Figure 7.19 we see the partial dependence of three applications. Shortest Paths is a graph processing application and, as we saw earlier, it benefits from a low number of tasks per node. KMeans sweet spot seems to be around a default 10-12 tasks per node. For PCA, the points between 0 and 
Time to calculate recommended configuration

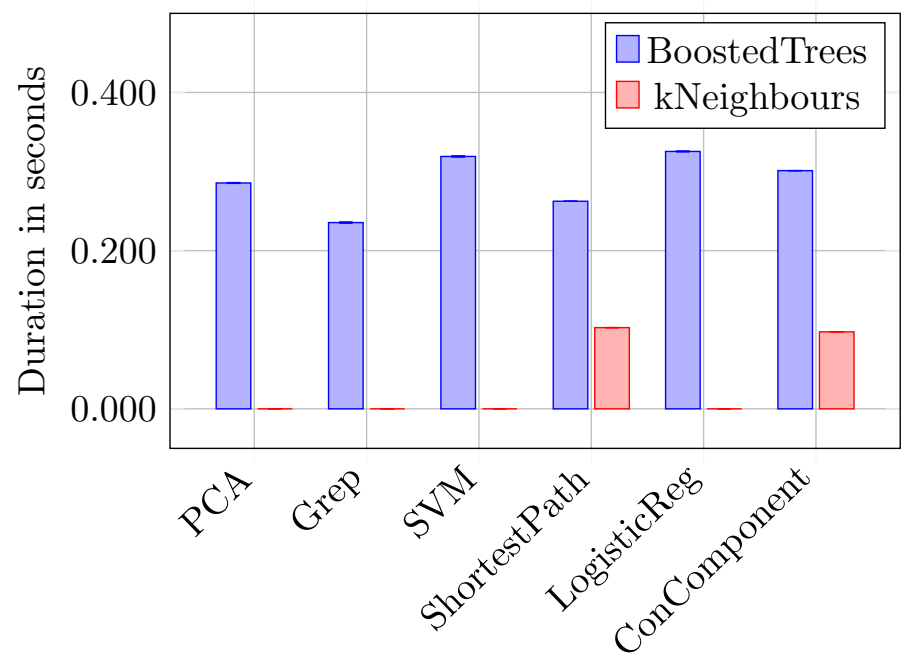

Figure 7.18: Time needed to calculate a recommended configuration with the boosted regression method and the kNeighbours method for each of the applications in our simulated scenario. Note that some of the applications do not use the kNeighbours method, since it is needed only when the application crashes with the default configuration of $1 \mathrm{~g}$ and 1 core

5 correspond to stages with only one or few tasks, something very common in machine learning implementations. Again everything from 8 to 15 is a good configuration while more than that, it is counterproductive. Also note how the partial dependence shows us the degree to which parallelism affects that workload. For example in Shortest Paths it ranges from 2000 to -4000 while in kMeans it goes from 100 to -100 . Indeed, as we saw in the experiments of the motivation section, the benefit of parallelism is more obvious on graph applications than in kMeans. This feature of a decision tree proves to be useful and can help the user to understand how different parameters affect their workloads.

\subsection{Conclusions: controlling and optimising big data work- loads}

Data analytics platforms are the last step inside the hierarchical data flow of IoT. In many cases, these platforms have to execute computational demanding workloads and 


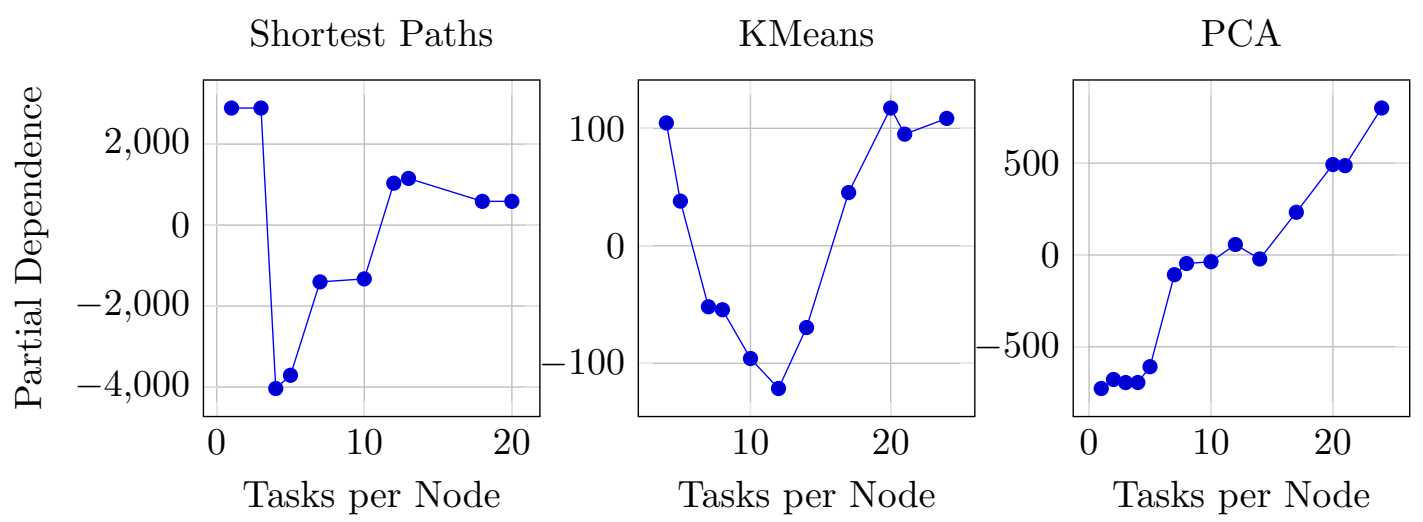

Figure 7.19: Partial dependence for different applications. Shortest Paths works better with a low number of tasks per node. kMeans best setting is 12 tasks per node. For PCA the points between 0 and 5 corresponds to stages with only one or few tasks, while with many tasks 7 is a good configuration.

optimising their performance can save both cost and time, which is specially important when these kinds of data analytics jobs are executed periodically. One of the most important decisions that is left to the user is setting the right level of parallelisation for the workload. That involves choosing a configuration that optimally assigns the CPU and memory resources needed by the tasks that are going to be distributed throughout the cluster. However studying the resource requirements of each application and deciding its right configuration can be cumbersome and prone to errors. In this chapter, we have shown that it is possible to accurately predict the execution time of a big data-based application with different file sizes and parallelism settings using machine learning models. Using these predictions we can decide which configuration parameters should we use to launch an application in an optimal way. These recommendations can be used as part of the decision support layer that we have detailed during this thesis, interacting with the user and informing of possible optimisations. 


\section{Part IV}

\section{Conclusions: Achievements and Perspectives}





\section{Chapter 8}

\section{Conclusions}

Over the last decade, we have seen the emergence of innovative applications that leverage the overwhelming amount of data generated by devices and users. Many of these so called Big Data applications run in distributed infrastructures, made up by many technologies and devices that share the same environment. Ensuring their performance becomes a problem even for the most experienced engineers, as the mixture of components creates a staggering complexity in which it is difficult to delve into. In addition, a new resurgence of decentralised infrastructures in the form of IoT and Fog computing, complicates the process even further, with more unstable devices and different virtualisation layer, to name some challenges.

There are many tools that provide observability into these distributed systems in the form of monitoring frameworks, which we have explored throughout this thesis. However, not many of them are able to extract knowledge from the information and metrics compiled. Human experts are the ones making decisions based on dashboards or other key performance indicators. Besides the inevitable risk of the decision making process, much information is left on the way, since the user will not be able to exhaustively analyse the huge amount of information generated in the monitoring process.

In this thesis we have explored several challenges related to the management of the new technologies linked with IoT. We described the complexities of monitoring new decentralised systems such as the Fog, and explained the design of a tool called FMonE, which is meant to provide observability into decentralised and heterogeneous systems. We continued by using different artificial intelligence techniques that are 
able to extract knowledge from the monitored information. We then take these AI models and implement prototypes that are meant to be part of a decision support framework, with which users can maintain and optimise decentralised systems like Fog computing. The framework followed a general workflow in which we monitored the relevant technologies, pre-processed and integrated the data, trained the AI models and finally used them to guide our decision-making process. Specifically, we tackled two problems in this context: root cause analysis for microservices and the optimisation of parallelism parameters for Big Data workloads. The different steps taken for each problem are shown in Table 8.1, in which we can observe the parallelism between the two approaches. We experimentally proved the accuracy of our models with a series of benchmarks and scenarios executed in the Grid'5000 testbed, which try to emulate the types of Big Data workloads that can be seen in industry. The results validate our previous hypothesis stating that artificial intelligence can be used to improve the performance and management of the different decentralised technologies used in IoT infrastructures.

\begin{tabular}{|c|c|c|c|c|}
\hline Problem & $\begin{array}{l}\text { Monitor } \\
\text { infrastruc- } \\
\text { ture }\end{array}$ & $\begin{array}{c}\text { Process } \\
\text { and explore } \\
\text { the data }\end{array}$ & $\begin{array}{l}\text { Train AI } \\
\text { models }\end{array}$ & $\begin{array}{l}\text { Making decisions based } \\
\text { on the model }\end{array}$ \\
\hline $\begin{array}{c}\text { RCA for } \\
\text { Microser- } \\
\text { vices } \\
\text { (Chapter 6. }\end{array}$ & $\begin{array}{c}\text { Sysdig }+ \\
\text { Prometheus }\end{array}$ & $\begin{array}{l}\text { Building } \\
\text { multi- } \\
\text { attributed } \\
\text { graphs }\end{array}$ & $\begin{array}{l}\text { Inexact } \\
\text { Graph } \\
\text { Matching }\end{array}$ & $\begin{array}{l}\text { Find nearest graph and its } \\
\text { associated root cause. Based } \\
\text { on the similarity score } \\
\text { (confidence) we can either } \\
\text { directly apply or suggest a } \\
\text { contingency plan. If } \\
\text { confidence is not high } \\
\text { enough, request manual } \\
\text { labelling from the user. }\end{array}$ \\
\hline $\begin{array}{l}\text { Optimising } \\
\text { parallelism } \\
\text { for Big } \\
\text { Data }\end{array}$ & $\begin{array}{c}\text { Slim } \\
\text { (Chapter } 7 \\
+ \text { GMonE }\end{array}$ & $\begin{array}{c}\text { Combining } \\
\text { parallelism } \\
\text { features + } \\
\text { application } \\
\text { metrics + } \\
\text { system } \\
\text { metrics }\end{array}$ & $\begin{array}{c}\text { Boosted } \\
\text { Decision } \\
\text { Trees }\end{array}$ & $\begin{array}{l}\text { Predict duration for different } \\
\text { parallelism parameters and } \\
\text { choose the most effective one }\end{array}$ \\
\hline
\end{tabular}

Table 8.1: The different steps used in our decision making workflow (Figure 4.1) and how they were used for the two technologies 
To conclude this thesis, we will list the different achievements and how they align with the objectives we established at the beginning. Finally, we discuss limitations of our work and future research lines that we have left unexplored.

\subsection{Achievements}

By integrating the following services into a common framework, users could benefit from a user decision support system oriented to the management of IoT-enabling technologies. In particular they can use the following features:

FMonE as a tool to provide observability for Fog environments. We first studied the different requirements and characteristics that a Fog monitoring tool should fulfill. Based on them, we developed a framework that is deployed using container technologies. It uses a monitoring pipeline abstraction, in which the metrics are extracted and sent/stored across different areas of the Fog hierarchical structure. We proved through our experiments and a qualitative analysis, that FMonE is able to meet the requirements we gathered and that containers are an enabling technology for Fog computing.

An accurate root cause analysis model for microservices. The microservices paradigm is one of the main technologies used in Fog and it has many synergies with decentralised infrastructures. However, it is also a complex technology in which troubleshooting a problem can become a challenge. By using graphs to model the interactions between containers, we are able to extract and save subgraphs that represent an anomaly in a given part of the architecture. These subgraphs are stored and labelled with its root cause in a library. Through the use of graph similarity techniques, we are able to compare any new anomalies that happen in the system with the graphs patterns in the library. The most similar patterns are then presented to the user as possible root causes. The accuracy of this model was proved through a series of representative microservice architectures. We further explored this area by collaborating with other authors to include information coming from logs into these graphs, enabling the transfer of the RCA knowledge acquired from one architecture to another. 


\section{A model to optimise the task parallelism for in-memory Big Data analytics}

platforms. We proposed a model that is able to save both costs and time when running analytical jobs by optimising the way their tasks are sized in terms of memory and cpu. By using machine learning techniques, we are able to learn from the resource usage of different Apache Spark applications, which parallelism parameters work best for each workload. The advantage of using machine learning is that the model can adapt itself to any new infrastructure or application executed.

\subsection{Tools and artifacts for reproducibility}

All of our experiments were performed in Grid'5000. This testbed provides several tools to create reproducible experiments (121). We scripted most of our experiments through exec(53, a Python library that facilitates the management of UNIX processes in a distributed infrastructure. In this way, it is much easier to prototype our experiments. It also allows other researchers to redeploy the same environments we used. The three main execo-based projects that can be used for reproducible research are:

- execo-utilities-g5l 54 An easy way to script reproducible experiments on the g5k testbed. It uses a series of Python classes to deploy experiments in an easy, scripted way that facilitate the reservation of nodes in G5K, the deployment, the execution of the experiment and the preservation of the results.

- execo-g5k-benchmark $\{55$ It can be used together with execo-utilities-g5k to facilitate the execution of some of the benchmarks we detailed in Section 2.2.3.3.

- microservices-rca 56 It provides methods to interact with DCOS and launch the microservice architectures we have used during this thesis. It also allows to inject anomalies in the different machines that will make up the infrastructure

We have also created some artifacts in the process that are needed by several of the previous projects:

\footnotetext{
${ }^{53}$ http://execo.gforge.inria.fr/doc/latest-stable/

${ }^{54}$ https://github.com/Brandonage/execo-utilities-g5k

${ }^{55}$ https://github.com/Brandonage/execo-g5k-benchmarks

${ }^{56}$ https://github.com/Brandonage/microservices-rca
} 
- Docker YCSB imag£57. A Docker Image that can be used to generate any of the YCSB workloads in a lightweight and elastic way.

- Docker SparkBench imag£58, A Docker Image that can be used to launch any of the SparkBench workloads

- DCOS VM imag $£^{59}$; The QCOW ${ }^{60}$ file needed by vagrant-g5k to deploy the VM's in which we run all of our DCOS experiments for microservice architectures.

These tools can facilitate the deployment of similar experiments, helping other researchers to make advancements in the field.

\subsection{Future Work}

During the work of this thesis we have focused on some research lines, but left others behind that can be undertaken in the future. In addition, we have also identified new problems and challenges that we address in this section.

Root cause analysis for numerous IoT devices. We have used containers and microservice architectures to validate our graph-based RCA method. Although microservices are one of the main pillars of a Fog architecture, we also want to test the advantages of our method when detecting failures at the physical layer. This could include problems like detecting sensors that are not sending valid measurements, problems in the interconnection between devices or overloaded gateways. Our graph approach can perfectly adapt to the heterogeneity of IoT thanks to its modularity and its ability to accommodate to a target domain. For instance, the different communication protocols can be embedded as attributes in the edges, or the different sensor modules and their manufacturers included in a user-built taxonomy.

Improving graph similarity calculation time. In the context of our RCA approach, we have calculated the similarity between graphs up to 30 nodes. However, graphs in big production systems could be much larger than that. This is

\footnotetext{
${ }^{57}$ https://hub.docker.com/r/alvarobrandon/ycsb/

${ }^{58}$ https://hub.docker.com/r/alvarobrandon/spark-bench/

${ }^{59}$ https://zenodo.org/record/1325049

${ }^{60}$ https://www.linux-kvm.org/page/Qcow2
} 
specially relevant if we want to test it in an IoT environment where there are numerous elements. The graph similarity calculation between two large graphs could turn out to be expensive in terms of computation time and not suitable for real-time diagnostics of the system. Graph indexing techniques based on the similarity function can be one of the solutions (17). Another option could be vectorising the graph representation through graph embedding techniques (45), as it is much easier to compare two elements as a vector than by their graph structure.

Including time dimension in the graph representation of the system. Many anomalies can be identified by the way they evolve in the system. For example, a memory leak in a JVM will start with a slow increase of heap memory used, followed by an increase of the cpu activity due to the garbage collection and then the final crash. This evolution of the system during a time window can be represented as a sequence of snapshots of the system, instead of single graphs. Its usefulness could be proved in a serverless environment, where containers come and go depending on specific events.

Task contention and scheduling depending on the type of workload. We have already mentioned how difficult is to fully control and optimise the usage of a distributed infrastructure. One of the reasons is the difficulty of intelligently placing different tasks throughout the machines in such a way that the full capacity of the hardware can be reached (92). Containerisation makes it very easy to migrate applications from one host to another, enabling the consolidation of workloads into a few hosts. However, task contention should be taken into account, as placing several workloads that use the same kind of resource would be counterproductive (i.e. placing I/O intensive workloads in the same host). New research into intelligent scheduling methods would allow to collocate tasks more efficiently cutting costs in infrastructure and energy.

New visualisation and representation techniques for IoT deployments. Many of the models developed during this thesis can be implemented as part of a decision support systems. Another desirable feature of a DSS is delivering visualizations (e.g. dashboards) that facilitate to the users the comprehension of their applications and infrastructure. However, an IoT deployment could be very large, with 
thousands of devices to monitor and each one with many heterogeneous characteristics. So far, commercial tools provide visualisations for single site or multi-site datacenters, but not for IoT infrastructures, which we believe can be a research line itself. 


\section{Bibliography}

[1] Dynamic allocation in spark. http://spark.apache.org/docs/latest/ job-scheduling.html/. 123

[2] Electrical consumption on grid'5000 lyon site. https://intranet.grid5000. fr/supervision/lyon/wattmetre/. 124

[3] Managing cpu resources. https://hadoop.apache.org/docs/r2.7.0/ hadoop-yarn/hadoop-yarn-site/CapacityScheduler.html. 124

[4] Abderrahim, M., Ouzzif, M., Guillouard, K., Francois, J., and Lèbre, A. A holistic monitoring service for fog/edge infrastructures: a foresight study. In The IEEE 5th International Conference on Future Internet of Things and Cloud (FiCloud 2017) (2017), pp. 337-344. 40

[5] Abidin, S. A. H. Z., And Ibrahim, S. N. Web-based monitoring of an automated fertigation system: An iot application. In Communications (MICC), 2015 IEEE 12th Malaysia International Conference on (2015), IEEE, pp. 1-5. 65

[6] Akoglu, L., Tong, H., And Koutra, D. Graph based anomaly detection and description: a survey. Data Mining and Knowledge Discovery 29, 3 (2015), 626-688. 36

[7] Akoglu, L., Tong, H., And Koutra, D. Graph based anomaly detection and description: a survey. Data mining and knowledge discovery 29, 3 (2015), 626-688. 88

[8] Al-Dhuraibi, Y., Paraiso, F., Duarallah, N., and Merle, P. Elasticity in cloud computing: state of the art and research challenges. IEEE Transactions on Services Computing 11, 2 (2018), 430-447. 21 
[9] Al-Fuqaha, A., Guizani, M., Mohammadi, M., Aledhari, M., and AYYASH, M. Internet of things: A survey on enabling technologies, protocols, and applications. IEEE Communications Surveys $\mathcal{E}$ Tutorials 17, 4 (2015), 23472376. 15]

[10] Alhosban, A., Hashmi, K., Malik, Z., and Medjahed, B. Self-healing framework for cloud-based services. In Computer Systems and Applications (AICCSA), 2013 ACS International Conference on (2013), IEEE, pp. 1-7. 34

[11] Alsheikh, M. A., Niyato, D., Lin, S., Tan, H.-P., and Han, Z. Mobile big data analytics using deep learning and apache spark. IEEE network 30, 3 (2016), 22-29. 25

[12] Arasu, A., Cherniack, M., Galvez, E., Maier, D., Maskey, A. S., Ryvkina, E., Stonebraker, M., And Tibbetts, R. Linear road: A stream data management benchmark. In Proceedings of the Thirtieth International Conference on Very Large Data Bases - Volume 30 (2004), VLDB '04, VLDB Endowment, pp. 480-491. 26

[13] Arlitt, M., Marwah, M., Bellala, G., Shah, A., Healey, J., and VanDIVER, B. Iotabench: An internet of things analytics benchmark. In Proceedings of the 6th ACM/SPEC International Conference on Performance Engineering (New York, NY, USA, 2015), ICPE '15, ACM, pp. 133-144. 28

[14] Balouek, D., Carpen Amarie, A., Charrier, G., Desprez, F., Jeannot, E., Jeanvoine, E., Lèbre, A., Margery, D., Niclausse, N., Nussbaum, L., Richard, O., Pérez, C., Quesnel, F., Rohr, C., and Sarzyniec, L. Adding virtualization capabilities to the Grid'5000 testbed. In Cloud Computing and Services Science, I. Ivanov, M. Sinderen, F. Leymann, and T. Shan, Eds., vol. 367 of Communications in Computer and Information Science. Springer International Publishing, 2013, pp. 3-20. 69, 99, 124

[15] Bellavista, P., And Zanni, A. Feasibility of fog computing deployment based on docker containerization over raspberrypi. In Proceedings of the 18th International Conference on Distributed Computing and Networking (2017), ACM, p. 16. 
[16] Berral, J. L., Poggi, N., Carrera, D., Call, A., Reinauer, R., And GreEn, D. Aloja-ml: A framework for automating characterization and knowledge discovery in hadoop deployments. In Proceedings of the 21th ACM SIGKDD International Conference on Knowledge Discovery and Data Mining (2015), ACM, pp. 1701-1710. 37

[17] Berretti, S., Del Bimbo, A., And Vicario, E. Efficient matching and indexing of graph models in content-based retrieval. IEEE Transactions on Pattern Analysis and Machine Intelligence 23, 10 (2001), 1089-1105. 111, 162

[18] Bonomi, F., Milito, R., Natarajan, P., and Zhu, J. Fog computing: A platform for internet of things and analytics. In Big Data and Internet of Things: A Roadmap for Smart Environments. Springer, 2014, pp. 169-186. 56

[19] Bormann, C., Castellani, A. P., And Shelby, Z. Coap: An application protocol for billions of tiny internet nodes. IEEE Internet Computing 16, 2 (2012), 62-67. 15

[20] Botta, A., De Donato, W., Persico, V., And Pescapé, A. Integration of cloud computing and internet of things: a survey. Future Generation Computer Systems 56 (2016), 684-700. 14

[21] Brandon, A., Marc, S., Huelamo, A., Solans, D., Perez, M. S., And Muntes, V. Graph-based root cause analysis for microservice architectures. In press (2018). 84

[22] Brandón, Á., Pérez, M. S., Montes, J., And Sanchez, A. Fmone: A flexible monitoring solution at the edge. Wireless Communications and Mobile Computing 2018 (2018). 7, 55

[23] Bruno, N., Agarwal, S., Kandula, S., Shi, B., Wu, M.-C., And Zhou, J. Recurring job optimization in scope. Proceedings of the 2012 international conference on Management of Data - SIGMOD '12 (2012), 805. 142

[24] Callaway, E., Gorday, P., Hester, L., Gutierrez, J. A., Naeve, M., Heile, B., AND BAHL, V. Home networking with ieee 802.15. 4: a developing standard for low-rate wireless personal area networks. IEEE Communications magazine 40, 8 (2002), 70-77. 15 
[25] Cao, Y., Chen, S., Hou, P., and Brown, D. Fast: A fog computing assisted distributed analytics system to monitor fall for stroke mitigation. In Networking, Architecture and Storage (NAS), 2015 IEEE International Conference on (2015), IEEE, pp. 2-11. 40

[26] Carbone, P., Katsifodimos, A., Ewen, S., Markl, V., Haridi, S., And Tzoumas, K. Apache flink: Stream and batch processing in a single engine. Bulletin of the IEEE Computer Society Technical Committee on Data Engineering 36,4 (2015). 25, 29

[27] Cardellini, V., Colajanni, M., and Yu, P. S. Dynamic load balancing on web-server systems. IEEE Internet computing 3, 3 (1999), 28-39. 99

[28] Chandola, V., Banerjee, A., and Kumar, V. Anomaly detection: A survey. ACM computing surveys (CSUR) 41, 3 (2009), 15. 88

[29] Charlotte, D., Sabrine, F., and Dupont, C. Waziup: An internet of things and big data platform to boost innovation in rural africa, 2016. 26

[30] Chatzigiannakis, I., Hasemann, H., Karnstedt, M., Kleine, O., Kroller, A., Leggieri, M., Pfisterer, D., Romer, K., And Truong, C. True self-configuration for the iot. In Internet of Things (IOT), 2012 3rd International Conference on the (2012), IEEE, pp. 9-15. 36

[31] Coetzee, L., and Eksteen, J. The internet of things-promise for the future? an introduction. In IST-Africa Conference Proceedings, 2011 (2011), IEEE, pp. 1-9. 17

[32] Cooper, B. F., Silberstein, A., Tam, E., Ramakrishnan, R., and Sears, R. Benchmarking cloud serving systems with ycsb. In Proceedings of the 1st ACM Symposium on Cloud Computing (New York, NY, USA, 2010), SoCC '10, ACM, pp. 143-154. 28

[33] Dai, Y., Xiang, Y., And Zhang, G. Self-healing and hybrid diagnosis in cloud computing. In IEEE International Conference on Cloud Computing (2009), Springer, pp. 45-56. 34 
[34] Dastjerdi, A. V., And Buyya, R. Fog computing: Helping the internet of things realize its potential. Computer 49, 8 (2016), 112-116. 57

[35] Datadog - real-time performance monitoring, Sep. 2017. https://www. datadoghq. com. 42

[36] de Deugd, S., Carroll, R., Kelly, K., Millett, B., And Ricker, J. Soda: Service oriented device architecture. IEEE Pervasive Computing 5, 3 (2006), 9496. 17

[37] Dean, J., and Ghemawat, S. Mapreduce: simplified data processing on large clusters. Communications of the ACM 51, 1 (2008), 107-113. 25

[38] Demetgul, M. Fault diagnosis on production systems with support vector machine and decision trees algorithms. The International Journal of Advanced Manufacturing Technology 67, 9-12 (2013), 2183-2194. 35, 110

[39] Doran, D., Schulz, S., And Besold, T. R. What does explainable ai really mean? a new conceptualization of perspectives. arXiv preprint arXiv:1710.00794 (2017). 50

[40] Dynatrace, Sep. 2017. https://www.dynatrace.com/. 42

[41] Elith, J., Leathwick, J. R., And Hastie, T. A working guide to boosted regression trees. Journal of Animal Ecology 77, 4 (2008), 802-813. 110

[42] Estrada, R., And Ruiz, I. Big data smack a guide to apache spark, mesos, akka, cassandra, and kafka (2016). Tech. rep., ISBN 978-1-4842-2175-4, 2016. 100

[43] Falkenthal, M., Breitenbücher, U., Képes, K., Leymann, F., Zimmermann, M., Christ, M., Neuffer, J., Braun, N., and Kempa-Liehr, A. W. Opentosca for the 4th industrial revolution: Automating the provisioning of analytics tools based on apache flink. In Proceedings of the 6th International Conference on the Internet of Things (2016), ACM, pp. 179-180. 26

[44] Farahani, S. ZigBee wireless networks and transceivers. Newnes, 2011. 15 
[45] Foggia, P., Percannella, G., And Vento, M. Graph matching and learning in pattern recognition in the last 10 years. International Journal of Pattern Recognition and Artificial Intelligence 28, 01 (2014), 1450001. 162

[46] Foster, I., Kesselman, C., And Tuecke, S. The anatomy of the grid: Enabling scalable virtual organizations. The International Journal of High Performance Computing Applications 15, 3 (2001), 200-222. 41

[47] George, L. HBase: the definitive guide: random access to your planet-size data. " O'Reilly Media, Inc.", 2011. 28

[48] Gershenfeld, N., Krikorian, R., and Cohen, D. The internet of things. Scientific American 291, 4 (2004), 76-81. 14

[49] Ghazal, A., Rabl, T., Hu, M., Raab, F., Poess, M., Crolotte, A., And JaCobsen, H.-A. Bigbench: Towards an industry standard benchmark for big data analytics. In Proceedings of the 2013 ACM SIGMOD International Conference on Management of Data (New York, NY, USA, 2013), SIGMOD '13, ACM, pp. 1197-1208. 27

[50] Ghodsi, A., Zaharia, M., Hindman, B., Konwinski, A., Shenker, S., And StoicA, I. Dominant resource fairness: Fair allocation of multiple resource types. In NSDI (2011), vol. 11, pp. 24-24. 122

[51] Ghosh, D., Sharman, R., Rao, H. R., and Upadhyaya, S. Self-healing systems - survey and synthesis. Decision support systems 42, 4 (2007), 21642185. 33

[52] Greenberg, A., Hamilton, J., Maltz, D. A., and Patel, P. The cost of a cloud: research problems in data center networks. ACM SIGCOMM computer communication review 39, 1 (2008), 68-73. 74

[53] Grossmann, M., Eiermann, A., and Renner, M. Hypriot cluster lab: an arm-powered cloud solution utilizing docker. Tech. rep., Hypriot, 2016. 80

[54] Grossmann, M., And Klug, C. Monitoring container services at the network edge. In Teletraffic Congress (ITC 29), 2017 29th International (2017), vol. 1, IEEE, pp. 130-133. 40 
[55] Gubbi, J., Buyya, R., Marusic, S., and Palaniswami, M. Internet of things (iot): A vision, architectural elements, and future directions. Future generation computer systems 29, 7 (2013), 1645-1660. 13

[56] Guth, J., Breitenbücher, U., Falkenthal, M., Leymann, F., and ReINFURT, L. Comparison of iot platform architectures: A field study based on a reference architecture. In Cloudification of the Internet of Things (CIoT) (2016), IEEE, pp. 1-6. 17

[57] Haller, S., Karnouskos, S., and Schroth, C. The internet of things in an enterprise context. In Future Internet Symposium (2008), Springer, pp. 14-28. 29

[58] Heorhiadi, V., Rajagopalan, S., Jamjoom, H., Reiter, M. K., And SEKAR, V. Gremlin: systematic resilience testing of microservices. In Distributed Computing Systems (ICDCS), 2016 IEEE 36th International Conference on (2016), IEEE, pp. 57-66. 85

[59] Hernández, Á. B., Perez, M. S., Gupta, S., And Muntés-Mulero, V. Using machine learning to optimize parallelism in big data applications. Future Generation Computer Systems 86 (2018), 1076-1092. 8, 121

[60] Herodotou, H., And Babu, S. Profiling, what-if analysis, and cost-based optimization of mapreduce programs. Proceedings of the VLDB Endowment 4 , 11 (2011), 1111-1122. 37

[61] Herodotou, H., Lim, H., Luo, G., Borisov, N., Dong, L., Cetin, F. B., AND BABU, S. Starfish: A self-tuning system for big data analytics. In CIDR (2011), vol. 11, pp. 261-272. 37

[62] Hindman, B., Konwinski, A., Zaharia, M., Ghodsi, A., Joseph, A. D., Katz, R. H., Shenker, S., And Stoica, I. Mesos: A platform for fine-grained resource sharing in the data center. In NSDI (2011), vol. 11, pp. 22-22. 62

[63] Holzinger, A. Interactive machine learning for health informatics: when do we need the human-in-the-loop? Brain Informatics 3, 2 (2016), 119-131. 51 
[64] Hong, K., Lillethun, D., Ramachandran, U., Ottenwälder, B., And Koldehofe, B. Mobile fog: A programming model for large-scale applications on the internet of things. In Proceedings of the second ACM SIGCOMM workshop on Mobile cloud computing (2013), ACM, pp. 15-20. 19

[65] Horn, P. Autonomic Computing: IBM's Perspective on the State of Information Technology. Tech. rep., 2001. 32

[66] Huang, S., Huang, J., Dai, J., Xie, T., And Huang, B. The hibench benchmark suite: Characterization of the mapreduce-based data analysis. In New Frontiers in Information and Software as Services: Service and Application Design Challenges in the Cloud (Berlin, Heidelberg, 2011), D. Agrawal, K. S. Candan, and W.-S. Li, Eds., Springer Berlin Heidelberg, pp. 209-228. 28

[67] Huebscher, M. C., And McCann, J. A. A survey of autonomic computing - degrees, models, and applications. ACM Computing Surveys (CSUR) 40, 3 (2008), 7. xv, 32, 33

[68] Hung, C.-C., Golubchik, L., And Yu, M. Scheduling jobs across geodistributed datacenters. In Proceedings of the Sixth ACM Symposium on Cloud Computing (New York, NY, USA, 2015), SoCC '15, ACM, pp. 111-124. 25

[69] Hunt, P., Konar, M., Junqueira, F. P., And Reed, B. Zookeeper: Waitfree coordination for internet-scale systems. In USENIX annual technical conference (2010), vol. 8, Boston, MA, USA, p. 9. 63, 77

[70] Hwang, K., And Chen, M. Big-data analytics for cloud, IoT and cognitive computing. John Wiley \& Sons, 2017. 30

[71] Ismail, B. I., Goortani, E. M., Ab Karim, M. B., Tat, W. M., Setapa, S., Luke, J. Y., And Hoe, O. H. Evaluation of docker as edge computing platform. In Open Systems (ICOS), 2015 IEEE Confernece on (2015), IEEE, pp. 130-135. 22

[72] Jadeja, Y., And Modi, K. Cloud computing-concepts, architecture and challenges. In Computing, Electronics and Electrical Technologies (ICCEET), 2012 International Conference on (2012), IEEE, pp. 877-880. 42 
[73] Jamjoom, S. R. H. App-bisect: Autonomous healing for microservice-based apps. 35

[74] Ji, Z., Ganchev, I., O’Droma, M., Zhao, L., and Zhang, X. A cloud-based car parking middleware for iot-based smart cities: Design and implementation. Sensors 14, 12 (2014), 22372-22393. 26

[75] Joshi, K. R., Hiltunen, M. A., Sanders, W. H., And Schlichting, R. D. Automatic model-driven recovery in distributed systems. In Reliable Distributed Systems, 2005. SRDS 2005. 24th IEEE Symposium on (2005), IEEE, pp. 25-36. 35

[76] Joy, A. M. Performance comparison between linux containers and virtual machines. In Computer Engineering and Applications (ICACEA), 2015 International Conference on Advances in (2015), IEEE, pp. 342-346. 21

[77] Kephart, J. O., And Chess, D. M. The vision of autonomic computing. Computer 36, 1 (2003), 41-50. 32

[78] Kirsch, H., And Kroschel, K. Applying bayesian networks to fault diagnosis. In Control Applications, 1994., Proceedings of the Third IEEE Conference on (Aug 1994), pp. 895-900 vol.2. 35

[79] Kohavi, R., ET AL. A study of cross-validation and bootstrap for accuracy estimation and model selection. In Ijcai (1995), vol. 14, pp. 1137-1145. 137

[80] Koller, D., And Friedman, N. Probabilistic graphical models: principles and techniques. MIT press, 2009. 35

[81] Kosmatos, E. A., Tselikas, N. D., And Boucouvalas, A. C. Integrating rfids and smart objects into a unifiedinternet of things architecture. Advances in Internet of Things 1, 01 (2011), 5. $\mathrm{xv}, 14$

[82] Kumar, S., Padakandla, S., Parihar, P., Gopinath, K., Bhatnagar, S., ET AL. Performance tuning of hadoop mapreduce: A noisy gradient approach. arXiv preprint arXiv:1611.10052 (2016). 37 
[83] Lakshman, A., And Malik, P. Cassandra: A decentralized structured storage system. SIGOPS Oper. Syst. Rev. 44, 2 (Apr. 2010), 35-40. 28, 117

[84] Lama, P., And Zhou, X. Aroma: Automated resource allocation and configuration of mapreduce environment in the cloud. In Proceedings of the 9th international conference on Autonomic computing (2012), ACM, pp. 63-72. 37

[85] Lea, R., and Blackstock, M. City hub: A cloud-based iot platform for smart cities. In Cloud Computing Technology and Science (CloudCom), 2014 IEEE 6th International Conference on (2014), IEEE, pp. 799-804. 17

[86] Leung, A., Spyker, A., And Bozarth, T. Titus: introducing containers to the netflix cloud. Communications of the ACM 61, 2 (2018), 38-45. 83

[87] Li, M., Tan, J., Wang, Y., Zhang, L., And Salapura, V. Sparkbench: A comprehensive benchmarking suite for in memory data analytic platform spark. In Proceedings of the 12th ACM International Conference on Computing Frontiers (New York, NY, USA, 2015), CF '15, ACM, pp. 53:1-53:8. 27

[88] Liu, H., And WeE, S. Web server farm in the cloud: Performance evaluation and dynamic architecture. In IEEE International Conference on Cloud Computing (2009), Springer, pp. 369-380. 102

[89] Lu, J., Behbood, V., Hao, P., Zuo, H., Xue, S., and Zhang, G. Transfer learning using computational intelligence: A survey. Knowledge-Based Systems 80 (2015), 14 - 23. 25th anniversary of Knowledge-Based Systems. 112

[90] Luan, T. H., Gao, L., Li, Z., Xiang, Y., Wei, G., and Sun, L. Fog computing: Focusing on mobile users at the edge. arXiv preprint arXiv:1502.01815 (2015). xv, 19

[91] Marín-Tordera, E., Masip-Bruin, X., García-Almiñana, J., Jukan, A., Ren, G.-J., AND Zhu, J. Do we all really know what a fog node is? current trends towards an open definition. Computer Communications 109 (2017), 117130. 18 
[92] Mars, J., Tang, L., Hundt, R., Skadron, K., and Soffa, M. L. Bubbleup: Increasing utilization in modern warehouse scale computers via sensible colocations. In Proceedings of the 44th annual IEEE/ACM International Symposium on Microarchitecture (2011), ACM, pp. 248-259. 162

[93] Marston, S., Li, Z., Bandyopadhyay, S., Zhang, J., and Ghalsasi, A. Cloud computing - the business perspective. Decision support systems 51, 1 (2011), 176-189. 56

[94] Marvasti, M. A., Poghosyan, A. V., Harutyunyan, A. N., And GrigORYAN, N. M. An anomaly event correlation engine: Identifying root causes, bottlenecks, and black swans in it environments. VMware Technical Journal 2, 1 (2013), 35-45. 36

[95] Massie, M. L., Chun, B. N., And Culler, D. E. The ganglia distributed monitoring system: design, implementation, and experience. Parallel Computing 30, 7 (2004), 817-840. 41

[96] Matri, P., Alforov, Y., Brandon, A., Kuhn, M., Carns, P., and LudWIG, T. Could blobs fuel storage-based convergence between hpc and big data? In Cluster Computing (CLUSTER), 2017 IEEE International Conference on (2017), IEEE, pp. 81-86. 7, 24

[97] Matri, P., Alforov, Y., Álvaro Brandon, Pérez, M. S., Costan, A., Antoniu, G., Kuhn, M., Carns, P., and Ludwig, T. Mission possible: Unify hpc and big data stacks towards application-defined blobs at the storage layer. Future Generation Computer Systems (2018). 7, 24

[98] Mdhaffar, A., Halima, R. B., Jmaiel, M., and Freisleben, B. Reactive performance monitoring of cloud computing environments. Cluster Computing 20, 3 (2017), 2465-2477. 34

[99] Meidan, Y., Bohadana, M., Mathov, Y., Mirsky, Y., Breitenbacher, D., Shabtai, A., And Elovici, Y. N-baiot: Network-based detection of iot botnet attacks using deep autoencoders. arXiv preprint arXiv:1805.03409 (2018). 38 
[100] Mikolov, T., Chen, K., Corrado, G., and Dean, J. Efficient estimation of word representations in vector space. arXiv preprint arXiv:1301.3781 (2013). 113

[101] Montes, J., Sánchez, A., Memishi, B., Pérez, M. S., And Antoniu, G. Gmone: A complete approach to cloud monitoring. Future Generation Computer Systems 29, 8 (2013), 2026-2040. 42

[102] Morabito, R., And BeiJar, N. Enabling data processing at the network edge through lightweight virtualization technologies. In Sensing, Communication and Networking (SECON Workshops), 2016 IEEE International Conference on (2016), IEEE, pp. 1-6. 22

[103] New relic, May 2018. https://newrelic.com/, 43

[104] Nemman, H. B., Legrand, I. C., Galvez, P., Voicu, R., and Cirstoiu, C. Monalisa: A distributed monitoring service architecture. arXiv preprint cs/0306096 (2003). 41

[105] Ngu, A. H., Gutierrez, M., Metsis, V., Nepal, S., and Sheng, Q. Z. Iot middleware: A survey on issues and enabling technologies. IEEE Internet of Things Journal 4, 1 (2017), 1-20. 17

[106] Pahl, C., Helmer, S., Miori, L., Sanin, J., And Lee, B. A container-based edge cloud paas architecture based on raspberry pi clusters. In Future Internet of Things and Cloud Workshops (FiCloudW), IEEE International Conference on (2016), IEEE, pp. 117-124. 22

[107] Pedregosa, F., Varoquaux, G., Gramfort, A., Michel, V., Thirion, B., Grisel, O., Blondel, M., Prettenhofer, P., Weiss, R., Dubourg, V., Vanderplas, J., Passos, A., Cournapeau, D., Brucher, M., Perrot, M., And Duchesnay, E. Scikit-learn: Machine learning in Python. Journal of Machine Learning Research 12 (2011), 2825-2830. 136

[108] Petridis, P., Gounaris, A., And Torres, J. Spark parameter tuning via trial-and-error. In INNS Conference on Big Data (2016), Springer, pp. 226-237. 37 
[109] Phillips, D. C., And Soltis, J. F. Perspectives on learning. Teachers College Press, 2004. 112

[110] PiaO, J. T., AND YAN, J. A network-aware virtual machine placement and migration approach in cloud computing. In Grid and Cooperative Computing (GCC), 2010 9th International Conference on (2010), IEEE, pp. 87-92. 102

[111] Pongle, P., And Chavan, G. Real time intrusion and wormhole attack detection in internet of things. International Journal of Computer Applications 121, 9 (2015). 38

[112] Povedano-Molina, J., Lopez-Vega, J. M., Lopez-Soler, J. M., Corradi, A., And Foschini, L. Dargos: A highly adaptable and scalable monitoring architecture for multi-tenant clouds. Future Generation Computer Systems 29, 8 (2013), 2041-2056. 42

[113] Prometheus, May 2018. https://prometheus.io/, 43,72

[114] Radojević, B., And ŽAGAR, M. Analysis of issues with load balancing algorithms in hosted (cloud) environments. In MIPRO, 2011 Proceedings of the 34th International Convention (2011), IEEE, pp. 416-420. 102

[115] Rallapalli, S., Ganesan, A., Chintalapudi, K., Padmanabhan, V. N., AND QIU, L. Enabling physical analytics in retail stores using smart glasses. In Proceedings of the 20th Annual International Conference on Mobile Computing and Networking (New York, NY, USA, 2014), MobiCom '14, ACM, pp. 115-126. 19

[116] Raza, S., Wallgren, L., And Voigt, T. Svelte: Real-time intrusion detection in the internet of things. Ad hoc networks 11, 8 (2013), 2661-2674. 38

[117] Rufino, J., Alam, M., Ferreira, J., Rehman, A., And Tsang, K. F. Orchestration of containerized microservices for iiot using docker. In Industrial Technology (ICIT), 2017 IEEE International Conference on (2017), IEEE, pp. 1532-1536. 22 
[118] Russell, S. J., Norvig, P., Canny, J. F., Malik, J. M., And Edwards, D. D. Artificial intelligence: a modern approach, vol. 2. Prentice hall Upper Saddle River, 2003. 92

[119] Sahin, S., CaO, W., Zhang, Q., And Liu, L. Jvm configuration management and its performance impact for big data applications. In Big Data (BigData Congress), 2016 IEEE International Congress on (2016), IEEE, pp. 410-417. 36

[120] Sampath, M., Member, S., Sengupta, R., Lafortune, S., Sinnamohideen, K., And Teneketzis, D. C. Failure diagnosis using discrete-event models. IEEE Trans. Contr. Syst. Tech (1996), 105-124. 35

[121] Sandve, G. K., Nekrutenko, A., Taylor, J., and Hovig, E. Ten simple rules for reproducible computational research. PLoS computational biology 9, 10 (2013), e1003285. 160

[122] Schoenfisch, J., Meilicke, C., von Stülpnagel, J., Ortmann, J., And StuCKenschmidT, H. Root cause analysis in it infrastructures using ontologies and abduction in markov logic networks. Information Systems (2017). 35

[123] Shi, W., Cao, J., Zhang, Q., Li, Y., and Xu, L. Edge computing: Vision and challenges. IEEE Internet of Things Journal 3, 5 (2016), 637-646. 18

[124] Shreenivas, D., Raza, S., And Voigt, T. Intrusion detection in the rplconnected 6lowpan networks. In Proceedings of the 3rd ACM International Workshop on IoT Privacy, Trust, and Security (2017), ACM, pp. 31-38. 38

[125] Shukla, A., Chaturvedi, S., And Simmhan, Y. Riotbench: A real-time iot benchmark for distributed stream processing platforms. CoRR abs/1701.08530 (2017). 27

[126] Shvachko, K., Kuang, H., Radia, S., And Chansler, R. The hadoop distributed file system. In Mass storage systems and technologies (MSST), 2010 IEEE 26th symposium on (2010), Ieee, pp. 1-10. 100

[127] Singh, D., And Reddy, C. K. A survey on platforms for big data analytics. Journal of Big Data 2, 1 (2015), 8. 25 
[128] Solé, M., Muntés-Mulero, V., Rana, A. I., and Estrada, G. Survey on models and techniques for root-cause analysis. arXiv preprint arXiv:1701.08546 (2017). 35

[129] Sorsa, T., And Korvo, H. N. Application of artificial neural networks in process fault diagnosis. Automatica 29, 4 (1993), 843-849. 35

[130] Stojmenovic, I., And Wen, S. The fog computing paradigm: Scenarios and security issues. In Computer Science and Information Systems (FedCSIS), 2014 Federated Conference on (2014), IEEE, pp. 1-8. 17

[131] Taherizadeh, S., Jones, A. C., Taylor, I., Zhao, Z., and Stankovski, V. Monitoring self-adaptive applications within edge computing frameworks: A state-of-the-art review. Journal of Systems and Software 136 (2018), 19-38. 40

[132] Thalheim, J., Rodrigues, A., Akkus, I. E., Bhatotia, P., Chen, R., Viswanath, B., Jiao, L., And Fetzer, C. Sieve: Actionable insights from monitored metrics in microservices. arXiv preprint arXiv:1709.06686 (2017). 35

[133] Toffetti, G., Brunner, S., Blöchlinger, M., Dudouet, F., And EdMONDS, A. An architecture for self-managing microservices. In Proceedings of the 1st International Workshop on Automated Incident Management in Cloud (2015), ACM, pp. 19-24. 34

[134] Tso, F. P., White, D. R., Jouet, S., Singer, J., And Pezaros, D. P. The glasgow raspberry pi cloud: A scale model for cloud computing infrastructures. In Distributed Computing Systems Workshops (ICDCSW), 2013 IEEE 33rd International Conference on (2013), IEEE, pp. 108-112. 17, 71

[135] Uckelmann, D., Harrison, M., and Michahelles, F. An architectural approach towards the future internet of things. In Architecting the internet of things. Springer, 2011, pp. 1-24. 14

[136] Van Renesse, R., Birman, K. P., And Vogels, W. Astrolabe: A robust and scalable technology for distributed system monitoring, management, and data mining. ACM transactions on computer systems (TOCS) 21, 2 (2003), 164-206. 41 
[137] Vaquero, L. M., and Rodero-Merino, L. Finding your way in the fog: Towards a comprehensive definition of fog computing. ACM SIGCOMM Computer Communication Review 44, 5 (2014), 27-32. 19,59

[138] Varshney, P., And Simmhan, Y. Demystifying fog computing: Characterizing architectures, applications and abstractions. In Fog and Edge Computing (ICFEC), 2017 IEEE 1st International Conference on (2017), IEEE, pp. 115-124. 18

[139] Vavilapalli, V. K., Murthy, A. C., Douglas, C., Agarwal, S., Konar, M., Evans, R., Graves, T., Lowe, J., Shah, H., Seth, S., Et Al. Apache hadoop yarn: Yet another resource negotiator. In Proceedings of the 4 th annual Symposium on Cloud Computing (2013), ACM, p. 5. 122

[140] Vilalta, R., Lopez, V., Giorgetti, A., Peng, S., Orsini, V., Velasco, L., Serral-Gracia, R., Morris, D., De Fina, S., Cugini, F., et al. Telcofog: A unified flexible fog and cloud computing architecture for $5 \mathrm{~g}$ networks. IEEE Communications Magazine 55, 8 (2017), 36-43. 56

[141] Vögler, M., Schleicher, J. M., Inzinger, C., And Dustdar, S. A scalable framework for provisioning large-scale iot deployments. ACM Transactions on Internet Technology (TOIT) 16, 2 (2016), 11. 22

[142] Wang, L., Zhan, J., Luo, C., Zhu, Y., Yang, Q., He, Y., GaO, W., Jia, Z., Shi, Y., Zhang, S., Zheng, C., Lu, G., Zhan, K., Li, X., And Qiu, B. Bigdatabench: a big data benchmark suite from internet services. CoRR abs/1401.1406 (2014). 28

[143] Willmott, C. J., And Matsuura, K. Advantages of the mean absolute error (mae) over the root mean square error (rmse) in assessing average model performance. Climate research 30, 1 (2005), 79-82. 137

[144] Wong, W. E., Gao, R., Li, Y., Abreu, R., and Wotawa, F. A survey on software fault localization. IEEE Transactions on Software Engineering PP, 99 (2016), 1-1. 35 
[145] Wu, D., Liu, S., Zhang, L., Terpenny, J., Gao, R. X., Kurfess, T., and Guzzo, J. A. A fog computing-based framework for process monitoring and prognosis in cyber-manufacturing. Journal of Manufacturing Systems 43, Part 1 (2017), $25-34.40$

[146] Wu, Z., And Palmer, M. Verbs semantics and lexical selection. In Proceedings of the 32nd annual meeting on Association for Computational Linguistics (1994), Association for Computational Linguistics, pp. 133-138. 96

[147] Xu, L., Li, M., Zhang, L., Butt, A. R., Wang, Y., And Hu, Z. Z. Memtune: Dynamic memory management for in-memory data analytic platforms. In Parallel and Distributed Processing Symposium, 2016 IEEE International (2016), IEEE, pp. 383-392. 37

[148] Yi, S., Li, C., AND Li, Q. A survey of fog computing: concepts, applications and issues. In Proceedings of the 2015 Workshop on Mobile Big Data (2015), ACM, pp. 37-42. 19

[149] Zaharia, M., Xin, R. S., Wendell, P., Das, T., Armbrust, M., Dave, A., Meng, X., Rosen, J., Venkataraman, S., Franklin, M. J., et Al. Apache spark: a unified engine for big data processing. Communications of the ACM 59, 11 (2016), 56-65. 25, 28

[150] Zanella, A., Bui, N., Castellani, A., Vangelista, L., and Zorzi, M. Internet of things for smart cities. IEEE Internet of Things journal 1, 1 (2014), 22-32. 13

[151] Zarpelão, B. B., Miani, R. S., Kawakani, C. T., and de Alvarenga, S. C. A survey of intrusion detection in internet of things. Journal of Network and Computer Applications 84 (2017), 25-37. 38

[152] Zasadziński, M., Solé, M., Brandon, A., Muntés-Mulero, V., and CarRERA, D. Next stop "noops": Enabling cross-system diagnostics through graphbased composition of logs and metrics. In Cluster Computing (CLUSTER), 2018 IEEE International Conference on (2018), IEEE. 8, 84,112 
[153] Zhang, X., Qian, Z., Zhang, S., Li, Y., Li, X., Wang, X., and Lu, S. Towards reliable (and efficient) job executions in a practical geo-distributed data analytics system. arXiv preprint arXiv:1802.00245 (2018). 25

[154] Zhang, Y., Ren, S., LiU, Y., And Si, S. A big data analytics architecture for cleaner manufacturing and maintenance processes of complex products. Journal of Cleaner Production 142 (2017), 626-641. 18

[155] Zhu, Q., Wang, R., Chen, Q., Liu, Y., And Qin, W. Iot gateway: Bridgingwireless sensor networks into internet of things. In Embedded and Ubiquitous Computing (EUC), 2010 IEEE/IFIP 8th International Conference on (2010), Ieee, pp. 347-352. 15 